T. Funaki

Nagoya Math. J.

Vol. 89 (1983), 129-193

\title{
RANDOM MOTION OF STRINGS AND RELATED STOCHASTIC EVOLUTION EQUATIONS*
}

\author{
TADAHISA FUNAKI
}

\section{§. Introduction}

In this paper, we shall investigate the random motion of an elastic string by using the theory of infinite dimensional stochastic differential equations. The paper consists of three main parts and appendices. In the first part (§2), we shall derive a basic equation which describes the random motion of a string. Several properties of this equation will be investigated in $\S 3,4$ and 5 . In the third part (§6), we shall deal with a stochastic differential equation on a Hilbert space as a generalization of the equation of the string.

For given two functions $a: R^{d} \rightarrow R^{d} \otimes R^{d}$ and $b: R^{d} \rightarrow R^{d}$, let $x_{t}$ be a diffusion process on $R^{d}$ determined by the stochastic differential equation:

$$
d x_{t}=a\left(x_{t}\right) d w_{t}+b\left(x_{t}\right) d t,
$$

where $w_{t}$ is a $d$-dimensional Brownian motion.

We shall introduce in $\S 2$ the following $C\left([0,1], R^{d}\right)$-valued stochastic differential equation (0.2) as an equation that describes a string which moves in $R^{d}$ being interfered with by the process $x_{t}$.

$$
\begin{gathered}
d X_{t}(\sigma)=a\left(X_{t}(\sigma)\right) d B_{t}(\sigma)+b\left(X_{t}(\sigma)\right) d t+\frac{\kappa}{2} \frac{\partial^{2}}{\partial \sigma^{2}} X_{t}(\sigma) d t, \\
\sigma \in[0,1], \quad \kappa>0,
\end{gathered}
$$

where $\sigma$ is a parameter of the string and $B_{t}(\sigma)$ is a cylindrical Brownian motion on $L^{2}\left([0,1], R^{d}\right)$, i.e., $\left(\partial B_{t} / \partial t\right)(\sigma)$ is a $d$-dimensional Gaussian white noise with two parameters $(t, \sigma) \in[0, \infty) \times[0,1]$. The equation $(0.2)$ should have some boundary conditions corresponding to the state at edges of the string.

The idea behind the derivation of the equation (0.2) is to take the scaling limit of a sequence of polygonal approximations. More precisely,

Received August 4, 1981.

*) Supported in part by the SAKKOKAI FOUNDATION. 
we approximate the string by a polygon and set on each corner a particle which moves governed by the stochastic differential equation (0.1) with a suitable scaling. In each step of the approximation, the interaction between neighboring two particles is always taken into account.

The Hamiltonian of an elastic string $X \in C\left([0,1], R^{d}\right)$ with modulus $\kappa$ of elasticity which is located in the potential field $U(x)\left(x \in R^{d}\right)$ is given by

$$
H(X)=\int_{0}^{1} U(X(\sigma)) d \sigma+\frac{\kappa}{2} \int_{0}^{1}\left|\frac{\partial X}{\partial \sigma}(\sigma)\right|^{2} d \sigma .
$$

With this $H(X)$, a physical meaning of the equation (0.2) becomes clear when $a(x)=I_{d}(d \times d$-identity matrix $)$ and

$$
b(x)=-\frac{1}{2} \nabla U(x)=-\frac{1}{2}\left(\frac{\partial U}{\partial x_{1}}, \cdots, \frac{\partial U}{\partial x_{d}}\right) .
$$

In this case, the equation (0.2) is rewritten in the following form:

$$
d X_{t}(\sigma)=d B_{t}(\sigma)-\frac{1}{2} \frac{\delta H}{\delta X(\sigma)}\left(X_{t}\right) d t
$$

where $\delta H / \delta X$ is the functional derivative of $H(X)$. This is the infinitedimensional Einstein-Smoluchowski equation which is given by the limit of the Ornstein-Uhlenbeck theory for the string with large friction, i.e., the limit of $\beta \rightarrow \infty$ in the following equation (0.5) which describes the dynamics of the string with friction intensity $\beta$ forced by white noise (see Appendix III).

$$
\left\{\begin{array}{l}
d X_{t}(\sigma)=V_{t}(\sigma) d t \\
d V_{t}(\sigma)=-\frac{\beta}{2} \frac{\delta H}{\delta X(\sigma)}\left(X_{t}\right) d t-\beta V_{t}(\sigma) d t+\beta d B_{t}(\sigma) .
\end{array}\right.
$$

We shall then study the properties of the equation (0.2) of the string in the following four aspects.

(a) To look for the limit of the solution of the equation (0.2) as the parameter $\kappa$ tends to infinity.

(b) To give a stationary reversible measure of the equation (0.2).

(c) To discuss the motion from some topological view points.

(d) To study the recurrent properties of the random motion of the string.

In $\S 3$, we shall answer to the problem (a). The limit processes are different according as situations of both edges of the string. Roughly 
speaking, our results are the following. In the case where both edges move freely and independently, the string shrinks to one point whose motion is governed by the original stochastic differential equation (0.1); in the case where one end is fixed at a point $A_{0} \in R^{d}$ while the other moves freely, the string contracts to the point $A_{0}$; in the case where both ends are fixed at two points $A_{0}, A_{1} \in R^{d}$, respectively, the string converges to the segment $\overline{A_{0} A_{1}}$. We shall also discuss the deviations from the limit processes.

In $\S 4$, assuming that $a(x)=I_{d}$ and $b(x)=-\nabla U(x) / 2$, namely, considering the equation (0.4), we shall give a stationary reversible measure of this equation explicitly. This measure is the infinite-dimensional analogue to that given by A. N. Kolmogorov [11] in the finite-dimensional case. Using this expression of the stationary measure, in the case where the potential function $U(x)$ diverges on some closed region $E$ in $R^{d}$ and where the both edges are fixed in $D=E^{c}$ (the complement of the region $E$ ), we shall also construct a process that may be thought of as a stationary solution of the equation (0.4). Since we can show that the string never touches the domain $\stackrel{\circ}{E}$ (the interior of $E$ ), this stationary solution induces a probability measure $P$ on the space $C([0, \infty), C([0,1], \bar{D}))$. As a simple answer to the problem (c), we shall give a decomposition of $P$ according as the fundamental group $\pi_{1}(\bar{D})$ of the region $\bar{D}$. By using S. A. Molchanov's results [17], we shall also be able to answer to the problem (a) in case a divergent potential is given.

In $\S 5$, the recurrent properties of two dimensional strings with free edges will be discussed. Assuming that $d=2, \kappa=1, a(x)=I_{2}$ and $b(x)$ $=0$, we will get the following two results.

(i) The solution $X_{t}$ of the equation (0.2) is recurrent as a $C\left([0,1], R^{2}\right)$ valued process.

(ii) The string $X_{t}(\sigma)$ sweeps away all points in $R^{2}$ with probability 1 .

Finally in $\S 6$, we shall discuss, as a generalization of the equation (0.2), the following semi-linear stochastic differential equation (0.6) on a Hilbert space $H$.

$$
d X_{t}=a\left(X_{t}\right) d B_{t}+b\left(X_{t}\right) d t-\kappa A X_{t} d t,
$$

where $a: H \rightarrow \mathscr{L}(H)=\{$ the set of all bounded linear operators on $H\}, b$ : $H \rightarrow H, B_{t}$ is a cylindrical Brownian motion on $H$ and $A$ is a self-adjoint, non-negative operator which has pure point spectrum $\left\{\lambda_{k}\right\}_{k=1}^{\infty} ; 0 \leqq \lambda_{1} \leqq$ 
$\lambda_{2} \leqq \cdots$ such that $\lambda_{k} \sim c k^{1+\delta}(c, \delta>0)$ as $k \rightarrow \infty$. Such an equation was introduced and discussed by D. A. Dawson [6]. With his results in mind, we will give the limit or the scaling limit of the solutions indexed by $\kappa$ of the equation (0.6) by letting $\kappa \rightarrow \infty$.

The author wishes to thank Professor T. Hida for valuable suggestions and kind encouragement in preparing the manuscript.

\section{§1. Preliminaries}

(a) The continuity of sample functions of random fields with $n$ parameters.

Let $C\left(R^{n}\right)$ be a set of all real-valued continuous functions defined on $R^{n}$. A sufficient condition for tightness of a sequence $\left\{X_{N}\right\}_{N=1}^{\infty}$ of random elements of $C\left(R^{n}\right)$ is given by the following proposition.

Proposition 1.1. The sequence $\left\{X_{N}\right\}$ is tight if it satisfies these two conditions:

(i) There exists $\gamma>0$ such that

$$
\sup _{N} E\left[\left|X_{N}(0)\right|^{r}\right]<\infty .
$$

(ii) For each $M<\infty$, there exist $\alpha, \beta$ and $C>0$ such that

$$
\begin{aligned}
& \sup _{N} E\left[\left|X_{N}(x)-X_{N}(y)\right|^{\alpha}\right] \leqq C|x-y|^{n+\beta} \\
& \qquad \text { for all } x, y \in R^{n}:|x|,|y| \leqq M .
\end{aligned}
$$

For the continuity of sample functions of random fields defined on $R^{n}$, the following result is known (see, e.g., Yu. N. Blagovescenskii and M. I. Freidlin [3]).

Proposition 1.2. Suppose that a real-valued separable random field $X(x)$ defined for $x \in R^{n}$ satisfies the following two conditions.

(i) There exists $\gamma>0$ such that

$$
E[|X(0)| r]<\infty .
$$

(ii) For each $M<\infty$, there exist $\alpha, \beta$ and $C>0$ such that

$$
\begin{aligned}
E\left[|X(x)-X(y)|^{\alpha}\right] & \leqq C|x-y|^{n+\beta} \\
& \text { for all } x, y \in R^{n}:|x|,|y| \leqq M .
\end{aligned}
$$

Then, $X(x)$ has a version continuous in $x$. 
(b) A cylindrical Brownian motion on a Hilbert space.

Let $H$ be a separable real Hilbert space with inner product $\langle$,$\rangle and$ norm $\|\cdot\|$. Suppose a complete probability space $(\Omega, \mathscr{F}, P)$ and an increasing family $\left\{\mathscr{F}_{t} ; t \geqq 0\right\}$ of sub- $\sigma$-fields of $\mathscr{F}$ be given. After M. Yor [19], we define a cylindrical Brownian motion on $H$ as follows.

Definition 1.1. A family of random linear functionals $\left\{B_{t} ; t \geqq 0\right\}$ on $H$ is called a cylindrical Brownian motion on $H$ if it satisfies the following conditions:

(i ) $B_{0}(\phi)=0$ and $B_{t}(\phi)$ is $\mathscr{F}_{t}$-adapted for every $\phi \in H$.

(ii) For every $\phi \in H(\phi \neq 0), B_{t}(\phi) /\|\phi\|$ is a one-dimensional Brownian motion.

We often denote $B_{t}(\phi)$ by $\left\langle B_{t}, \phi\right\rangle$ or $\left\langle\phi, B_{t}\right\rangle$. Sometimes, $B_{t}$ is called a Siegel process (R. Holley and D. Stroock [9]) or a standard Wiener $\mathscr{S}^{\prime}$ process in case $H=L^{2}\left(R^{n}\right)$ (K. Itô [10]). When $H=L^{2}(G), G$ being a domain in $R^{n}$, we shall denote $\left\langle B_{t}, \phi\right\rangle$ by

$$
\int_{G} B_{t}(\sigma) \phi(\sigma) d \sigma, \quad \phi \in H
$$

(c) Stochastic integrals with respect to the cylindrical Brownian motion.

For an $H$-valued $\mathscr{F}_{t}$-adapted measurable function $f(t, \omega) \quad((t, \omega) \epsilon$ $[0, T] \times \Omega)$ such that

$$
E\left[\int_{0}^{T}\|f(t, \omega)\|^{2} d t\right]<\infty
$$

we define a real valued stochastic integral $\int_{0}^{T}\left\langle f(t), d B_{t}\right\rangle$ by

$$
\sum_{n=1}^{\infty} \int_{0}^{T}\left\langle f(t), \phi_{n}\right\rangle d B_{t}\left(\phi_{n}\right)
$$

where $\left\{\phi_{n}\right\}$ is a complete orthonormal system in $H$. With this notation we have

$$
E\left[\left|\int_{0}^{T}\left\langle f(t), d B_{t}\right\rangle\right|^{2}\right]=E\left[\int_{0}^{T}\|f(t)\|^{2} d t\right]
$$

If $H=L^{2}(G)$, then we shall denote the stochastic integral $\int_{0}^{T}\left\langle f(t), d B_{t}\right\rangle$ by

$$
\int_{0}^{T} \int_{G} f(t, \sigma) d B_{t}(\sigma) d \sigma
$$


Let $\mathscr{L}_{2}(H)$ be the set of all Hilbert-Schmidt operators on $H$, and denote the Hilbert-Schmidt norm by $\|\cdot\|_{2}$. For an $\mathscr{L}_{2}(H)$-valued $\mathscr{F}_{t}$-adapted measurable function $F(t, \omega)$, we define an $H$-valued stochastic integral $\int_{0}^{T} F(t) d B_{t}$ by the following equality:

$$
\left\langle\int_{0}^{T} F(t) d B_{t}, \phi\right\rangle=\int_{0}^{T}\left\langle F^{*}(t) \phi, d B_{t}\right\rangle \quad \text { for every } \phi \in H,
$$

where $F^{*}$ is the adjoint operator of $F$. Then we have

$$
E\left[\left\|\int_{0}^{T} F(t) d B_{t}\right\|^{2}\right]=E\left[\int_{0}^{T}\|F(t)\|_{2}^{2} d t\right] .
$$

By analogy with the finite dimensional case (I.I. Gihman and A. V. Skorohod [8]), the following estimates hold for the stochastic integrals with respect to the cylindrical Brownian motion.

Proposition 1.3. (i ) For $p=1,2, \cdots$, there exists a positive constant $C=C(p)$ such that

$$
E\left[\left(\int_{0}^{T}\left\langle f(t), d B_{t}\right\rangle\right)^{2 p}\right] \leqq C\left\{\int_{0}^{T} E\left[\|f(t)\|^{2 p}\right]^{1 / p} d t\right\}^{p}
$$

and

$$
E\left[\left(\int_{0}^{T}\left\langle f(t), d B_{t}\right\rangle\right)^{2 p}\right] \leqq C T^{p-1} \int_{0}^{T} E\left[\|f(t)\|^{2 p}\right] d t
$$

for every $H$-valued $\mathscr{F}_{t}$-adapted measurable function $f(t, \omega)$ which satisfies

$$
\int_{0}^{T} E\left[\|f(t)\|^{2 p}\right] d t<\infty .
$$

(ii) For each $\mathscr{L}_{2}(H)$-valued $\mathscr{F}_{t}$-adapted measurable function $F(t, \omega)$ which satisfies

$$
\int_{0}^{T} E\left[\|F(t)\|_{2}^{2 p}\right] d t<\infty
$$

the following estimates hold.

$$
\begin{aligned}
& E\left[\left\|\int_{0}^{T} F(t) d B_{t}\right\|^{2 p}\right] \leqq C\left\{\int_{0}^{T} E\left[\|F(t)\|_{2}^{2 p}\right]^{1 / p} d t\right\}^{p} \\
& E\left[\left\|\int_{0}^{T} F(t) d B_{t}\right\|^{2 p}\right] \leqq C T^{p-1} \int_{0}^{T} E\left[\|F(t)\|_{2}^{2 p}\right] d t .
\end{aligned}
$$

Proof. We show only the estimates in (i), since those in (ii) can be 
shown similarly. Using Itô's formula in the infinite dimensional case (M. Yor [19]), we have

$$
\begin{aligned}
& E\left[\left(\int_{0}^{t}\left\langle f(s), d B_{s}\right\rangle\right)^{2 p}\right]=p(2 p-1) \int_{0}^{t} d s E\left[\left(\int_{0}^{s}\left\langle f(u), d B_{u}\right\rangle\right)^{2 p-2}\|f(s)\|^{2}\right] \\
& \text { for } t<T \text {. }
\end{aligned}
$$

By Hölder's inequality,

$$
\begin{aligned}
& E\left[\left(\int_{0}^{t}\left\langle f(s), d B_{s}\right\rangle\right)^{2 p}\right] \\
& \quad \leqq p(2 p-1) \int_{0}^{t} d s E\left[\left(\int_{0}^{s}\left\langle f(u), d B_{u}\right\rangle\right)^{2 p}\right]^{(p-1) / p} E\left[\|f(s)\|^{2 p}\right]^{1 / p} \\
& \quad \leqq p(2 p-1) E\left[\left(\int_{0}^{t}\left\langle f(s), d B_{s}\right\rangle\right)^{2 p}\right]^{(p-1) / p} \int_{0}^{t} E\left[\|f(s)\|^{2 p}\right]^{1 / p} d s .
\end{aligned}
$$

We, therefore, have (1.3) with $C=\{p(2 p-1)\}^{p}$. The estimate (1.4) can be derived from (1.3) by using Hölder's inequality.

(d) Stochastic differential equations on a Hilbert space.

Consider the following non-linear stochastic evolution equation on $H$.

$$
\left\{\begin{array}{l}
d X_{t}=a\left(t, X_{t}\right) d B_{t}+b\left(t, X_{t}\right) d t-A X_{t} d t, \quad t \in[0, T] \\
X_{0} \in H,
\end{array}\right.
$$

where $B_{t}$ is the cylindrical Brownian motion on $H$. We assume that $A$, $a$ and $b$ satisfy the following three conditions.

(A.1) $A$ is a non-negative, self-adjoint operator on $H$ and has pure point spectrum $\left\{\lambda_{k}\right\}_{k=1}^{\infty} ; 0 \leqq \lambda_{1} \leqq \lambda_{2} \leqq \cdots$, such that $\lambda_{k} \sim c k^{1+\delta}(c, \delta>0)$ as $k \rightarrow \infty$.

(A.2) $a(\cdot, \cdot)$ is a mapping from $[0, T] \times H$ into the set $\mathscr{L}(H)$ of all bounded linear operators on $H$ and satisfies the following two conditions.

(i) $a^{*}(\cdot, \cdot) \phi_{k}$ is a continuous mapping from $[0, T] \times H$ into $H$ for each $k=1,2, \cdots$,

(ii) $\left\|a^{*}(t, X) \phi_{k}\right\| \leqq K(1+\|X\|)$, $\left\|\left(a^{*}\left(t, X_{1}\right)-a^{*}\left(t, X_{2}\right)\right) \phi_{k}\right\| \leqq K\left\|X_{1}-X_{2}\right\|, \quad K>0$, for every $X, X_{1}, X_{2} \in H, t \in[0, T]$ and $k=1,2, \cdots$, where $a^{*}(t, X)$ is the adjoint operator of $a(t, X)$ and $\phi_{k}$ is the normalized eigenvector of $A$ corresponding to the eigenvalue $\lambda_{k}$.

(A.3) $b(\cdot, \cdot)$ is a continuous mapping from $[0, T] \times H$ into $H$ and satisfies 


$$
\begin{aligned}
& \left\|b\left(t, X_{1}\right)-b\left(t, X_{2}\right)\right\| \leqq K\left\|X_{1}-X_{2}\right\|, \quad K>0, \\
& \quad \text { for every } X_{1}, X_{2} \in H \text { and } t \in[0, T] .
\end{aligned}
$$

An $H$-valued $\mathscr{F}_{t}$-adapted process $X_{t}$ is called a solution of the equation (1.7), if it satisfies the following integral equation:

$$
X_{t}=U(t) X_{0}+\int_{0}^{t} U(t-s) a\left(s, X_{s}\right) d B_{s}+\int_{0}^{t} U(t-s) b\left(s, X_{s}\right) d s,
$$

where $\{U(t), t \geqq 0\}$ is a semi-group on $H$ generated by $-A$. The following theorem was shown by D. A. Dawson [6].

TheOREM 1.1. There exists a unique solution $X_{t}$ of the equation (1.7) such that $\sup _{t \in[0, T]} E\left[\left\|X_{t}\right\|^{2}\right]<\infty$ for each $T<\infty$ and $X_{t} \in C([0, \infty), H)$ (a.s.).

In Appendix I, we shall discuss the case of $H=L^{2}\left(G, R^{d}\right)$ with a bounded domain $G$ in $R^{n}$. In this case, the solution $X_{t}(\cdot)$ of the equation (1.7) determines an $R^{d}$-valued random field defined for $(t, \sigma) \in[0, \infty) \times G$. We shall study the joint continuity of sample functions of the $X_{t}(\sigma)$ in $(t, \sigma)$.

\section{§2. The stochastic differential equations of randomly fluctuating strings}

Let $x_{t}$ be a diffusion process on $R^{d}$ determined by the following stochastic differential equation:

$$
d x_{t}=a\left(x_{t}\right) d w_{t}+b\left(x_{t}\right) d t, \quad t>0,
$$

where $w_{t}$ is a $d$-dimensional Brownian motion, and where $a$ and $b$ are $R^{d} \otimes R^{d}$ - and $R^{d}$-valued Lipschitz continuous functions on $R^{d}$, respectively. In addition, $a$ is assumed to be bounded.

In this section, we derive the stochastic equation (0.2) of an elastic string which moves in $R^{d}$ being interfered with by the process $x_{t}$. The state space of continuous strings, denote them by $X(\sigma), \sigma \in[0,1]$, is a function space $\mathscr{C}=C\left([0,1], R^{d}\right)$ topologized by the uniform-norm.

The state space is often specified to a subset of $\mathscr{C}$, so that we are led to consider the following three cases.

( I ) Both ends of the string move freely.

(II) One end of the string is fixed at a point $A_{0} \in R^{d}$ while the other end moves freely.

(III) Both ends are fixed at points $A_{0}, A_{1} \in R^{d}$, respectively. 
The initial state $X_{0} \in \mathscr{C}$ of the string is assumed to satisfy the following conditions in the cases (II) and (III).

$$
\left\{\begin{array}{l}
X_{0}(0)=A_{0}, \quad \text { in the case (II). } \\
X_{0}(0)=A_{0}, \quad X_{0}(1)=A_{1}, \quad \text { in the case (III) . }
\end{array}\right.
$$

In the case (I), nothing is assumed for $X_{0}$.

We are now ready to derive the equation by the polygonal approximation. Let $\left\{w_{t}(k)\right\}_{k=1}^{\infty}$ be an infinite system of independent $d$-dimensional Brownian motions. We determine, for a positive integer $N$, an $N \times$ $d$-dimensional diffusion process $\left\{X_{t}^{\left(N^{\prime}\right)}(k / N)\right\}_{k=1}^{N}$ by the stochastic differential equation:

$$
\left\{\begin{aligned}
d X_{t}^{(N)}\left(\frac{k}{N}\right)=\sqrt{N} a\left(X_{t}^{(N)}\left(\frac{k}{N}\right)\right) d w_{t}(k) & +b\left(X_{t}^{(N)}\left(\frac{k}{N}\right)\right) d t \\
& +\frac{\kappa}{2} \Delta^{(N)} X_{t}^{(N)}\left(\frac{k}{N}\right) d t, \\
1 \leqq k \leqq N, \quad \kappa>0, \quad t>0, &
\end{aligned}\right.
$$

where

$$
\Delta^{(N)} X_{t}^{(N)}\left(\frac{k}{N}\right)=\left\{\Delta^{(N)}\left(X_{t}^{(N)}\left(\frac{k}{N}\right)\right)^{(i)}\right\}_{i=1}^{d}, \quad X_{t}^{(N)}\left(\frac{k}{N}\right)=\left\{\left(X_{t}^{(N)}\left(\frac{k}{N}\right)\right)^{(i)}\right\}_{i=1}^{d}
$$

and where

$$
\begin{aligned}
& \Delta^{(N)}\left(X_{t}^{(N)}\left(\frac{k}{N}\right)\right)^{(i)} \\
& \quad=N^{2}\left\{\left(X_{t}^{(N)}\left(\frac{k+1}{N}\right)\right)^{(i)}-2\left(X_{t}^{(N)}\left(\frac{k}{N}\right)\right)^{(i)}+\left(X_{t}^{(N)}\left(\frac{k-1}{N}\right)\right)^{(i)}\right\} .
\end{aligned}
$$

The following boundary conditions are given according as cases (I)-(III).

$$
\begin{cases}X_{t}^{(N)}(0)=X_{t}^{(N)}\left(\frac{1}{N}\right), \quad X_{t}^{(N)}\left(\frac{N+1}{N}\right)=X_{t}^{(N)}(1), & t>0 \\ & \text { in the case (I) } \\ X_{t}^{(N)}(0)=A_{0}, \quad X_{t}^{(N)}\left(\frac{N+1}{N}\right)=X_{t}^{(N)}(1), \quad t>0, & \text { in the case (II). } \\ X_{t}^{(N)}(0)=A_{0}, \quad X_{t}^{(N)}\left(\frac{N+1}{N}\right)=A_{1}, \quad t>0, & \text { in the case (III). }\end{cases}
$$


The physical meaning of the equation (2.3) is as follows. The movement of the $N$ particles $\left\{X_{t}^{(N)}(k / N)\right\}_{k=1}^{N}$ on the string is governed by a system of stochastic differential equations which have the diffusion coefficient $\sqrt{N} a(x)$ and the drift coefficient $b(x)$. The random fluctuation forces are understood to be mutually independent. Two neighboring particles attract each other by the force $\kappa N^{2}\left|X_{t}^{(N)}((k+1) / N)-X_{t}^{(N)}(k / N)\right| / 2(0 \leqq k \leqq N)$. The constant $\kappa$ is the modulus of elasticity of the string.

We define $X_{t}^{(N)}(\sigma), \sigma \in[0,1]$, by

$$
\begin{gathered}
X_{t}^{(N)}(\sigma)=(N \sigma-k+1) X_{t}^{(N)}\left(\frac{k}{N}\right)+(k-N \sigma) X_{t}^{(N)}\left(\frac{k-1}{N}\right), \\
\sigma \in\left[\frac{k-1}{N}, \frac{k}{N}\right], \quad 1 \leqq k \leqq N .
\end{gathered}
$$

A probability measure $P^{(N)}$ is introduced on $C([0, \infty), \mathscr{C})$ by $X_{t}^{(N)}$, since the process lives in $C([0, \infty), \mathscr{C})$.

We now pause to consider the stochastic partial differential equation of semi-linear type:

$$
\begin{gathered}
d X_{t}(\sigma)=a\left(X_{t}(\sigma)\right) d B_{t}(\sigma)+b\left(X_{t}(\sigma)\right) d t+\frac{\kappa}{2} \Delta X_{t}(\sigma) d t, \\
\sigma \in[0,1], \quad t>0,
\end{gathered}
$$

with the boundary conditions,

$$
\left\{\begin{array}{l}
\frac{\partial}{\partial \sigma} X_{t}(0)=\frac{\partial}{\partial \sigma} X_{t}(1)=0, \quad \text { in the case }(\mathrm{I}), \\
X_{t}(0)=A_{0}, \quad \frac{\partial}{\partial \sigma} X_{t}(1)=0, \quad \text { in the case (II), } \\
X_{t}(0)=A_{0}, \quad X_{t}(1)=A_{1}, \quad \text { in the case (III), }
\end{array}\right.
$$

where $B_{t}$ is a cylindrical Brownian motion on $L^{2}\left([0,1], R^{d}\right)$, and where $\Delta X_{t}(\sigma)=\left\{\Delta\left(X_{t}(\sigma)\right)^{(i)}\right\}_{i=1}^{d}, X_{t}(\sigma)=\left\{\left(X_{t}(\sigma)\right)^{(i)}\right\}_{i=1}^{d}$ and $\Delta\left(X_{t}(\sigma)\right)^{(i)}=\left(\partial^{2} / \partial \sigma^{2}\right)\left(X_{t}(\sigma)\right)^{(i)}$.

By a solution of the equation (2.5) with the boundary condition (2.6) and the initial state $X_{0} \in \mathscr{C}$, we mean a solution of the stochastic integral equation:

$$
\begin{gathered}
X_{t}(\sigma)=X_{t, 1}(\sigma)+\int_{0}^{t} \int_{0}^{1} p(t-s, \sigma, \tau) a\left(X_{s}(\tau)\right) d B_{s}(\tau) d \tau \\
+\int_{0}^{t} \int_{0}^{1} p(t-s, \sigma, \tau) b\left(X_{s}(\tau)\right) d s d \tau
\end{gathered}
$$

where $X_{t, 1}(\sigma)$ is the solution of the equation: 


$$
\frac{\partial}{\partial t} X_{t, 1}(\sigma)=\frac{\kappa}{2} \Delta X_{t, 1}(\sigma)
$$

with the boundary condition (2.6) and with the initial state $X_{0}(\sigma)$, and where $p(t, \sigma, \tau)$ is the fundamental solution of $(\kappa / 2) \Delta$ with the boundary conditions:

$$
\begin{cases}\frac{\partial p}{\partial \sigma}(t, 0, \tau)=\frac{\partial p}{\partial \sigma}(t, 1, \tau)=0, & \text { in the case (I), } \\ p(t, 0, \tau)=\frac{\partial p}{\partial \sigma}(t, 1, \tau)=0, & \text { in the case (II) } \\ p(t, 0, \tau)=p(t, 1, \tau)=0, & \text { in the case (III). }\end{cases}
$$

We denote by $X_{t, 2}(\sigma)$ and $X_{t, 3}(\sigma)$, the second term and the third term of the right hand side of the equation (2.7), respectively. When $\kappa=1$, the fundamental solution will be denoted by $q(t, \sigma, \tau)$ instead of $p(t, \sigma, \tau)$.

By the argument in Appendix I, the equation (2.5) has a unique solution $X_{t}$ living in $C([0, \infty), \mathscr{C})$ (a.s.). We denote by $P$ the probability measure on $C([0, \infty), \mathscr{C})$ induced by $X_{t}$. In the rest of this section, assuming $\kappa=1$ for simplicity, we shall show that $X_{t}^{(N)}$ converges to the $X_{t}$ as $N \rightarrow \infty$ in the following sence.

Theorem 2.1. As probability measures on $C([0, \infty), \mathscr{C}), P^{(N)}$ tends weakly to $P$ as $N \rightarrow \infty$.

To prove this theorem, first, we rewrite the equation (2.3) in an integral form. Let $q^{(N)}(t, k / N, l / N)(t \geqq 0,0 \leqq k, l \leqq N+1)$ be the fundamental solution of $(\kappa / 2) \Delta^{(N)}$, i.e., a solution of the equation:

$$
\left\{\begin{array}{l}
\frac{\partial}{\partial t} q^{(N)}\left(t, \frac{k}{N}, \frac{\ell}{N}\right)=\frac{1}{2} \Delta_{(k)}^{(N)} q^{(N)}\left(t, \frac{k}{N}, \frac{\ell}{N}\right) \\
\quad t>0, \quad 1 \leqq k, \ell \leqq N \\
q^{(N)}\left(0, \frac{k}{N}, \frac{\ell}{N}\right)=N \delta_{k, \ell}
\end{array}\right.
$$

with the boundary conditions,

$$
\left\{\begin{aligned}
q^{(N)}\left(t, 0, \frac{\ell}{N}\right)=q^{(N)}\left(t, \frac{1}{N}, \frac{\ell}{N}\right), & q^{(N)}\left(t, \frac{N+1}{N}, \frac{\ell}{N}\right)=q^{(N)}\left(t, 1, \frac{\ell}{N}\right), \\
t & >0,1 \leqq \ell \leqq N, \text { in the case (I), }
\end{aligned}\right.
$$




$$
\mid \begin{aligned}
& q^{(N)}\left(t, 0, \frac{\ell}{N}\right)=0, q^{(N)}\left(t, \frac{N+1}{N}, \frac{\ell}{N}\right)=q^{(N)}\left(t, 1, \frac{\ell}{N}\right), \\
& t>0,1 \leqq \ell \leqq N, \text { in the case (II) } \\
& q^{(N)}\left(t, 0, \frac{\ell}{N}\right)=q^{(N)}\left(t, \frac{N+1}{N}, \frac{\ell}{N}\right)=0, \\
& t>0,1 \leqq \ell \leqq N,
\end{aligned}
$$

Noting that $\left\{<B_{t}(\sigma), \sqrt{N} \chi_{[(k-1) / N, k / N)}(\sigma)>\right\}_{k=1}^{N}$ is a system of $N$ independent $d$-dimensional Brownian motions, the process $X_{t}^{(N)}$ has the same distribution as the solution of the integral equation:

$$
\begin{aligned}
X_{t}^{(N)}(\sigma)= & X_{t, 1}^{(N)}(\sigma)+\int_{0}^{t} \int_{0}^{1} q^{(N)}(t-s, \sigma,[\tau]) a\left(X_{s}^{(N)}([\tau])\right) d B_{s}(\tau) d \tau \\
& +\int_{0}^{t} \int_{0}^{1} q^{(N)}(t-s, \sigma,[\tau]) b\left(X_{s}^{(N)}([\tau])\right) d s d \tau
\end{aligned}
$$

where $[\sigma] \equiv[\sigma]^{(N)}=k / N$,

$$
\begin{gathered}
q^{(N)}\left(t, \sigma, \frac{\ell}{N}\right)=(N \sigma-k+1) q^{(N)}\left(t, \frac{k}{N}, \frac{\ell}{N}\right) \\
+(k-N \sigma) q^{(N)}\left(t, \frac{k-1}{N}, \frac{\ell}{N}\right), \\
X_{t, 1}^{(N)}(\sigma)=(N \sigma-k+1) X_{t, 1}^{(N)}\left(\frac{k}{N}\right)+(k-N \sigma) X_{t, 1}^{(N)}\left(\frac{k-1}{N}\right), \\
\text { for } \sigma \in\left(\frac{k-1}{N}, \frac{k}{N}\right], \quad 1 \leqq k \leqq N,
\end{gathered}
$$

and where $\left\{X_{t, 1}^{(N)}(k / N)\right\}_{k=0}^{N}$ is a solution of the following equation (2.13).

$$
\left\{\begin{array}{l}
\frac{\partial}{\partial t} X_{t, 1}^{(N)}\left(\frac{k}{N}\right)=\frac{1}{2} \Delta^{(N)} X_{t, 1}^{(N)}\left(\frac{k}{N}\right) \\
X_{0,1}^{(N)}\left(\frac{k}{N}\right)=X_{0}\left(\frac{k}{N}\right)
\end{array}\right.
$$

with the boundary condition (2.4). We shall denote by $X_{t, 2}^{(N)}(\sigma)$ and $X_{t, 3}^{(N)}(\sigma)$, the second term and the third term of the right hand side of the equation (2.12), respectively.

For $p>0$ and an $R^{d}$-valued random variable $X(t, \sigma)$ with a parameter $(t, \sigma) \in[0, T] \times[0,1]$, we set

$$
\|X\|_{T, p}=\sup _{(t, \sigma) \in[0, T] \times[0,1]} E\left[|X(t, \sigma)|^{p}\right]^{1 / p} .
$$

When $p_{R}=2$, we denote $\|X\|_{T, 2}$ simply by $\|X\|_{T}$. 
Using the estimates on $q^{(N)}$ and $q$ which are summarized in Appendix II, we have the following uniform estimate on the processes $\left\{X_{t}^{(N)}\right\}_{N=1}^{\infty}$.

LEMma 2.1. For each $p>0$ and $T<\infty$, we have

$$
\sup _{N \in N}\left\|X^{(N)}\right\|_{T, p}<\infty, \quad \text { where } N=\{1,2, \cdots\} \text {. }
$$

Proof. Since

$$
X_{t, 1}^{(N)}(\sigma)=\int_{0}^{1}\left\{X_{0}([\tau])-\tilde{X}^{(N)}([\tau])\right\} q^{(N)}(t, \sigma,[\tau]) d \tau+\tilde{X}^{(N)}(\sigma),
$$

where

$$
\tilde{X}^{(N)}(\sigma)=\left\{\begin{array}{cl}
0 & , \\
A_{0} & \text { in the case (I), } \\
\frac{1}{N+1}\left\{(N+1-N \sigma) A_{0}+N \sigma A_{1}\right\}, & \text { in the case (II), }
\end{array}\right.
$$

we can first show

$$
\sup _{N \in N} \sup _{(t, \sigma) \in[0, T] \times[0,1]}\left|X_{t, 1}^{(N)}(\sigma)\right|=C_{1}<\infty .
$$

Since the function $a$ is bounded, Proposition 1.3 and the estimate (AII.5) in Appendix II prove

$$
\begin{aligned}
& E\left[\left|X_{t, 2}^{(N)}\left(\frac{k}{N}\right)\right|^{2 p}\right] \\
& \leqq C_{2}\left\{\int_{0}^{t} d s E\left[\left(\int_{0}^{1}\left|a\left(X_{s}([\tau])\right)\right|^{2}\left(q^{(N)}\left(t-s, \frac{k}{N},[\tau]\right)\right)^{2} d \tau\right)^{p}\right]^{1 / p}\right\}^{p} \\
& \leqq C_{3}\left\{\int_{0}^{t} q^{(N)}\left(2(t-s), \frac{k}{N}, \frac{k}{N}\right) d s\right\}^{p}=C_{4}<\infty, \quad \text { for } p \in N .
\end{aligned}
$$

In the above expression, $|a|$ stands for the norm of the matrix $a$, i.e., $|a|^{2}=\sum_{i, j} a_{i j}^{2}$ for $a=\left(a_{i j}\right)$. Noting that the Lipschitz continuity of the function $b$ implies the estimate:

$$
|b(x)| \leqq C(1+|x|)
$$

we have

$$
\begin{aligned}
& E\left[\left|X_{t, 3}^{(N)}\left(\frac{k}{N}\right)\right|^{2 p}\right] \\
& \quad \leqq E\left[\left(\int_{0}^{t} d s \int_{0}^{1}\left|b\left(X_{s}^{(N)}([\tau])\right)\right|^{2} d \tau\right)^{p}\right]\left\{\int_{0}^{t} d s \int_{0}^{1}\left(q^{(N)}\left(t-s, \frac{k}{N},[\tau]\right)\right)^{2} d \tau\right\}^{p} \\
& \quad \leqq C_{5} \int_{0}^{t}\left\{1+\sup _{1 \leqq k \leqq N} E\left[\left|X_{s}^{(N)}\left(\frac{k}{N}\right)\right|^{2 p}\right]\right\} d s .
\end{aligned}
$$


By the estimates (2.16)-(2.18), we have

$$
\sup _{1 \leqq k \leqq N} E\left[\left|X_{t}^{(N)}\left(\frac{k}{N}\right)\right|^{2 p}\right] \leqq C_{6} \int_{0}^{t}\left\{1+\sup _{1 \leqq k \leqq N} E\left[\left|X_{s}^{(N)}\left(\frac{k}{N}\right)\right|^{2 p}\right]\right\} d s,
$$

where a positive constant $C_{6}$ is independent of $N$. We obtain the desired estimate (2.14) by the help of Gronwall's lemma for each positive even number $p$. We can prove the estimate (2.14) for general $p>0$ by using the Hölder's inequality.

(Q.E.D.)

To show the tightness of the sequence $\left\{X_{t}^{(N)}\right\}_{N=1}^{\infty}$, we need the following lemma.

LEMMA 2.2. For each $i=2,3, T<\infty$ and $p \in N$, there exists $C=$ $C(T, p)>0$ such that

$$
E\left[\left|X_{t_{1}, i}^{(N)}\left(\sigma_{1}\right)-X_{t_{2}, i}^{(N)}\left(\sigma_{2}\right)\right|^{2 p}\right] \leqq C\left\{\left|t_{1}-t_{2}\right|^{p / 2}+\left|\sigma_{1}-\sigma_{2}\right|^{p / 2}\right\},
$$

for every $t_{1}, t_{2} \in[0, T], \sigma_{1}, \sigma_{2} \in[0,1], N \in N$

Proof. For $0 \leqq t_{1} \leqq t_{2} \leqq T$ and $\sigma_{1}, \sigma_{2} \in[0,1]$ such that $\sigma_{1}=\left[\sigma_{1}\right], \sigma_{2}=\left[\sigma_{2}\right]$, Proposition 1.3 and estimates (AII.5-6) imply

$$
\begin{aligned}
& E\left[\left|X_{t_{1}, 2}^{(N)}\left(\sigma_{1}\right)-X_{t_{2}, 2}^{(N)}\left(\sigma_{2}\right)\right|^{2 p}\right] \\
& \leqq C_{1}\left\{\int _ { 0 } ^ { t _ { 2 } } d s E \left[\left(\int_{0}^{1} \mid a\left(X_{s}^{(N)}([\tau])\right)\left(q^{(N)}\left(t_{1}-s, \sigma_{1},[\tau]\right) \chi_{\left\{s \leqq t_{1}\right\}}\right.\right.\right.\right. \\
& \left.\left.\left.\left.-q^{(N)}\left(t_{2}-s, \sigma_{2},[\tau]\right)\right)\left.\right|^{2} d \tau\right)^{p}\right]^{1 / p}\right\}^{p} \\
& \leqq C_{2}\left\{\int_{0}^{t_{2}} d s \int_{0}^{1}\left(q^{(N)}\left(t_{1}-s, \sigma_{1},[\tau]\right) \chi_{\left\{s \leqq t_{1}\right\}}-q^{(N)}\left(t_{2}-s, \sigma_{2},[\tau]\right)\right)^{2} d \tau\right\}^{p} \\
& =C_{2}\left\{\int_{0}^{t_{1}} q^{(N)}\left(2 s, \sigma_{1}, \sigma_{1}\right) d s+\int_{0}^{t_{2}} q^{(N)}\left(2 s, \sigma_{2}, \sigma_{2}\right) d s\right. \\
& \left.-2 \int_{\left(t_{2}-t_{1}\right) / 2}^{\left(t_{1}+t_{2}\right) / 2} q^{(N)}\left(2 s, \sigma_{1}, \sigma_{2}\right) d s\right\}^{p} \\
& \leqq C_{3}\left\{\left|t_{1}-t_{2}\right|^{1 / 2}+\left|\sigma_{1}-\sigma_{2}\right|^{1 / 2}\right\}^{p} \\
& \leqq C_{4}\left\{\left|t_{1}-t_{2}\right|^{p / 2}+\left|\sigma_{1}-\sigma_{2}\right|^{p / 2}\right\} \text {. }
\end{aligned}
$$

For every $0 \leqq \sigma_{1} \leqq \sigma_{2} \leqq 1$, we have

$$
\begin{aligned}
E\left[\left|X_{t_{1}, 2}^{(N)}\left(\sigma_{1}\right)-X_{t_{2}, 2}^{(N)}\left(\sigma_{2}\right)\right|^{2 p}\right] & \\
\leqq & C_{5}\left\{E\left[\left|X_{t_{1}, 2}^{(N)}\left(\sigma_{1}\right)-X_{t_{1}, 2}^{(N)}\left(\left[\sigma_{1}\right]\right)\right|^{2 p}\right]\right. \\
& \quad+E\left[\mid X_{t_{1}, 2}^{(N)}\left(\left[\sigma_{1}\right]\right)-X_{t_{2}, 2}^{(N)}\left(\left[\sigma_{2}\right]-\frac{1}{N}\right)^{2 p}\right]
\end{aligned}
$$




$$
\begin{aligned}
& \left.+E\left[\left|X_{t_{2}, 2}^{(N)}\left(\left[\sigma_{2}\right]-\frac{1}{N}\right)-X_{t_{2}, 2}^{(N)}\left(\sigma_{2}\right)\right|^{2 p}\right]\right\} \\
= & C_{5}\left\{N^{2 p}\left(\left[\sigma_{1}\right]-\sigma_{1}\right)^{2 p} E\left[\left|X_{t_{1}, 2}^{(N)}\left(\left[\sigma_{1}\right]-\frac{1}{N}\right)-X_{t_{1}, 2}^{(N)}\left(\left[\sigma_{1}\right]\right)\right|^{2 p}\right]\right. \\
& +E\left[\left|X_{t_{1}, 2}^{(N)}\left(\left[\sigma_{1}\right]\right)-X_{t_{2}, 2}^{(N)}\left(\left[\sigma_{2}\right]-\frac{1}{N}\right)\right|^{2 p}\right] \\
& \left.+N^{2 p}\left(\sigma_{2}-\left[\sigma_{2}\right]+\frac{1}{N}\right)^{2 p} E\left[\left|X_{t_{2}, 2}^{(N)}\left(\left[\sigma_{2}\right]-\frac{1}{N}\right)-X_{t_{2}, 2}^{(N)}\left(\left[\sigma_{2}\right]\right)\right|^{2 p}\right]\right\} \\
\leqq & C_{5} C_{4}\left\{\left|\left[\sigma_{1}\right]-\sigma_{1}\right|^{p / 2}+\left|t_{1}-t_{2}\right|^{p / 2}+\left|\left[\sigma_{2}\right]-\frac{1}{N}-\left[\sigma_{1}\right]\right|^{p / 2}\right. \\
& \left.+\left|\sigma_{2}-\left[\sigma_{2}\right]+\frac{1}{N}\right|^{p / 2}\right\} \\
\leqq & C_{5} C_{4}\left\{\left|t_{1}-t_{2}\right|^{p / 2}+\left|\sigma_{1}-\sigma_{2}\right|^{p / 2}\right\} .
\end{aligned}
$$

This proves the estimate (2.19) for $i=2$. The estimate (2.19) for $i=3$ can be proved, in a similar way to the case of $i=2$, with the help of Lemma 2.1 .

(Q.E.D.)

The solution $X_{t}^{(N)}$ of the equation (2.12) converges to the solution $X_{t}$ of the equation (2.7) as $N \rightarrow \infty$ in the following sense.

Proposition 2.1. We have

$$
\lim _{N \rightarrow \infty}\left\|X^{(N)}-X\right\|_{T}=0 \quad \text { for each } T<\infty .
$$

Proof. By (2.15) and (AII.4),

$$
\lim _{N \rightarrow \infty} \sup _{(t, \sigma) \in[0, T] \times[0,1]}\left|X_{t, 1}^{(N)}([\sigma])-X_{t, 1}(\sigma)\right|=0 .
$$

By the Lipschitz continuity and the boundedness of the function $a$,

$$
\begin{aligned}
& E\left[\left|X_{t, 2}^{(N)}([\sigma])-X_{t, 2}(\sigma)\right|^{2}\right] \\
& \leqq C_{1} \int_{0}^{t} d s \int_{0}^{1} E\left[\mid q^{(N)}(t-s,[\sigma],[\tau]) a\left(X_{s}^{(N)}([\tau])\right)\right. \\
&\left.\quad-\left.q(t-s, \sigma, \tau) a\left(X_{s}(\tau)\right)\right|^{2}\right] d \tau \\
& \leqq C_{1} \int_{0}^{t} d s \int_{0}^{1}\left\{q^{(N)}(t-s,[\sigma],[\tau])-q(t-s, \sigma, \tau)\right\}^{2} d \tau \\
&+C_{2} \int_{0}^{t} d s \int_{0}^{1} E\left[\left|X_{s}^{(N)}([\tau])-X_{s}(\tau)\right|^{2}\right] q^{2}(t-s, \sigma, \tau) d \tau .
\end{aligned}
$$

The discussion in Appendix II guarantees that the first term of the last line of (2.23) converges to 0 uniformly in $(t, \sigma) \in[0, T] \times[0,1]$ as $N \rightarrow \infty$. 
The expectation

$$
E\left[\left|X_{t, 3}^{(N)}([\sigma])-X_{t, 3}(\sigma)\right|^{2}\right]
$$

can be estimated in a similar way to get the estimate (2.23). Summing up, for each $\varepsilon>0$, there exists $N_{0} \in N$ such that

$$
\begin{aligned}
& \sup _{\sigma \in[0,1]} E\left[\left|X_{t}^{(N)}([\sigma])-X_{t}(\sigma)\right|^{2}\right] \\
& \leqq \varepsilon+C_{3} \int_{0}^{t}(t-s)^{-1 / 2} \sup _{\sigma \in[0,1]} E\left[\left|X_{s}^{(N)}([\sigma])-X_{s}(\sigma)\right|^{2}\right] d s, \\
& \qquad t \in[0, T], \quad \text { for every } N \geqq N_{0} .
\end{aligned}
$$

Using this estimate $n$ times over, we have

$$
\begin{aligned}
& \left\|X^{(N)}([\cdot])-X\right\|_{T}^{2} \leqq \varepsilon \sum_{i=0}^{n-1}\left(C_{3}^{2} \pi T\right)^{i / 2} \Gamma^{-1}(1+i / 2) \\
& +\left(C_{3}^{2} \pi T\right)^{n / 2} \Gamma^{-1}(1+n / 2)\left\|X^{(N)}([\cdot])-X\right\|_{T}^{2}
\end{aligned}
$$

where $\Gamma(x)$ is the gamma function. Noting that $\left\|X^{(N)}([\cdot])\right\|_{T}<\infty$ and $\|X\|_{T}$ $<\infty$, we have

$$
\left\|X^{(N)}([\cdot])-X\right\|_{T}^{2} \leqq \varepsilon C_{4}, \quad C_{4}=\sum_{i=0}^{\infty}\left(C_{3}^{2} \pi T\right)^{i / 2} \Gamma^{-1}(1+i / 2)<\infty .
$$

This proves

$$
\lim _{N \rightarrow \infty}\left\|X^{(N)}([\cdot])-X\right\|_{T}=0
$$

Noting the equality

$$
\begin{aligned}
& E\left[\left|X_{t}^{(N)}([\sigma])-X_{t}^{(N)}(\sigma)\right|^{2}\right] \\
& \quad=N^{2}([\sigma]-\sigma)^{2} E\left[\left|X_{t}^{(N)}\left([\sigma]-\frac{1}{N}\right)-X_{t}^{(N)}([\sigma])\right|^{2}\right],
\end{aligned}
$$

we have, by the representation (2.15) and Lemma 2.2,

$$
\lim _{N \rightarrow \infty}\left\|X^{(N)}([\cdot])-X^{(N)}\right\|_{T}=0 .
$$

By the equalities (2.24) and (2.25), we finally conclude

$$
\lim _{N \rightarrow \infty}\left\|X^{(N)}-X\right\|_{T}=0 \text {. }
$$

We are now ready to prove Theorem 2.1.

Proof of Theorem 2.1. Noting Lemma 2.2 and that $X_{t, 1}^{(N)}(\sigma)$ converges to $X_{t, 1}(\sigma)$ uniformly in $(t, \sigma)$ on each compact subset of $[0, \infty) \times[0,1]$ as 
$N \rightarrow \infty$, we see that $\left\{P^{(N)}\right\}_{N=1}^{\infty}$ is tight as a system of probability measures on $C\left([0, \infty) \times[0,1], R^{d}\right)$. Proposition 2.1 implies that every finite dimensional joint distribution of $P^{(N)}$ tends to that of $P$. Since $C([0, \infty) \times[0,1]$, $\left.R^{d}\right)=C([0, \infty), \mathscr{C})$ and these two spaces have equivalent topologies, we have the conclusion.

(Q.E.D.)

By Theorem 2.1, one may think of that the $\mathscr{C}$-valued stochastic differential equation (2.5) describes a mathematical model of an elastic string which moves in $R^{d}$ being interfered with by the diffusion process $x_{t}$. The following sections will be devoted to the discussions of several properties of the equation.

\section{$\S 3$. The limit of the solution as the modulus of elasticity $\kappa \rightarrow \infty$}

The solution $X_{t}(\sigma)$ of the equation (2.5) depends on the parameter $\kappa$, so that we denote the solution by $X_{t}(\sigma ; \kappa)$. The purpose of this section is to investigate the limit process of the $X_{t}(\sigma ; \kappa)$ as the modulus of elasticity $\kappa$ of the string tends to infinity. In fact, we shall prove the following results:

(i) In the case (I), the string shrinks to a single point whose motion is determined by the stochastic differential equation (2.1).

(ii) In the case (II), the string contracts to the point $A_{0}$.

(iii) In the case (III), the string converges to the segment $\overline{A_{0} A_{1}}$.

In the cases (II) and (III), we shall also study the deviation of the $X_{t}(\sigma ; \kappa)$ from the limit.

We denote by $P_{(\varepsilon)}$ the probability measure which is induced on $C((0, \infty), \mathscr{C})$ by $X_{t}(\sigma ; \kappa)$ and also by $X_{t, i}(\sigma ; \kappa)$ each of the three terms in the right hand side of the equation (2.7) instead of $X_{t, i}(\sigma)(i=1,2,3)$, respectively.

To prove the above results we prepare two lemmas which give estimates on $X_{t, i}(\sigma ; \kappa)(i=1,2,3)$. The $X_{t, 1}(\sigma ; \kappa)$ has the following representation:

$$
X_{t, 1}(\sigma ; \kappa)=\int_{0}^{1}\left\{X_{0}(\tau)-\tilde{X}(\tau)\right\} q(\kappa t, \sigma, \tau) d \tau+\tilde{X}(\sigma)
$$

where

$$
\tilde{X}(\sigma)=\left\{\begin{array}{cl}
0, & \text { in the case (I), } \\
A_{0}, & \text { in the case (II) }, \\
(1-\sigma) A_{0}+\sigma A_{1}, & \text { in the case (III). }
\end{array}\right.
$$


By this representation and the estimate (AII. 11) in Appendix II, we have the following lemma immediately.

Lemma 3.1. There exists a positive constant $C$ such that, for every $\kappa \geqq 1, t>0$ and $\sigma \in[0,1]$,

$$
\left|X_{t, 1}(\sigma ; \kappa)-\bar{x}_{0}\right| \leqq C e^{-\beta \kappa t} \quad \text { in the case }(\mathrm{I}) \text {, }
$$

and

$$
\left|X_{t, 1}(\sigma ; \kappa)-\tilde{X}(\sigma)\right| \leqq C e^{-\beta \kappa t} \quad \text { in the cases (II) and (III) }
$$

where

$$
\bar{x}_{0}=\int_{0}^{1} X_{0}(\sigma) d \sigma,
$$

and where $\beta$ is a positive constant which appears in (AII.8).

This lemma implies that

$$
\sup _{(t, \sigma, \kappa) \in[0, \infty) \times[0,1] \times[1, \infty)}\left|X_{t, 1}(\sigma ; \kappa)\right|<\infty .
$$

Lemma 3.2. For $i=2,3, p>0$ and $T<\infty$,

$$
\sup _{\kappa \geqq 1}\left\|X_{., i}(\cdot ; \kappa)\right\|_{T, p}<\infty \quad \text { in the case }(\mathrm{I}) \text {, }
$$

and

$$
\sup _{\kappa \geqq 1} \sqrt{\kappa}\left\|X_{., i}(\cdot ; \kappa)\right\|_{T, p}<\infty \quad \text { in the cases (II) and (III). }
$$

Proof. First, for $p \in N$, we prove by using Proposition 1.3,

$$
\begin{aligned}
& E\left[\left|X_{t, 2}(\sigma ; \kappa)\right|^{2 p}\right] \\
& \quad \leqq C_{1}\left\{\int_{0}^{t} d s E\left[\left(\int_{0}^{1}\left|a\left(X_{s}(\tau)\right)\right|^{2} q^{2}(\kappa(t-s), \sigma, \tau) d \tau\right)^{p}\right]^{1 / p}\right\}^{p} \\
& \quad \leqq C_{2}\left\{\int_{0}^{t} q(2 \kappa s, \sigma, \sigma) d s\right\}^{p} .
\end{aligned}
$$

In the case (I), we have by (AII.12),

$$
\begin{aligned}
& \int_{0}^{t} q(2 \kappa s, \sigma, \sigma) d s \leqq C_{3} \int_{0}^{t}\{1+1 / \sqrt{2 \kappa s}\} d s \\
& \quad=C_{3}\{t+\sqrt{2 t} / \sqrt{\kappa}\} \\
& \quad \leqq C_{3}\{T+\sqrt{2 T}\}, \quad t \leqq T, \quad \kappa \geqq 1 .
\end{aligned}
$$

While, in the cases (II) and (III), 


$$
\begin{gathered}
\int_{0}^{t} q(2 \kappa s, \sigma, \sigma) d s=\frac{1}{2 \kappa} \int_{0}^{2 \kappa t} q(u, \sigma, \sigma) d u \\
\leqq(2 \kappa)^{-1} G(\sigma, \sigma) \leqq C_{4} \kappa^{-1}
\end{gathered}
$$

where $G$ is the Green function of (1/2) $\Delta$ with the boundary conditions (2.9). Therefore, we have the desired estimates for $i=2$.

To derive estimates for $i=3$,

$$
\begin{aligned}
& E\left[\left|X_{t, 3}(\sigma ; \kappa)\right|^{2 p}\right] \\
& \quad \leqq E\left[\left(\int_{0}^{t} d s \int_{0}^{1}\left|b\left(X_{s}(\tau ; \kappa)\right)\right|^{2} d \tau\right)^{p}\right] \times\left\{\int_{0}^{t} d s \int_{0}^{1} q^{2}(\kappa(t-s), \sigma, \tau) d \tau\right\}^{p} \\
& \quad \leqq C_{5}\left\{\int_{0}^{t} q(2 \kappa s, \sigma, \sigma) d s\right\}^{p} t^{p-1} \int_{0}^{t}\left\{1+\sup _{\tau \in[0,1]} E\left[\left|X_{s}(\tau ; \kappa)\right|^{2 p}\right]\right\} d s .
\end{aligned}
$$

Noting (3.3) and estimates which are derived above for $i=2$, we have

$$
\begin{gathered}
\sup _{\sigma \in[0,1]} E\left[\left|X_{t}(\sigma ; \kappa)\right|^{2 p}\right] \leqq C_{6} \int_{0}^{t}\left\{1+\sup _{\tau \in[0,1]} E\left[\left|X_{s}(\tau ; \kappa)\right|^{2 p}\right]\right\} d s \\
\text { for } t \leqq T \text { and } \kappa \geqq 1 .
\end{gathered}
$$

By Gronwall's lemma, we have

$$
\sup _{\kappa \geq 1}\|X .(\cdot ; \kappa)\|_{T, 2 p}<\infty \text {. }
$$

Inserting this estimate into the right hand side of the inequality (3.6), by (3.4) and (3.5), we have the concluding estimates for $i=3$.

To show the tightness of the family $\{X .(\cdot ; \kappa), \kappa \geqq 1\}$, we need the following lemma.

Lemma 3.3. For $i=2,3$ and for each $T<\infty, p \in N$, there exists a positive constant $C$ such that

$$
\begin{aligned}
& E\left[\left|X_{t_{1}, i}\left(\sigma_{1} ; \kappa\right)-X_{t_{2}, i}\left(\sigma_{2} ; \kappa\right)\right|^{2 p}\right] \\
& \leqq C\left\{\left|t_{1}-t_{2}\right|^{p / 2}+\kappa^{-p}\left|\sigma_{1}-\sigma_{2}\right|^{p}\right\} \\
& \quad \text { for every } t_{1}, t_{2} \in[0, T], \sigma_{1}, \sigma_{2} \in[0,1], \quad \kappa \geqq 1 .
\end{aligned}
$$

Proof. For $0 \leqq t_{1} \leqq t_{2} \leqq T$ and $\sigma_{1}, \sigma_{2} \in[0,1]$, we have in a similar manner to the proof of Lemma 2.2,

$$
\begin{aligned}
& E\left[\left|X_{t_{1}, 2}\left(\sigma_{1} ; \kappa\right)-X_{t_{2}, 2}\left(\sigma_{2} ; \kappa\right)\right|^{2 p}\right] \\
& \qquad C_{1}\left\{\int_{0}^{t_{1}} q\left(2 \kappa s, \sigma_{1}, \sigma_{1}\right) d s+\int_{0}^{t_{2}} q\left(2 \kappa s, \sigma_{2}, \sigma_{2}\right) d s\right. \\
& \left.\quad-2 \int_{\left(t_{2}-t_{1}\right) / 2}^{\left(t_{1}+t_{2}\right) / 2} q\left(2 \kappa s, \sigma_{1}, \sigma_{2}\right) d s\right\}^{p}
\end{aligned}
$$




$$
\begin{aligned}
& \leqq C_{1}(2 \kappa)^{-p}\left\{\int_{0}^{2 \kappa t_{1}^{*}} q\left(u,{ }^{\prime} \sigma_{1},{ }_{-} \sigma_{1}\right) d u+\int_{0}^{2 \kappa t_{2}} q\left(u, \sigma_{2}, \sigma_{2}\right) d u\right. \\
& \left.-2 \int_{x\left(t_{2}-t_{1}\right)}^{x\left(t_{1}+t_{2}\right)} q\left(u, \sigma_{1}, \sigma_{2}\right) d u\right\}^{p} .
\end{aligned}
$$

The expression in braces is equal to

$$
\begin{aligned}
& G\left(2 \kappa t_{1}, \sigma_{1}, \sigma_{1}\right)+G\left(2 \kappa t_{1}, \sigma_{2}, \sigma_{2}\right)-2 G\left(2 \kappa t_{1}, \sigma_{1}, \sigma_{2}\right) \\
& +\int_{2 \kappa t_{1}}^{2 \kappa t_{2}} q\left(u, \sigma_{2}, \sigma_{2}\right) d u-2 \int_{2 \kappa t_{1}}^{\kappa\left(t_{1}+t_{2}\right)} q\left(u, \sigma_{1}, \sigma_{2}\right) d u \\
& \quad+2 \int_{0}^{\kappa\left(t_{2}-t_{1}\right)} q\left(u, \sigma_{1}, \sigma_{2}\right) d u,
\end{aligned}
$$

where $G(t ; \sigma, \tau)$ is a function which is defined in Appendix II. By the estimate (AII. 15) on $G(t ; \sigma, \tau)$, the absolute value of the sum of first three terms in (3.9) is bounded by $6\left|\sigma_{1}-\sigma_{2}\right|$ for every $\kappa>0$. The estimate (AII. 12) implies that there exists $C=C(T)>0$ such that the absolute value of the sum of remaining three terms in (3.9) is bounded by $C_{\kappa} \sqrt{t_{2}-t_{1}}$. Therefore, we have the estimate (3.8) for $i=2$.

In a similar way, we have the estimate (3.8) for $i=3$ by noting (3.7) in the proof of Lemma 3.2.

From now on, we shall discuss the problems in the case (I) and in the cases (II), (III), separately.

Case (I). Let $x_{t}$ be the solution of the stochastic differential equation (2.1) with a starting point $\bar{x}_{0}$. We define $X_{t}(\sigma ; \infty)$ by

$$
X_{t}(\sigma ; \infty)=x_{t} \quad \text { for }(t, \sigma) \in(0, \infty) \times[0,1] .
$$

Denote by $P_{(\infty)}$ the probability measure on $C((0, \infty), \mathscr{C})$ induced by $X_{t}(\sigma ; \infty)$. We show that $X_{t}(\cdot ; \kappa)$ tends to $X_{t}(\cdot ; \infty)$ as $\kappa \rightarrow \infty$ in the following sense.

Theorem 3.1. (i ) For $0<T_{1}<T_{2}<\infty$,

$$
\lim _{\kappa \rightarrow \infty} \sup _{(t, \sigma) \in\left[T_{1}, T_{2}\right] \times[0,1]} E\left[\left|X_{t}(\sigma ; \kappa)-X_{t}(\sigma ; \infty)\right|^{2}\right]=0,
$$

where $X_{t}(\sigma ; \infty)$ is defined as in (3.10) by the solution $x_{t}$ of the stochastic differential equation (2.1) with a Brownian motion $w_{t}=\left\langle B_{t}(\sigma), \chi_{[0,1]}(\sigma)\right\rangle$, when a cylindrical Brownian motion $B_{t}(\sigma)$ is given.

(ii) The probability measure $P_{(\kappa)}$ tends weakly to $P_{(\infty)}$ as $\kappa \rightarrow \infty$.

Proof. The process $x_{t}=X_{t}(\sigma ; \infty)$ satisfies the integral equation: 


$$
\begin{aligned}
x_{t} & =\int_{0}^{1} X_{0}(\tau) d \tau+\int_{0}^{t} \int_{0}^{1} a\left(x_{s}\right) d B_{s}(\tau) d \tau+\int_{0}^{t} \int_{0}^{1} b\left(x_{s}\right) d s d \tau \\
& \equiv \bar{x}_{0}+x_{t, 2}+x_{t, 3} .
\end{aligned}
$$

As for the process $X_{t}(\sigma ; \kappa)$,

$$
\begin{aligned}
& E\left[\left|X_{t, 2}(\sigma ; \kappa)-x_{t, 2}\right|^{2}\right] \\
& \leqq \\
& \leqq \int_{0}^{t} d s \int_{0}^{1} E\left[\left|a\left(X_{s}(\tau ; \kappa)\right) q(\kappa(t-s), \sigma, \tau)-a\left(x_{s}\right)\right|^{2}\right] d \tau \\
& \leqq 2 \int_{0}^{t} d s \int_{0}^{1}\left\{E\left[\left|a\left(X_{s}(\tau ; \kappa)\right)\right|^{2}\right](q(\kappa(t-s), \sigma, \tau)-1)^{2}\right. \\
& \left.\quad+E\left[\left|a\left(X_{s}(\tau ; \kappa)\right)-a\left(x_{s}\right)\right|^{2}\right]\right\} d \tau \\
& \leqq C_{1} \int_{0}^{t}\{q(2 \kappa s, \sigma, \sigma)-1\} d s+C_{1} \int_{0}^{t} \sup _{\tau \in[0,1]} E\left[\left|X_{s}(\tau ; \kappa)-x_{s}\right|^{2}\right] d s \\
& \leqq C_{2} \kappa^{-1}+C_{1} \int_{0}^{t} \sup _{\tau \in[0,1]} E\left[\left|X_{s}(\tau ; \kappa)-x_{s}\right|^{2}\right] d s .
\end{aligned}
$$

With the help of (3.7) with $p=1$, we have the estimate for $X_{t, 3}(\sigma ; \kappa)$ :

$$
\begin{aligned}
& E\left[\left|X_{t, 3}(\sigma ; \kappa)-x_{t, 3}\right|^{2}\right] \\
& \quad \leqq C_{3} \kappa^{-1}+C_{3} \int_{0}^{t} \sup _{\tau \in[0,1]} E\left[\left|X_{s}(\tau ; \kappa)-x_{s}\right|^{2}\right] d s .
\end{aligned}
$$

Again by (3.7), for each $\varepsilon>0$, there exists $\delta: 0<\delta<T_{1}$ such that

$$
\int_{0}^{\delta} \sup _{\tau \in[0,1]} E\left[\left|X_{s}(\tau ; \kappa)-x_{s}\right|^{2}\right] d s \leqq \varepsilon \quad \text { for every } \kappa \geqq 1 .
$$

By Lemma 3.1, there exists $\kappa_{0}>1 / \varepsilon$ such that

$$
\sup _{(t, o) \in\left[\hat{\sigma}, T_{2}\right] \times[0,1]}\left|X_{t, 1}(\sigma ; \kappa)-\bar{x}_{0}\right|^{2} \leqq \varepsilon, \quad \text { for } \kappa \geqq \kappa_{0} .
$$

The above estimates prove that

$$
\begin{gathered}
E\left[\left|X_{t}(\sigma ; \kappa)-x_{t}\right|^{2}\right] \leqq C \varepsilon+C \int_{\delta}^{t} \sup _{\tau \in[0,1]} E\left[\left|X_{s}(\tau ; \kappa)-x_{s}\right|^{2}\right] d s \\
\text { for } t \in\left[\delta, T_{2}\right], \quad \kappa \geqq \kappa_{0} .
\end{gathered}
$$

We therefore have

$$
\sup _{\sigma \in[0,1]} E\left[\left|X_{t}(\sigma ; \kappa)-x_{t}\right|^{2}\right] \leqq C \varepsilon e^{C(t-\hat{o})}, \quad t \in\left[\delta, T_{2}\right], \quad \kappa \geqq \kappa_{0},
$$

which implies the assertion (i).

In particular, we see that every finite dimensional joint distribution of $P_{(k)}$ converges to that of $P_{(\infty)}$. Since the family of probability measures 
$\left\{P_{(x)} ; \kappa \geqq 1\right\}$ is tight by Lemma 3.1 and Lemma 3.3 , we have the assertion (ii).

(Q.E.D.)

Cases (II) and (III). Set $X_{t}(\sigma ; \infty)=\tilde{X}(\sigma)$ for $(t, \sigma) \in(0, \infty) \times[0,1]$, where $\tilde{X}(\sigma)$ is defined as in (3.2), and denote by $P_{(\infty)}$ the probability measure on $C((0, \infty), \mathscr{C})$ induced by $X_{t}(\sigma ; \infty)$. In the cases (II) and (III), we can easily show that $X_{t}(\cdot ; \kappa)$ tends to $\tilde{X}(\cdot)$ as $\kappa \rightarrow \infty$, as is prescribed below.

TheOREM 3.2. (i) For $0<T_{1}<T_{2}<\infty$ and $p>0$, we have

$$
\lim _{\kappa \rightarrow \infty} \sup _{(t, \sigma) \in\left[T_{1}, T_{2}\right] \times[0,1]} E\left[\left|X_{t}(\sigma ; k)-\tilde{X}(\sigma)\right|^{2 p}\right]=0 .
$$

(ii) The probability measure $P_{(k)}$ converges weakly to $P_{(\infty)}$ as $\kappa \rightarrow \infty$.

Proof. The assertion (i) follows from Lemma 3.1 and Lemma 3.2, while the assertion (ii) follows by noting Lemma 3.3.

(Q.E.D.)

We shall also discuss the deviation of the $X_{t}(\sigma ; \kappa)$ from the limit $\tilde{X}(\cdot)$ :

$$
\lim _{k \rightarrow \infty} Y_{t}(\sigma ; \kappa),
$$

where

$$
Y_{t}(\sigma ; \kappa)=\sqrt{\kappa}\left(X_{t}(\sigma ; \kappa)-\tilde{X}(\sigma)\right)
$$

Setting

$$
\bar{Y}_{t, 2}(\sigma ; \kappa)=\sqrt{\kappa} \int_{0}^{t} \int_{0}^{1} a(\tilde{X}(\tau)) q(\kappa(t-s), \sigma, \tau) d B_{s}(\tau) d \tau,
$$

we have the following.

Lemma 3.4. For each $t>0$,

(i) $\lim _{s \rightarrow \infty} \sup _{\sigma \in[0,1]} E\left[\left|\sqrt{\kappa} X_{t, 2}(\sigma ; \kappa)-\bar{Y}_{t, 2}(\sigma ; \kappa)\right|^{2}\right]=0$,

(ii) $\lim _{\kappa \rightarrow \infty} \sup _{\sigma \in[0,1]} E\left[\left|\sqrt{\kappa} X_{t, 3}(\sigma ; \kappa)\right|^{2}\right]=0$.

Proof. Lemma 3.1, Lemma 3.2 and (AII. 12) prove

$$
\begin{aligned}
E[\mid & \left.\sqrt{\kappa} X_{t, 2}(\sigma ; \kappa)-\left.\bar{Y}_{t, 2}(\sigma ; \kappa)\right|^{2}\right] \\
& =\kappa \int_{0}^{t} d s E\left[\int_{0}^{1}\left|a\left(X_{s}(\tau ; \kappa)\right)-a(\tilde{X}(\tau))\right|^{2} q^{2}(\kappa(t-s), \sigma, \tau) d \tau\right] \\
& \leqq C_{1} \kappa \int_{0}^{t} \sup _{\tau \in[0,1]} E\left[\left|a\left(X_{s}(\tau ; \kappa)\right)-a(\tilde{X}(\tau))\right|^{2}\right] q(2 \kappa(t-s), \sigma, \sigma) d s \\
& \leqq C_{2} \kappa \int_{0}^{t}\left\{e^{-2 \beta \kappa s}+\kappa^{-1}\right\}(2 \kappa(t-s))^{-1 / 2} d s \\
& \rightarrow 0 \quad(\text { as } \kappa \rightarrow \infty) .
\end{aligned}
$$


This implies the assertion (i).

The estimate (AII. 11) on $q$ shows that

$$
\begin{aligned}
& E\left[\left|\sqrt{\kappa} X_{t, 3}(\sigma ; \kappa)\right|^{2}\right] \\
& \leqq C_{3} \kappa E\left[\left\{\int_{0}^{t} d s \int_{0}^{1}\left|X_{s}(\tau ; \kappa)-\tilde{X}(\tau)\right| q(\kappa(t-s), \sigma, \tau) d \tau\right\}^{2}\right] \\
& +2 \kappa\left\{\int_{0}^{t} d s \int_{0}^{1}|b(\tilde{X}(\tau))| q(\kappa(t-s), \sigma, \tau) d \tau\right\}^{2} \\
& \leqq C_{3} \kappa \int_{0}^{t} d s \int_{0}^{1} E\left[\left|X_{s}(\tau ; \kappa)-\tilde{X}(\tau)\right|^{2}\right] d \tau \times \int_{0}^{t} d s \int_{0}^{1} q^{2}(\kappa s, \sigma, \tau) d \tau \\
& +C_{4} \kappa\left\{\int_{0}^{t} d s \int_{0}^{1} q(\kappa s, \sigma, \tau) d \tau\right\}^{2} \\
& \leqq C_{3} G(\sigma, \sigma) \int_{0}^{t}\left(e^{-2 \beta \kappa s}+\kappa^{-1}\right) d s+C_{5} \kappa\left\{\int_{0}^{t} e^{-\beta \kappa s} d s\right\}^{2} \\
& \rightarrow 0 \quad(\text { as } \kappa \rightarrow \infty) \text {. }
\end{aligned}
$$

We have therefore proved the assertion (ii).

(Q.E.D.)

The next step is to show that $Y_{t}(\cdot ; \kappa)$ tends to a process $Y_{t}(\cdot)$ which is defined later. First, we consider a Gaussian $\mathscr{C}$-valued random variable $Y$ with mean 0 and covariance

$$
E\left[Y\left(\sigma_{1}\right) \otimes Y\left(\sigma_{2}\right)\right]=\left\{\begin{array}{lr}
\frac{1}{2}\left(a a^{*}\right)\left(A_{0}\right) G_{2}\left(\sigma_{1}, \sigma_{2}\right) & \text { in the case (II) } \\
\int_{0}^{\infty} d s \int_{0}^{1}\left(a a^{*}\right)(\tilde{X}(\tau)) q_{3}\left(s, \sigma_{1}, \tau\right) q_{3}\left(s, \sigma_{2}, \tau\right) d \tau & \text { in the case (III), }
\end{array}\right.
$$

where $Y_{1} \otimes Y_{2}=\left(\left(Y_{1} \otimes Y_{2}\right)_{i j}\right)=\left(Y_{1}^{i} Y_{2}^{j}\right)$ for vectors $Y_{1}=\left(Y_{1}^{i}\right)_{i=1}^{d}, \quad Y_{2}=\left(Y_{2}^{i}\right)_{\imath=1}^{d}$ and $a a^{*}=\left(\left(a a^{*}\right)_{i j}\right)=\left(\sum_{k=1}^{d} a_{i k} a_{j k}\right)$ for a matrix $a=\left(a_{i j}\right)$. The definitions of $G_{2}$ and $q_{3}$ can be found in Appendix II. Let $\left\{Y_{t} ; t>0\right\}$ be a family of independent $\mathscr{C}$-valued random variables such that each $Y_{t}$ has the same distribution as $Y$.

Remark 3.1. In the case (II), $Y(\cdot)$ has a realization: $Y(\sigma)=a\left(A_{0}\right) w_{\sigma}$ with a standard $d$-dimensional Brownian motion $w_{\sigma}$, with a time parameter $\sigma \in[0,1]$, starting from the origin. While, in the case (III), if $a(x)=a$ (constant matrix) for $\left[x \in{\overline{A_{0} A_{1}}}_{1}\right.$, then $Y(\sigma)=a w_{\sigma}$ with a $d$-dimensional Brownian bridge $w_{\sigma}$.

Lemma 3.5. For every $0<t_{1} \leqq t_{2} \leqq \cdots \leqq t_{n}$ and $\sigma_{i} \in[0,1] \quad(i=1$, $2, \cdots, n)$, the joint distribution of $\left\{\bar{Y}_{t_{i}, 2}\left(\sigma_{i} ; \kappa\right)\right\}_{i=1}^{n}$ on $\left(R^{d}\right)^{n}$ converges to that of $\left\{Y_{t_{i}}\left(\sigma_{i}\right)\right\}_{i=1}^{n}$ as $\kappa \rightarrow \infty$. 
Proof. Since $\left\{\bar{Y}_{t, 2}(\sigma ; \kappa) ;(t, \sigma) \in(0, \infty) \times[0,1]\right\}$ is a Gaussian system with mean 0 , it is enough to show the convergence of covariance functions. It is of the form

$$
\begin{aligned}
& E\left[\bar{Y}_{t_{1}, 2}\left(\sigma_{1} ; \kappa\right) \otimes \bar{Y}_{t_{2}, 2}\left(\sigma_{2} ; \kappa\right)\right] \\
& \quad=\kappa \int_{0}^{t_{1} \wedge t_{2}} d s \int_{0}^{1}\left(a a^{*}\right)(\tilde{X}(\tau)) q\left(\kappa\left(t_{1}-s\right), \sigma_{1}, \tau\right) q\left(\kappa\left(t_{2}-s\right), \sigma_{2}, \tau\right) d \tau .
\end{aligned}
$$

When $t_{1} \neq t_{2}$, by (AII. 8), the right hand side of (3.13) tends to 0 as $\kappa \rightarrow \infty$; and when $t_{1}=t_{2}$, it tends to $E\left[Y\left(\sigma_{1}\right) \otimes Y\left(\sigma_{2}\right)\right]$. Therefore, we have the conclusion.

(Q.E.D.)

THEOREM 3.3. For every $0<t_{1} \leqq t_{2} \leqq \cdots \leqq t_{n}$, the joint distribution of $\left\{Y_{t_{i}}(\cdot ; \kappa)\right\}_{i=1}^{n}$ on $\mathscr{C}^{n}$ tends to that of $\left\{Y_{t_{i}}(\cdot)\right\}_{i=1}^{n}$ as $\kappa \rightarrow \infty$.

Proof. By Lemma 3.1, for each $t>0$, we have

$$
\lim _{\kappa \rightarrow \infty} \sup _{\sigma \in[0,1]}\left|\sqrt{\kappa}\left(X_{t, 1}(\sigma ; \kappa)-\tilde{X}(\sigma)\right)\right|=0 .
$$

By Lemma 3.4, Lemma 3.5 and (3.14), we see that the joint distribution of $\left\{Y_{t_{i}}\left(\sigma_{i} ; \kappa\right)\right\}_{i=1}^{n}$ tends to that of $\left\{Y_{t_{i}}\left(\sigma_{i}\right)\right\}_{i=1}^{n}$ for every $0<t_{1} \leqq t_{2} \leqq \cdots \leqq t_{n}$ and $\sigma_{i} \in[0,1](i=1,2, \cdots, n)$. Taking $t_{1}=t_{2}=t$ in (3.8), we have

$$
\begin{gathered}
E\left[\left|\sqrt{\kappa} X_{t, i}\left(\sigma_{1} ; \kappa\right)-\sqrt{\kappa} X_{t, i}\left(\sigma_{2} ; \kappa\right)\right|^{2 p}\right] \leqq C\left|\sigma_{1}-\sigma_{2}\right|^{p} \\
\text { for } i=2,3 .
\end{gathered}
$$

Combining this with (3.14), we see that $\left\{Y_{t}(\cdot ; \kappa) ; \kappa \geqq 1\right\}$ is tight as a family of $\mathscr{C}$-valued random variables for each $t>0$. Therefore, we obtain the conclusion.

(Q.E.D.)

\section{§4. Brownian strings in a potential field}

In this section, we discuss the equation of a Brownian string in a potential field $U(x)\left(x \in R^{d}\right)$, that is, the equation (2.5) with $a(x)=I_{d}$ and $b(x)=-\nabla U(x) / 2$ :

$$
d X_{t}(\sigma)=d B_{t}(\sigma)-\frac{1}{2} \nabla U\left(X_{t}(\sigma)\right) d t+\frac{\kappa}{2} \Delta X_{t}(\sigma) d t
$$

We shall give a stationary measure of the equation (4.1) explicitly, and we shall also investigate the equation in the case where the potential function $U$ diverges on some region in $R^{d}$.

(a) The stationary measure of the equation (4.1).

As is stated in Introduction and will be proved in Appendix III, the 
equation (4.1) may be thought of as the Einstein-Smoluchowski equation on the space $\mathscr{C}$. In the finite dimensional case, A. N. Kolmogorov [11] characterized the equations of this type mathematically and gave their stationary measures. By analogy with his results, we may expect that the stationary measure of the equation (4.1) would be given by the following formal measure $\nu$ on $\mathscr{C}$ :

$$
d \nu(X)=e^{-H(X)} \mathscr{D}(X),
$$

where $H(X)$ is the Hamiltonian (0.3) of string and $\mathscr{D}(X)$ is the "Lebesgue measure" on $\mathscr{C}$ (Feynman measure).

To state more mathematically, define, for each of three cases (I)-(III), a $d$-dimensional process $w_{\sigma}, \sigma \in\left[0, \kappa^{-1}\right]$, as follows.

(i) In the case (I), $w_{\sigma}$ is a Brownian motion whose initial distribution is the Lebesgue measure on $R^{d}$.

(ii) In the case (II), $w_{\sigma}$ is a Brownian motion with a starting point $A_{0}$.

(iii) In the case (III), $w_{o}$ is a pinned Brownian motion which starts from $A_{0}$ and arrives at $A_{1}$.

We denote by $\mu_{(k)}$ a non-negative measure on $(\mathscr{C}, \mathscr{B}(\mathscr{C}))$ induced by the process $w_{\sigma / k}(\sigma \in[0,1])$, where $\mathscr{B}(\mathscr{C})$ is the topological $\sigma$-algebra on $\mathscr{C}$. As a mathematical realization of the formal measure in (4.2), we define a non-negative measure $\nu$ on $(\mathscr{C}, \mathscr{B}(\mathscr{C}))$ by

$$
d \nu(X)=\exp \left\{-\int_{0}^{1} U\left(X_{\sigma}\right) d \sigma\right\} d \mu_{(x)}(X) .
$$

Before we discuss the properties of $\nu$, we introduce some notations:

(i) $C_{0}\left(R^{d N}\right)=\left\{F \in C\left(R^{d N}\right) ; F\right.$ has a compact support $\}$,

(ii) $C_{b}(\mathscr{C})=\{f \in C(\mathscr{C}) ; f$ is bounded $\}$,

$$
C_{0}(\mathscr{C})=\left\{f \in C_{b}(\mathscr{C}) ; f \equiv 0 \text { on } \mathscr{C}_{M}^{c} \text { with some } 0<M<\infty\right\} \text {, }
$$

where

$$
\mathscr{C}_{\mathrm{MH}}=\{X \in \mathscr{C} ;|X(\sigma)| \leqq M \text { for every } \sigma \in[0,1]\},
$$

(iii) $\mathscr{D}_{N}=\left\{f \in C(\mathscr{C}) ; f(X)=F\left(\left\{X\left(\frac{k}{N}\right)\right\}_{k=1}^{N}\right)\right.$ with $\left.F \in C_{0}\left(R^{d N}\right)\right\}$,

$$
\mathscr{D}=\bigcup_{N=1}^{\infty} \mathscr{D}_{N} \text {. }
$$

The potential function $U$ will be assumed to satisfy the following conditions.

Assumption 1. The function $U$ belongs to $C^{1}\left(R^{a}\right), \nabla U$ is Lipschitz 
continuous and $U$ is bounded from below.

Under this assumption, the equation (4.1) has a unique solution $X_{t}(\sigma)$ and we can prove the following theorem.

Theorem 4.1. Under Assumption 1, for each of three cases (I)-(III), the measure $\nu$ is a reversible measure of the solution $X_{t}$ of (4.1), that is,

$$
\begin{gathered}
\int_{\mathscr{C}} E_{X_{0}}\left[f\left(X_{t}\right)\right] g\left(X_{0}\right) d \nu\left(X_{0}\right)=\int_{\mathscr{C}} E_{X_{0}}\left[g\left(X_{t}\right)\right] f\left(X_{0}\right) d \nu\left(X_{0}\right) \\
\text { holds for every } f, g \in C_{0}(\mathscr{C}),
\end{gathered}
$$

where $E_{X_{0}}[\cdot]$ means the expectation with respect to the probability measure induced on $C([0, \infty), \mathscr{C})$ by the solution $X_{t}$ of $(4.1)$ with initial state $X_{0} \in \mathscr{C}$.

Proof. We define a function $\bar{U}$ on $R^{d N}$ by

$$
\begin{gathered}
\bar{U}\left(X^{(N)}\right)=\sum_{k=1}^{N} U\left(X^{(N)}\left(\frac{k}{N}\right)\right)+\frac{\kappa N^{2}}{2} \sum_{k=1}^{N+1}\left|X^{(N)}\left(\frac{k-1}{N}\right)-X^{(N)}\left(\frac{k}{N}\right)\right|^{2}, \\
X^{(N)}=\left\{X^{(N)}\left(\frac{k}{N}\right)\right\}_{k=1}^{N} \in R^{d N},
\end{gathered}
$$

where $X^{(N)}(0)$ and $X^{(v)}((N+1) / N)$ satisfy the boundary conditions (2.4). Using the function $\bar{U}$, the equation (2.3) for $X_{t}^{(N)}=\left\{X_{t}^{(N)}(k / N)\right\}_{k=1}^{N}$ can be rewritten into the following form:

$$
d X_{t}^{(N)}\left(\frac{k}{N}\right)=\sqrt{N} d w_{t}(k)-\frac{1}{2} \nabla_{(k)} \bar{U}\left(X_{t}^{(N)}\right) d t .
$$

Define a non-negative measure $\nu_{N}$ on $R^{d N}$ by

$$
\begin{aligned}
& d \nu_{N}\left(X^{(N)}\right) \equiv \exp \left[-\frac{1}{N} \bar{U}\left(X^{(N)}\right)\right] d X^{(N)} \\
&=C_{N} \exp \left[-\frac{1}{N} \sum_{k=1}^{N} U\left(X^{(N)}\left(\frac{k}{N}\right)\right)\right] \\
& \quad \times \mu_{(\kappa)}\left(X\left(\frac{k}{N}\right) \in d X^{(N)}\left(\frac{k}{N}\right), 1 \leqq k \leqq N\right),
\end{aligned}
$$

where $d X^{(N)}$ is the Lebesgue measure on $R^{d N}$ and $C_{N}$ is an appropriate constant which depends on $N$. Then, it is known that $\nu_{N}$ is a reversible measure of (4.4), i.e.,

$$
\begin{aligned}
& \int_{R^{d N}} E_{X_{0}^{(N)}}\left[F\left(X_{t}^{(N)}\right)\right] G\left(X_{0}^{(N)}\right) d \nu_{N}\left(X_{0}^{(N)}\right) \\
&=\int_{R^{d N}} E_{X_{0}^{(N)}}\left[G\left(X_{t}^{(N)}\right)\right] F\left(X_{0}^{(N)}\right) d \nu_{N}\left(X_{0}^{(N)}\right) \\
& \text { holds for every } F, G \in C_{0}\left(R^{d N}\right) .
\end{aligned}
$$


We therefore have

$$
\begin{gathered}
\int_{\mathscr{C}} E_{X_{0}}^{(N)}\left[f\left(X_{t}\right)\right] g\left(X_{0}\right) \exp \left\{-\frac{1}{N} \sum_{k=1}^{N} U\left(X_{0}\left(\frac{k}{N}\right)\right)\right\} d \mu_{(k)}\left(X_{0}\right) \\
=\int_{\mathscr{C}} E_{X_{0}}^{(N)}\left[g\left(X_{t}\right)\right] f\left(X_{0}\right) \exp \left\{-\frac{1}{N} \sum_{k=1}^{N} U\left(X_{0}\left(\frac{k}{N}\right)\right)\right\} d \mu_{(\kappa)}\left(X_{0}\right) \\
\text { for every } f, g \in \mathscr{D}_{N}, \quad N=1,2, \cdots,
\end{gathered}
$$

where $E_{X_{0}}^{(N)}[\cdot]$ means the expectation with respect to the probability measure determined on $C([0, \infty), \mathscr{C})$ by $X_{t}^{(N)}$ as in $\S 2$. Noting Theorem 2.1 and that $U$ is bounded from below, we get the equality (4.3) for $f, g \in \mathscr{D}$ by taking the limit of both sides in the above equality. Approximating the functions in $C_{0}(\mathscr{C})$ by those in $\mathscr{D}$, we can easily show that the equality (4.3) holds for every $f, g \in C_{0}(\mathscr{C})$.

Remark 4.1. (i) We can show that the equality (4.3) holds for every $f, g \in C_{b}(\mathscr{C})$ in the cases (II) and (III).

(ii) Infinite-dimensional Einstein-Smoluchowski equations have been studied by several authors, e.g., H. Doss et G. Royer [7], R. Lang [13] and R. Marcus [15].

(b) The case with a divergent potential.

Here, we limit our discussions to the case (III). The potential function $U$ is permitted to diverge on some region in $R^{d}$. Namely, $U$ is a continuous function from $R^{d}$ into $(-\infty, \infty]$. Consider following regions in $R^{d}$ determined by $U$.

$$
\begin{aligned}
D & =\left\{x \in R^{d} ; U(x)<\infty\right\} \\
D_{N} & =\left\{x \in R^{a} ; U(x)<N\right\}, \quad N=1,2, \cdots .
\end{aligned}
$$

We set the following assumption on $U$.

Assumption 2. The function $U$ is in $C^{1}(D)$ and is bounded from below. $\quad \nabla U$ is Lipschitz continuous on $D_{N}$ for each $N=1,2, \ldots$. Two points $A_{0}$ and $A_{1}$ belong to the region $D$ and are able to be connected with a continuous curve in $D$.

For a potential $U$ which satisfies Assumption 2, we shall construct a process that may be thought of as a stationary solution of the equation (4.1). First, we take a sequence of functions $\left\{U^{(N)}\right\}_{N=1}^{\infty}$ on $R^{d}$ which satisfy the following three conditions. 


$$
\begin{cases}\text { (i) } U^{(N)} \text { satisfies Assumption } 1 \text { for each } N=1,2, \cdots \\ \text { (ii) } U^{(N)}(x)=U(x) \quad \text { for } x \in D_{N} \\ \text { (iii) } U^{(1)}(x) \leqq U^{(2)}(x) \leqq \ldots \quad \uparrow U(x)\end{cases}
$$

For each $N$, by Theorem 4.1, there exists a stationary solution of the equation (4.1) with $U$ replaced by $U^{(N)}$ and its stationary probability measure $\nu^{(N)}$ is given by

$$
d \nu^{(N)}(X)=Z_{N}^{-1} \exp \left\{-\int_{0}^{1} U^{(N)}(X(\sigma)) d \sigma\right\} d \mu_{(\kappa)}(X),
$$

where $Z_{N}$ is a normalizing constant. We denote by $P^{(N)}$ the probability measure on $C((-\infty, \infty), \mathscr{C})$ induced by this stationary solution.

To show the existence of a limit of the sequence $\left\{P^{(N)}\right\}$, we need another assumption on $U$.

Assumption 3. The sequence $\left\{U^{(N)}\right\}$ satisfies

$$
\sup _{N \in N} \int_{\mathscr{C}} \int_{0}^{1}\left|\nabla U^{(N)}(X(\sigma))\right|^{10} d \sigma d \nu^{(N)}(X)<\infty .
$$

We shall give an example of the potential $U$ which satisfies this assumption later (Example 4.1).

Proposition 4.1. Under Assumptions 2 and 3 , the family $\left\{P^{(N)}\right\}_{N=1}^{\infty}$ of probability measures on $C((-\infty, \infty), \mathscr{C})$ is tight. The stationary distribution $\nu$ of any limit $P \equiv P_{(\kappa)}$ of the sequence $\left\{P^{(N)}\right\}$ is given by

$$
d \nu(X)=Z^{-1} \exp \left\{-\int_{0}^{1} U(X(\sigma)) d \sigma\right\} d \mu_{(\kappa)}(X),
$$

where $Z$ is a normalizing constant.

Proof. Noting that $U^{(N)}(x)$ is increasing in $N$ for each $x \in R^{d}$, we can easily show that $\nu^{(N)}$ tends weakly to $\nu$ as $N \rightarrow \infty$.

Under the probability distribution $P^{(N)}$, the process $X_{t}$ satisfies the following integral equation for $t \geqq 0$.

$$
\begin{aligned}
X_{t}(\sigma)= & X_{t, 1}(\sigma)+\int_{0}^{t} \int_{0}^{1} p(t-s, \sigma, \tau) d B_{s}(\tau) d \tau \\
& -\frac{1}{2} \int_{0}^{t} \int_{0}^{1} p(t-s, \sigma, \tau) \nabla U^{(N)}\left(X_{s}(\tau)\right) d s d \tau \\
\equiv & X_{t, 1}(\sigma)+X_{t, 2}(\sigma)+X_{t, 3}(\sigma)
\end{aligned}
$$

where $X_{t, 1}(\sigma)$ is the solution of the heat equation (2.8) with a random 
initial state $X_{0}(\sigma)$ whose distribution on $\mathscr{C}$ is equal to $\nu^{(N)}$. To show the tightness of $\left\{P^{(N)}\right\}$, we need uniform estimates on the moments:

$$
\begin{aligned}
M^{(N)}= & E^{(N)}\left[\left|X_{t_{1}}\left(\sigma_{1}\right)-X_{t_{2}}\left(\sigma_{2}\right)\right|^{10}\right] \\
& \text { for } t_{1}, t_{2} \in R^{1}, \quad \sigma_{1}, \sigma_{2} \in[0,1],
\end{aligned}
$$

where $E^{(N)}[\cdot]$ means the expectation with respect to $P^{(N)}$. Since $P^{(N)}$ is stationary, we may estimate $M^{(N)}$ only for $1 \leqq t_{1} \leqq t_{2} \leqq T<\infty$. For this purpose, we set

$$
\begin{aligned}
M_{i}^{(N)}= & E^{(N)}\left[\left|X_{t_{1}, i}\left(\sigma_{1}\right)-X_{t_{2}, i}\left(\sigma_{2}\right)\right|^{10}\right] \\
& \text { for } i=1,2,3 .
\end{aligned}
$$

First, by the representation (3.1) of $X_{t, 1}$,

$$
\begin{aligned}
M_{1}^{(N)}= & E^{(N)}\left[\mid \int_{0}^{1}\left\{X_{0}(\tau)-\tilde{X}(\tau)\right\}\left\{p\left(t_{1}, \sigma_{1}, \tau\right)-p\left(t_{2}, \sigma_{2}, \tau\right)\right\} d \tau\right. \\
& \left.+\tilde{X}\left(\sigma_{1}\right)-\left.\tilde{X}\left(\sigma_{2}\right)\right|^{10}\right] \\
\leqq & C_{1} E^{(N)}\left[\int_{0}^{1}\left|X_{0}(\tau)-\tilde{X}(\tau)\right|^{10} d \tau\right] \\
& \times\left[\int_{0}^{1}\left\{p\left(t_{1}, \sigma_{1}, \tau\right)-p\left(t_{2}, \sigma_{2}, \tau\right)\right\}^{2} d \tau\right]^{5}+C_{1}\left|\sigma_{1}-\sigma_{2}\right|^{10} .
\end{aligned}
$$

In the above estimate,

$$
\begin{aligned}
& \sup _{N} E^{(N)}\left[\int_{0}^{1}\left|X_{0}(\tau)-\tilde{X}(\tau)\right|^{10} d \tau\right] \\
& \quad \leqq C_{2} \sup _{N} \int_{\mathscr{C}} \int_{0}^{1}|X(\tau)|^{10} d \tau d \nu^{(N)}(X)+C_{2} \int_{0}^{1}|\tilde{X}(\tau)|^{10} d \tau<\infty,
\end{aligned}
$$

and (AII. 10) proves

$$
\begin{aligned}
& \int_{0}^{1}\left\{p\left(t_{1}, \sigma_{1}, \tau\right)-p\left(t_{2}, \sigma_{2}, \tau\right)\right\}^{2} d \tau \\
& \quad=p\left(2 t_{1}, \sigma_{1}, \sigma_{1}\right)+p\left(2 t_{2}, \sigma_{2}, \sigma_{2}\right)-2 p\left(t_{1}+t_{2}, \sigma_{1}, \sigma_{2}\right) \\
& \quad \leqq C_{3}\left\{\left|t_{1}-t_{2}\right|+\left|\sigma_{1}-\sigma_{2}\right|\right\} .
\end{aligned}
$$

Therefore, we have

$$
M_{1}^{(N)} \leqq C_{4}\left\{\left|t_{1}-t_{2}\right|+\left|\sigma_{1}-\sigma_{2}\right|\right\}^{5} .
$$

Next, by Lemma 3.3,

$$
M_{2}^{(N)} \leqq C_{5}\left\{\left|t_{1}-t_{2}\right|^{5 / 2}+\left|\sigma_{1}-\sigma_{2}\right|^{5}\right\}
$$

Finally, by Assumption 3, we get 


$$
\begin{aligned}
M_{3}^{(N)}= & 2^{-10} E^{(N)}\left[\left\{\int_{0}^{t_{2}} d s \int_{0}^{1} \nabla U^{(N)}\left(X_{s}(\tau)\right)\right.\right. \\
& \left.\left.\times\left(p\left(t_{1}-s, \sigma_{1}, \tau\right) \chi_{\left\{s \leqq t_{1}\right\}}-p\left(t_{2}-s, \sigma_{2}, \tau\right)\right) d \tau\right\}^{10}\right] \\
\leqq & C_{6} \int_{0}^{t_{2}} d s E^{(N)}\left[\left\{\int_{0}^{1}\left|\nabla U^{(N)}\left(X_{s}(\tau)\right)\right|^{2} d \tau\right\}^{5}\right] \\
& \times\left\{\int_{0}^{1}\left(p\left(t_{1}-s, \sigma_{1}, \tau\right) \chi_{\left\{s \leqq t_{1}\right\}}-p\left(t_{2}-s, \sigma_{2}, \tau\right)\right)^{2} d \tau\right\}^{5} \\
\leqq & C_{7}\left\{\int_{0}^{t_{1}} p\left(2 s, \sigma_{1}, \sigma_{1}\right) d s+\int_{0}^{t_{2}} p\left(2 s, \sigma_{2}, \sigma_{2}\right) d s\right. \\
& \left.-2 \int_{\left(t_{2}-t_{1}\right) / 2}^{\left(t_{1}+t_{2}\right) / 2} p\left(2 s, \sigma_{1}, \sigma_{2}\right) d s\right\}^{5} \\
\leqq & C_{8}\left\{\left|t_{1}-t_{2}\right|^{5 / 2}+\left|\sigma_{1}-\sigma_{2}\right|^{5}\right\} .
\end{aligned}
$$

Combining the estimates (4.6)-(4.8), we have

$$
M^{(N)} \leqq C\left\{\left|t_{1}-t_{2}\right|^{5 / 2}+\left|\sigma_{1}-\sigma_{2}\right|^{5 / 2}\right\},
$$

with a positive constant $C$ which is independent of $N$. Therefore, noting that $\nu^{(N)} \rightarrow \nu$ (weakly) as $N \rightarrow \infty$, we see that the sequence $\left\{P^{(N)}\right\}$ is tight and that the stationary measure of any limit $P$ of $\left\{P^{(N)}\right\}$ is equal to $\nu$.

(Q.E.D.)

We may regard a limit $P \equiv P_{(x)}$ of the sequence $\left\{P^{(N)}\right\}$ as a distribution of a stationary solution of the equation (4.1) with a divergent potential $U$. We give a decomposition of $P$ associated with the 1-dimensional homotopy class of the closure $\bar{D}$ of the region $D$ in the following manner.

Consider a subset of $\mathscr{C}$ :

$$
\begin{aligned}
\mathscr{C}\left(\bar{D} ; A_{0}, A_{1}\right)=\{X \in \mathscr{C} ; X(\sigma) \in \bar{D} \text { for every } \sigma & \in[0,1] \text { and } \\
X(0) & \left.=A_{0}, X(1)=A_{1}\right\} .
\end{aligned}
$$

The set $\mathscr{C}\left(\bar{D} ; A_{0}, A_{1}\right)$ can be decomposed into connected components $\mathscr{C}_{i}$ $\left(i \in \pi_{1}\right)$ :

$$
\mathscr{C}\left(\bar{D} ; A_{0}, A_{1}\right)=\bigcup_{i \in \pi_{1}} \mathscr{C}_{i}, \quad \mathscr{C}_{i} \in \mathscr{B}(\mathscr{C}) .
$$

The set $\pi_{1}$ above is the fundamental group of the connected component of $\bar{D}$ which includes two points $A_{0}$ and $A_{1}$. Since the support of the probability measure $\nu$ is included in $\mathscr{C}\left(\bar{D} ; A_{0}, A_{1}\right)$, we have the following decomposition of $P$. 
TheOREM 4.2. The probability measure $P$ has a decomposition:

$$
P=\sum_{i \in \pi_{1} ; a_{i}>0} a_{i} P_{i}
$$

where $a_{i}=\nu\left(\mathscr{C}_{i}\right)$ and $P_{i}=P_{i,(x)}$ is a conditional probability measure $P\left(\cdot \mid C\left((-\infty, \infty), \mathscr{C}_{i}\right)\right)$.

Finally in this part, we give an example of a potential function $U$ which satisfies Assumption 3.

ExAmple 4.1. The following radial function $U$ on $R^{2}$ satisfies Assumption 3 for every $\kappa>0$.

$$
U(x)=U(r)= \begin{cases}\infty & \text { for } r \leqq r_{0} \\ \left(r-r_{0}\right)^{-\alpha} & \text { for } r>r_{0}\end{cases}
$$

with some $0<r_{0}<\infty$ and $\alpha>2$, where $r=|x|$.

Let $\mu_{0, A,(s)}^{0, \tau}$ be a probability distribution of a 2-dimensional pinned Brownian motion with speed $1 / \kappa$ which starts from 0 at time 0 and arrives at $A$ at time $\tau$. Noting that, with a standard Brownian motion $w(\sigma)$, the process $X(\sigma)$ defined by

$$
X(\sigma)=w\left(\frac{\sigma}{\kappa}\right)+\frac{\sigma}{\tau}\left(-w\left(\frac{\sigma}{\kappa}\right)+A\right), \quad \sigma \in[0, \tau],
$$

has the distribution $\mu_{0, A,(x)}^{0, \tau}$, we have the following lemma.

Lemma 4.1. For each $\kappa>0, p \in N$ and each bounded set $E$ in $R^{2}$, there exists $C=C(\kappa, p)>0$ such that

$$
\begin{aligned}
& \sup _{\tau \in(0,1]} \sup _{A \in E} \mu_{0, A,(\kappa)}^{0, \tau}[|\{\sigma \in[0, \tau] ; X(\sigma) \in D(0, a)\}|<b] \\
& \leqq C\left(b a^{-2}\right)^{p} \quad \text { for every } a, b ; 0<b \leqq a \leqq 1,
\end{aligned}
$$

where $D(0, a)=\left\{x \in R^{2} ;|x|<a\right\}$.

With this lemma, we show that the function $U$ in Example 4.1 satisfies Assumption 3. For the function $U$, we consider a sequence $\left\{U^{(n)}\right\}_{n=1}^{\infty}$ of functions which satisfy the conditions (i) and (iii) in (4.5) and the following (iv) and (v).

$$
U^{(n)}(x)= \begin{cases}U(x), & x \in \bigcup_{k=1}^{n} R_{k} \\ \text { constant, } & x \in \bigcup_{k=n+2}^{\infty} R_{k} \cup D^{c},\end{cases}
$$


(v)

$$
\left|\nabla U^{(n)}(x)\right| \leqq C_{1} k^{\alpha+1}, \quad x \in R_{k}, \quad 1 \leqq k \leqq n+1,
$$

where

$$
\begin{aligned}
& R_{1}=\left\{x \in R^{2} ;|x| \geqq r_{0}+1\right\}, \\
& R_{k}=\left\{x \in R^{2} ; r_{0}+\frac{1}{k} \leqq|x| \leqq r_{0}+\frac{1}{k-1}\right\}, \quad k=2,3, \cdots .
\end{aligned}
$$

For our purpose, it is now enough to show that

$$
\sup _{n \geq 1} \sup _{\tau \in[0,1]} I_{n}(\tau)<\infty,
$$

where

$$
I_{n}(\tau)=Z_{n}^{-1} \int_{\mathscr{\varepsilon}}\left|\nabla U^{(n)}\right|^{10}(X(\tau)) \exp \left[-\int_{0}^{1} U^{(n)}(X(\sigma)) d \sigma\right] d \mu_{(\kappa)}(X) .
$$

We first note that

$$
\begin{aligned}
& Z_{n} \equiv \int_{\mathscr{\ell}} \exp \left[-\int_{0}^{1} U^{(n)}(X(\sigma)) d \sigma\right] d \mu_{(\kappa)}(X) \\
& \geqq Z \equiv \int_{\mathscr{\wp}} \exp \left[-\int_{0}^{1} U(X(\sigma)) d \sigma\right] d \mu_{(x)}(X)>0 .
\end{aligned}
$$

For $\tau \in[1 / 2,1], I_{n}(\tau)$ can be estimated as follows.

$$
\begin{aligned}
& I_{n}(\tau) \leqq \sum_{k=1}^{n+1} \sup _{A \in R_{k}}\left|\nabla U^{(n)}\right|^{10}(A) Z_{n}^{-1} \int_{\varnothing} \exp \left[-\int_{0}^{\tau} U^{(n)}(X(\sigma)) d \sigma\right] d \mu_{\left.A_{0}, \theta_{,}, \tau\right)}(X) \\
& \times \int_{\mathscr{\varepsilon}} \exp \left[-\int_{\tau}^{1} U^{(n)}(X(\sigma)) d \sigma\right] d \mu_{A, A_{1},(\kappa)}^{\tau, 1}(X) \times \mu_{(\tau)}\left(X(\tau) \in R_{k}\right) \\
& \leqq Z^{-1} \sum_{k=1}^{n+1}\left(C_{1} k^{\alpha+1}\right)^{10} \sup _{A \in R_{k}} \int_{\mathscr{\varepsilon}} \exp \left[-\int_{0}^{\tau} U^{(n)}(X(\sigma)) d \sigma\right] d \mu_{A_{0}, A,(x)}^{0, \tau}(X) .
\end{aligned}
$$

To obtain an upper bound of the last integral, we consider the following two subsets of $\Omega=C\left([0, \tau], R^{d}\right)$ :

$$
\begin{aligned}
& \Omega_{1}=\left\{X \in \Omega ;|\{\sigma \in[0, \tau] ; X(\sigma) \in D(A, 1 / k)\}| \geqq 1 / k^{\beta}\right\}, \quad 2<\beta<\alpha, \\
& \Omega_{2}=\Omega-\Omega_{1} .
\end{aligned}
$$

Then,

$$
\begin{aligned}
\int_{\mathscr{8}} \exp & {\left[-\int_{0}^{\tau} U^{(n)}(X(\sigma)) d \sigma\right] d \mu_{A_{0}, A,(x)}^{0,} } \\
= & \int_{\Omega_{1}} \exp \left[-\int_{0}^{\tau} U^{(n)}(X(\sigma)) d \sigma\right] d \mu_{A_{0}, A,(x)}^{0, \tau} \\
& +\int_{\Omega_{2}} \exp \left[-\int_{0}^{\tau} U^{(n)}(X(\sigma)) d \sigma\right] d \mu_{A, \alpha,(x)}^{0, \tau} .
\end{aligned}
$$


The first integral in the right hand side of (4.11) is bounded by

$$
\exp \left[-(k-1)^{\alpha} / k^{\beta}\right] \leqq C_{2} \exp \left[-k^{\alpha-\beta}\right] \text {. }
$$

While, the second integral is bounded by

$$
\begin{aligned}
& \mu_{A, A_{0},(k)}^{0, \tau}\left(\Omega_{2}\right) \\
& \quad \leqq \sup _{A \in D\left(0,\left|A_{0}\right|+r_{0}+1\right)} \mu_{0, A,(k)}^{0, \tau}\left[|\{\sigma \in[0, \tau] ; X(\sigma) \in D(0,1 / k)\}|<1 / k^{\beta}\right] \\
& \quad \leqq C_{3}\left(k^{2} / k^{\beta}\right)^{p} .
\end{aligned}
$$

Hence, for $p$ large enough, we have

$$
I_{n}(\tau) \leqq C_{4} \sum_{k=1}^{\infty} k^{10(\alpha+1)}\left\{\exp \left(-k^{\alpha-\beta}\right)+k^{-p(\beta-2)}\right\}<\infty \quad \text { for } \tau \in[1 / 2,1] .
$$

For $\tau \in[0,1 / 2]$, exchanging the roles of $A_{0}$ and $A_{1}$, we have a similar estimate. We therefore have the estimate (4.9) which implies that the function $U$ in Example 4.1 satisfies Assumption 3.

(c) The limit of $P_{i,(x)}$ as $\kappa \rightarrow \infty$.

Here, we assume that $d=2$ and $D$ is a non-convex connected open polygon in $R^{2}$. We denote by $\mu_{i,(k)}$ and $\nu_{i,(k)}\left(i \in \pi_{1}\right)$ the conditional probability measures $\mu_{(\kappa)}\left(\cdot \mid \mathscr{C}_{i}\right)$ and $\nu_{(x)}\left(\cdot \mid \mathscr{C}_{i}\right)$, respectively. Let $\tilde{D}$ be the universal covering space of $\bar{D}$ and let $\tilde{p}(t, x, y)(t>0, x, y \in \tilde{D})$ be a transition probability density of the Brownian motion on $\tilde{D}$ with absorbing boundary $\partial \tilde{D}$. We note that there exists only one shortest continuous path $\tilde{\gamma}(x, y)$ in $\tilde{D}$ which joins two points $x, y \in \tilde{D}$. To investigate the limits of $\nu_{i,(x)}$ and $P_{i,(x)}$ as $\kappa \rightarrow \infty$, we use the following lemma and its corollary which were shown by S. A. Molchanov [17].

Lemma 4.2. Assume that $\tilde{\gamma}(x, y)$ is transversal to $\partial \tilde{D}$. Then,

$$
\tilde{p}(t, x, y)=O\left(t^{k-1} \exp \left\{-\frac{1}{2 t} \hat{\rho}(x, y)^{2}\right\}\right) \quad \text { as } t \rightarrow 0,
$$

where $k$ is a positive constant which depends on $\tilde{\gamma}(x, y)$ and $\hat{\rho}(\cdot, \cdot)$ is the metric on $\tilde{D}$.

$$
\begin{aligned}
& \text { For } \kappa \geqq 1 \text { and } 0<\beta<1 / 2, \text { we set } \\
& \Omega_{1}(\kappa)=\left\{X \in \mathscr{C} ; \operatorname{dis}(X(\sigma), \partial D)>\kappa^{-1 / 2} \quad \text { for every } \sigma \in[0,1]\right\} \\
& \Omega_{2}(\kappa, \beta)=\left\{X \in \mathscr{C} ; \operatorname{dis}\left(X(\sigma), \gamma_{i}(\sigma)\right)>\kappa^{-1 / 2+\beta} \quad \text { for some } \sigma \in[0,1]\right\},
\end{aligned}
$$

where $\gamma_{i}(\cdot)$ is the shortest continuous path in $\mathscr{C}_{i}$ which has a representation in terms of length and satisfies $\gamma_{i}(0)=A_{0}$ and $\gamma_{i}(1)=A_{1}$. 
CoRollary (1) $\varliminf_{k \rightarrow \infty} \mu_{i,(k)}\left(\Omega_{1}(\kappa)\right)>0$, for each $i \in \pi_{1}$.

(2) For each $0<\beta<1 / 2, \mu_{i,(\kappa)}\left(\Omega_{2}(\kappa, \beta)\right)=o\left(e^{-\kappa^{-2 \beta / 2}}\right)$ as $\kappa \rightarrow \infty$.

By the estimates in this corollary, we see that $\mu_{i,(x)}$ tends to $\delta_{r_{i}}$ (the $\delta$-measure concentrated on $\gamma_{i}$ ) as $\kappa \rightarrow \infty$. To show that $\nu_{i,(\kappa)} \rightarrow \delta_{r_{i}}$ holds as $\kappa \rightarrow \infty$, we need assume that $U$ and the region $D$ satisfy the following two conditions.

(vi) $U(x) \leqq C(\operatorname{dis}(x, \partial D))^{-\alpha}, x \in D$ with some $C>0$ and $0<\alpha<1+\sqrt{3}$. (vii) There exists a finite set $\left\{\sigma_{1}, \cdots, \sigma_{n}\right\}$ in the interval $(0,1)$ such that

$$
\begin{array}{ll}
\gamma_{i}\left(\sigma_{j}\right) \in \partial D & \text { for } j=1,2, \cdots, n, \\
\gamma_{i}(\sigma) \oplus \partial D & \text { for } \sigma \in[0,1]-\left\{\sigma_{1}, \cdots, \sigma_{n}\right\} .
\end{array}
$$

Then, we obtain the following.

Proposition 4.2. Under Assumptions 2, 3 and conditions (vi), (vii), we have

(1) $\nu_{i,(k)} \longrightarrow \delta_{r_{i}}$ as $\kappa \longrightarrow \infty$,

(2) $\quad P_{i,(\kappa)} \longrightarrow \delta_{X !, i}^{(\infty)}$ as $\kappa \longrightarrow \infty$,

where

$$
X_{t, i}^{(\infty)}(\sigma)=\gamma_{i}(\sigma) \quad \text { for every } t \in R^{1} .
$$

Proof. First, we estimate the normalizing constant $Z_{i,(\kappa)}$ :

$$
Z_{i,(\kappa)} \equiv \int_{\mathscr{Q}_{i}} \exp \left[-\int_{0}^{1} U(X(\sigma)) d \sigma\right] d \mu_{i,(\kappa)}(X) \text {. }
$$

We set $\Omega_{3}(\kappa, \beta) \equiv \Omega_{1}(\kappa) \cap \Omega_{2}^{c}(\kappa, \beta)$ with $\beta=1 / 2(\alpha+1)(0<\beta<1 / 2)$. Then, there exists a positive constant $c$ such that the inequality

$$
\operatorname{dis}(X(\sigma), \partial D)>\kappa^{-1 / 2+\beta}
$$

holds for $X \in \Omega_{3}(\kappa, \beta)$ and for $\sigma \in[0,1]:\left|\sigma-\sigma_{j}\right|>c \kappa^{-1 / 2+\beta}$ for any $j=1$, $2, \cdots, n$. Therefore, for such $X$ and $\sigma$ we have

$$
U(X(\sigma)) \leqq C \kappa^{(1 / 2-\beta) \alpha} .
$$

While, for $\sigma \in[0,1]$ such that $\left|\sigma-\sigma_{j}\right|<c \kappa^{-1 / 2+\beta}$ with some $j=1,2, \cdots, n$, the inequality

$$
U(X(\sigma)) \leqq C \kappa^{\alpha / 2}
$$

holds. Therefore, we get

$$
\int_{0}^{1} U(X(\sigma)) d \sigma \leqq C_{1}\left\{\kappa^{(1 / 2-\beta) \alpha}+\kappa^{\alpha / 2+\beta-1 / 2}\right\}=2 C_{1} \kappa^{\alpha^{2 / 2(\alpha+1)}} \quad \text { for } X \in \Omega_{3}(\kappa, \beta)
$$


Noting that

$$
\varliminf_{\kappa \rightarrow \infty} \mu_{i,(\kappa)}\left(\Omega_{3}(\kappa, \beta)\right)>0,
$$

we have

$$
Z_{i,(\kappa)} \geqq C_{2} \exp \left\{-2 C_{1} \kappa^{\alpha^{2 / 2(\alpha+1)}}\right\} \quad \text { for sufficiently large } \kappa .
$$

For each $\varepsilon: 0<\varepsilon<1 / 2$, since $U$ is bounded from below, the above two estimates prove

$$
\begin{aligned}
\nu_{i,(\kappa)}\left(\Omega_{2}(\kappa, \varepsilon)\right) & =Z_{i,(\kappa)}^{-1} \int_{\Omega_{2}(\kappa, \varepsilon)} \exp \left[-\int_{0}^{1} U(X(\sigma)) d \sigma\right] d \mu_{i,(\kappa)}(X) \\
& \leqq C_{3} \exp \left\{2 C_{1} \kappa^{\left.\alpha^{2 / 2(\alpha+1)}\right\}} \exp \left\{-\kappa^{-2 \varepsilon} / 2\right\} \quad(\kappa \rightarrow \infty) .\right.
\end{aligned}
$$

By taking $\varepsilon$ such that $1 / 2>\varepsilon>\alpha^{2} / 4(\alpha+1)$, we obtain

$$
\nu_{i,(\kappa)}\left(\Omega_{2}(\kappa, \varepsilon)\right) \longrightarrow 0 \quad \text { as } \kappa \longrightarrow \infty \text {. }
$$

This implies the assertion (1). The assertion (2) follows from the assertion

(1) immediately.

(Q.E.D.)

\section{§5. Two dimensional Brownian strings}

In this section, we assume that $d=2, \kappa=1, a(x)=I_{2}$ and $b(x)=0$. The equation (2.5) becomes as follows.

$$
d X_{t}(\sigma)=d B_{t}(\sigma)+\frac{1}{2} \Delta X_{t}(\sigma) d t
$$

We discuss the equation only in the case (I), namely the case where the equation has the Neumann boundary conditions at $\sigma=0$ and 1 . Let $P_{X_{0}}$ be a probability measure which is induced on $C([0, \infty), \mathscr{C})$ by the solution $X_{t}$ of the equation (5.1) with an initial state $X_{0} \in \mathscr{C}$. We now show the following recurrent properties of the solution $X_{t}$.

TheOREM 5.1. The process $X_{t}$ is recurrent as a $\mathscr{C}$-valued process, i.e., for each $X_{0} \in \mathscr{C}$ and non-empty open subset $O$ of $C$,

$$
P_{X_{0}}\left(X_{t_{n}} \in O \text { for some } t_{n} \uparrow \infty, n=1,2, \cdots\right)=1 \text {. }
$$

TheOREm 5.2. The string $X_{t}(\sigma)$ sweeps away all points in $R^{2}$, i.e., for every $x \in R^{2}$ and $X_{0} \in \mathscr{C}$,

$$
P_{X_{0}}\left(X_{t_{n}}\left(\sigma_{n}\right)=x \text { for some } \sigma_{n} \in[0,1] \text { and } t_{n} \uparrow \infty, n=1,2, \cdots\right)=1 \text {. }
$$

The integral form (2.7) of the equation (5.1) has already given the solution $X_{t}(\sigma)=\left\{X_{t}^{i}(\sigma)\right\}_{i=1}^{2}$ : 


$$
\begin{aligned}
X_{t}(\sigma) & =\int_{0}^{1} X_{0}(\tau) q(t, \sigma, \tau) d \tau+\int_{0}^{t} \int_{0}^{1} q(t-s, \sigma, \tau) d B_{s}(\tau) d \tau \\
& =X_{t, 1}(\sigma)+X_{t, 2}(\sigma) .
\end{aligned}
$$

The second term $X_{t, 2}(\sigma)$ can be decomposed as follows.

$$
\begin{aligned}
X_{t, 2}(\sigma) & =\left\langle B_{t}(\sigma), \chi_{[0,1]}(\sigma)\right\rangle+\int_{0}^{t} \int_{0}^{1}\{q(t-s, \sigma, \tau)-1\} d B_{s}(\tau) d \tau \\
& =Y_{t}+Z_{t}(\sigma) .
\end{aligned}
$$

In this decomposition, two processes $Y_{t}$ and $Z_{t}(\cdot)$ are mutually independent and $Y_{t}$ is a 2-dimensional Brownian motion.

Lemma 5.1. For each $t>0$, let $\nu_{t}$ be a probability measure on $\mathscr{C}=$ $C\left([0,1], R^{2}\right)$ induced by $Z_{t}(\sigma)(\sigma \in[0,1])$, and let $\nu$ be a probability measure on $\mathscr{C}$ induced by $\left\{w(\sigma)-\int_{0}^{1} w(\tau) d \tau ; \sigma \in[0,1]\right\}$, where $w(\sigma)$ is a 2-dimensional Brownian motion with a time parameter $\sigma \in[0,1]$. Then, $\nu_{t}$ tends weakly to $\nu$ as $t \rightarrow \infty$.

Proof. For $\sigma_{1}, \sigma_{2} \in[0,1], t>0$, noting (AII. 13), we have

$$
\begin{aligned}
& E\left[\left|Z_{t}\left(\sigma_{1}\right)-Z_{t}\left(\sigma_{2}\right)\right|^{4}\right] \\
& \quad=6\left\{\int_{0}^{t} d s \int_{0}^{1}\left(q\left(t-s, \sigma_{1}, \tau\right)-q\left(t-s, \sigma_{2}, \tau\right)\right)^{2} d \tau\right\}^{2} \\
& \quad \leqq \frac{3}{2}\left\{G\left(\sigma_{1}, \sigma_{1}\right)+G\left(\sigma_{2}, \sigma_{2}\right)-2 G\left(\sigma_{1}, \sigma_{2}\right)\right\}^{2} \\
& \quad=6\left|\sigma_{1}-\sigma_{2}\right|^{2} .
\end{aligned}
$$

For $t>0$,

$$
E\left[\left|Z_{t}(0)\right|^{2}\right]=2 \int_{0}^{t} d s \int_{0}^{1}\{q(t-s, 0, \tau)-1\}^{2} d \tau \leqq \frac{2}{3}
$$

Two estimates (5.4) and (5.5) imply that the family $\left\{\nu_{t}, t>0\right\}$ of probability measures is tight. To get the conclusion, noting that $\nu_{t}$ and $\nu$ are both Gaussian, it is enough to show that the covariance function of $\nu_{t}$ converges to that of $\nu$ as $t \rightarrow \infty$. For $\sigma_{1}, \sigma_{2} \in[0,1], i, j \in\{1,2\}$,

$$
\begin{aligned}
& E\left[Z_{t}^{i}\left(\sigma_{1}\right) Z_{t}^{j}\left(\sigma_{2}\right)\right] \\
& \quad=\delta_{i j} \int_{0}^{t} d s \int_{0}^{1}\left\{q\left(t-s, \sigma_{1}, \tau\right)-1\right\}\left\{q\left(t-s, \sigma_{2}, \tau\right)-1\right\} d \tau \\
& \quad=\frac{1}{2} \delta_{i j} \int_{0}^{2 t}\left\{q\left(s, \sigma_{1}, \sigma_{2}\right)-1\right\} d s
\end{aligned}
$$




$$
\begin{aligned}
& \rightarrow \frac{1}{2} \delta_{i j} G\left(\sigma_{1}, \sigma_{2}\right) \\
& =E\left[\left\{w^{i}\left(\sigma_{1}\right)-\int_{0}^{1} w^{i}(\tau) d \tau\right\}\left\{w^{j}\left(\sigma_{2}\right)-\int_{0}^{1} w^{j}(\tau) d \tau\right\}\right], \\
& \text { as } t \longrightarrow \infty .
\end{aligned}
$$

Therefore, we have the conclusion.

Now, we prove the theorems.

Proof of Theorem 5.1. We may assume that $O$ is taken to be

$$
D(\phi, a)=\left\{\psi \in \mathscr{C} ; \sup _{\sigma \in[0,1]} \psi(\sigma)-\phi(\sigma) \mid<a\right\}
$$

with some $\phi \in C$ and $a>0$. Fix a point $A$ in $R^{2}$ and we set

$$
V=D(A, a / 3)=\left\{A^{\prime} \in R^{2} ;\left|A-A^{\prime}\right|<a / 3\right\},
$$

and

$$
\begin{aligned}
t & =\inf \left\{t \geqq 0 ; X_{t} \in O\right\} \\
\bar{t} & =\inf \left\{t \geqq 0 ; \bar{X}_{t} \equiv \int_{0}^{1} X_{t}(\tau) d \tau \in V\right\} \\
\bar{t}_{N} & =\inf \left\{t \geqq N ; \bar{X}_{t} \in V\right\},
\end{aligned}
$$

where we tacitly understand that $\inf \{$ empty set $\}=\infty$. Since $\bar{X}_{t}$ is a 2dimensional Brownian motion, we have $N \leqq \bar{t}_{N}<\infty$ (a.s.). For every $X_{0}$ $\in \mathscr{C}$ such that $\bar{X}_{0}=\int_{0}^{1} X_{0}(\tau) d \tau \in V$,

$$
\begin{aligned}
& P_{X_{0}}(t<\infty) \\
& \geqq P_{X_{0}}\left(\text { there exists } t \geqq T_{0}\left(X_{0}\right) \text { such that } X_{t, 2} \in D(\phi-A, a / 3)\right) \\
& \geqq P_{X_{0}} \text { (there exists } t \geqq T_{0}\left(X_{0}\right) \text { such that } Y_{t} \in D(\bar{\phi}-A, a / 6) \\
& \text { and } \left.Z_{t} \in D(\phi-\bar{\phi}, a / 6)\right) \\
& \geqq \varlimsup_{N \rightarrow \infty} P_{X_{0}}\left(Z_{m_{N}} \in D(\phi-\bar{\phi}, a / 6)\right) \\
& =P\left(\left\{w(\cdot)-\int_{0}^{1} w(\tau) d \tau\right\} \in D(\phi-\bar{\phi}, a / 6)\right)=c>0,
\end{aligned}
$$

where $\phi-A \in \mathscr{C}$ is defined by $(\phi-A)(\sigma)=\phi(\sigma)-A, \bar{\phi} \equiv \int_{0}^{1} \phi(\sigma) d \sigma$,

$$
T_{0}\left(X_{0}\right)=\inf \left\{t>0 ; \sup _{\sigma \in[0,1]}\left|\int_{0}^{1} X_{0}(\tau) q(s, \sigma, \tau) d \tau-\bar{X}_{0}\right|<a / 3 \text { for every } s>t\right\}
$$


and

$$
m_{N}=\inf \left\{t \geqq N ; Y_{t} \in D(\bar{\phi}-A, a / 6)\right\} .
$$

The estimate (5.6) shows that

$$
p=\sup _{X_{0} \in \mathscr{\mathscr { C }} ; \mathbb{X}_{0} \in V} P_{X_{0}}(t=\infty) \leqq 1-c<1 .
$$

Using the strong Markov property of $X_{t}$ (Appendix I), we have

$$
\begin{aligned}
p & =\sup _{X_{0} \in \mathscr{G} ; \bar{X}_{0} \in V} \lim _{N \rightarrow \infty} P_{X_{0}}\left(t>\bar{t}_{N}, t=\infty\right) \\
& =\sup _{X_{0} \in \mathscr{C} ; \bar{X}_{0} \in V} \lim _{N \rightarrow \infty} E_{X_{0}}\left[P_{X_{t_{N}}}(t=\infty) ; t>\overline{\boldsymbol{t}}_{N}\right] \\
& \leqq p \sup _{X_{0} \in \mathscr{Y} ; X_{0} \in V} \lim _{N \rightarrow \infty} P_{X_{0}}\left(\boldsymbol{t}>\overline{\boldsymbol{t}}_{N}\right)=p^{2} .
\end{aligned}
$$

This implies, by (5.7), that $p=0$. Therefore, we have

$$
P_{X_{0}}(t<\infty)=1 \quad \text { for every } X_{0} \in \mathscr{C} \text {. }
$$

Noting the strong Markov property of $X_{t}$ again, we have the conclusion.

(Q.E.D.)

Corollary 5.1. For each non-empty open subset $O$ of $R^{2}$,

$$
\begin{gathered}
P_{X_{0}}\left(\left\{X_{t_{n}}(\sigma) ; \sigma \in[0,1]\right\} \subset O \text { for some } t_{n} \uparrow \infty, n=1,2, \cdots\right)=1 \\
\text { for every } X_{0} \in \mathscr{C} .
\end{gathered}
$$

Proof. Since the set $\{X \in \mathscr{C} ;\{X(\sigma) ; \sigma \in[0,1]\} \subset O\}$ is open in $\mathscr{C}$, we have the conclusion by Theorem 5.1 .

(Q.E.D.)

For $A=\left\{A^{i}\right\}_{i=1}^{2} \in R^{2}, a>0, T>0$ and $c, a>c>0$, we consider the following subset of $\mathscr{C}$ :

$$
\Phi(A, a, T, c)=\left\{\begin{array}{ll}
X=\left\{X^{i}\right\}_{i=1}^{2} \in \mathscr{C} & \begin{array}{l}
X \in D(A, a) \text { and for every } t \in[0, T], \\
\int_{0}^{1} X^{2}(\tau) q(t, 0, \tau) d \tau>A^{2}+c \\
\int_{0}^{1} X^{2}(\tau) q(t, 1, \tau) d \tau<A^{2}-c
\end{array}
\end{array}\right\} .
$$

Since, by taking $c$ sufficiently small, the set $\Phi(A, a, T, c)$ contains some non-empty open subset of $\mathscr{C}$, we have the following corollary.

Corollary 5.2. For every $A, a, T$ and $X_{0} \in \mathscr{C}$, there exists $c>0$ such that

$$
P_{X_{0}}\left(X_{t_{n}} \in \Phi(A, a, T, c) \text { for some } t_{n} \uparrow \infty, n=1,2, \cdots\right)=1
$$


Remark 5.1. When $d \geqq 3$, the process $\bar{X}_{t}$ on $R^{d}$ is not recurrent, so that we can show that the solution $X_{t}$ of (5.1) is also not recurrent as a $\mathscr{C}$-valued process.

Proof of Theorem 5.2. Since the equation (5.1) is invariant under the parallel displacement, we may assume that $x=0 \in R^{2}$. For $A=(-1,0)$ $\in R^{2}$ and $a>1$, we denote $\Phi(A, 1, T, c)$ simply by $\Phi(T, c)$, where $T$ is a constant determined later and $c$ is a positive number which is determined by Corollary 5.2. Set

$$
t=\inf \left\{t \geqq 0 ; X_{t}(\sigma)=0 \text { for some } \sigma \in[0,1]\right\},
$$

and

$$
p=\sup _{X_{0} \in \Phi(T, c)} P_{X_{0}}(t=\infty)
$$

For every $X_{0} \in \Phi(T, c)$, we have

$$
\begin{aligned}
& P_{X_{0}}(t<\infty) \\
& \geqq P_{X_{0}}\left\{\inf _{\sigma \in[0,1]} X_{T}^{1}(\sigma) \geqq 0, \inf _{t \in[0, T]} X_{t}^{2}(0) \geqq 0, \sup _{t \in[0, T]} X_{t}^{2}(1) \leqq 0\right\} \\
& \geqq P_{X_{0}}\left\{\inf _{\sigma \in[0,1]} X_{T, 2}^{1}(\sigma) \geqq 2\right\} \\
& \times P_{X_{0}}\left\{\inf _{t \in[0, T]} X_{t, 2}^{2}(0) \geqq-c, \sup _{t \in[0, T]} X_{t, 2}^{2}(1) \leqq c\right\} .
\end{aligned}
$$

The factor $p_{1}(T) \equiv P_{X_{0}}\left\{\inf _{\sigma \in[0,1]} X_{T, 2}^{1}(\sigma) \geqq 2\right\}$ can be estimated by the following way.

$$
\begin{aligned}
p_{1}(T) & \geqq P\left(Y_{T}^{1} \geqq 3, \inf _{\sigma \in[0,1]} Z_{T}^{1}(\sigma) \geqq-1\right) \\
& =P\left(Y_{T}^{1} \geqq 3\right) P\left(\inf _{\sigma \in[0,1]} Z_{T}^{1}(\sigma) \geqq-1\right) .
\end{aligned}
$$

Since $\lim _{T \rightarrow \infty} P\left(Y_{T}^{1} \geqq 3\right)=1 / 2$ and since $\lim _{T \rightarrow \infty} P\left(\inf _{\sigma \in[0,1]} Z_{T}^{1}(\sigma) \geqq-1\right)>0$ by Lemma 5.1, we can take $T<\infty$ such that $p_{1}(T)>0$. To estimate the second term:

$$
p_{2}(T) \equiv P_{X_{0}}\left\{\inf _{t \in[0, T]} X_{t, 2}^{2}(0) \geqq-c, \sup _{t \in[0, T]} X_{t, 2}^{2}(1) \leqq c\right\},
$$

we set

$$
y_{i}(t)=\frac{1}{2} \int_{0}^{t} \int_{0}^{1}\left\{q(t-s, 1, \tau)-(-1)^{i} q(t-s, 0, \tau)\right\} d B_{s}^{2}(\tau) d \tau, \quad i=1,2 .
$$

Then, two processes $y_{1}(t)$ and $y_{2}(t)$ are mutually independent. Noting that $X_{t, 2}^{2}(0)=y_{1}(t)-y_{2}(t)$ and $X_{t, 2}^{2}(1)=y_{1}(t)+y_{2}(t)$, 


$$
\begin{aligned}
p_{2}(T) & \geqq P\left\{\sup _{t \in[0, T]}\left|y_{1}(t)\right| \leqq c / 2, \sup _{t \in[0, T]}\left|y_{2}(t)\right| \leqq c / 2\right\} \\
& =\prod_{i=1}^{2} P\left\{\sup _{t \in[0, T]}\left|y_{i}(t)\right| \leqq c / 2\right\}>0 .
\end{aligned}
$$

Hence, we have $p<1$. Noting that, by Corollary 5.2, $N \leqq \bar{t}_{N}<\infty$ (a.s.) holds for $\bar{t}_{N}=\inf \left\{t \geqq N ; X_{t} \in \Phi(T, c)\right\}$, a similar argument to the proof of Theorem 5.1 leads us to the desired conclusion.

(Q.E.D.)

Remark 5.2. A generalization of the equation (5.1) is the Langevin equation on a Hilbert space $H$ :

$$
d X_{t}=d B_{t}-A X_{t} d t
$$

where $B_{t}$ is a cylindrical Brownian motion on $H$ and $A$ is an operator on $H$ which satisfies the condition (A.1) in $\S 1$. Concerning the equation (5.9), we can show the following two facts.

(i) If the third eigenvalue $\lambda_{3}$ of the operator $A$ is positive, then the solution $X_{t}$ of (5.9) is recurrent as an $H$-valued stochastic process.

(ii) If $\lambda_{3}=0$, then $X_{t}$ is transient (i.e., $\lim _{t \rightarrow \infty}\left\|X_{t}\right\|=\infty$ a.s.).

The third eigenvalue of (1/2) $\Delta$ is to be positive if it is regarded as an operator on $L^{2}\left([0,1], R^{2}\right)$, therefore we see that the solution $X_{t}$ of the equation (5.1) viewed as an $L^{2}\left([0,1], R^{2}\right)$-valued process is recurrent. This fact however does not imply Theorem 5.1 .

\section{§6. Limit theorems for general stochastic evolution equations}

As a generalization of the problem discussed in $\S 3$, we investigate the limit process of the solution $X_{t}^{(\kappa)}$ of the following stochastic differential equation (6.1) on a separable real Hilbert space $H$ as $\kappa \rightarrow \infty$.

$$
d X_{t}^{(\kappa)}=a\left(X_{t}^{(\kappa)}\right) d B_{t}+b\left(X_{t}^{(\kappa)}\right)-\kappa A X_{t}^{(\kappa)} d t .
$$

In the above equation, the operator $A$ is assumed to satisfy the assumption (A.1) in $\S 1$ and $B_{t}$ is the cylindrical Brownian motion. The coefficients $a$ and $b$ are assumed to be independent of $t$ and to satisfy the assumptions (A.2) and (A.3), respectively. In addition, we assume the following.

(A.4) There exists a positive constant $K$ such that

$$
\|a(X)\|_{\mathscr{L}(H)} \leqq K, \quad\|b(X)\| \leqq K \quad \text { for every } X \in H,
$$

where $\|a\|_{\mathscr{L}(H)}$ is the operator norm of $a$.

Let $\lambda$ and $n$ be the principal eigenvalue of $A$ and its multiplicity, respectively. We denote by $P_{r}^{[i, j]}(1 \leqq i \leqq j \leqq \infty)$ the orthogonal projection 
from $H$ into its subspace spanned by $\left\{\phi_{k}\right\}_{k=i}^{j}$, where $\phi_{k}$ is given in $\S 1$. We denote $P_{r}^{[1, n]}$ and $P_{r}^{[n+1, \infty]}$ simply by $P_{r}$ and $P_{r}^{\perp}$, respectively. We discuss the problems in the following four cases, separately.

(Case 1) $\lambda=0$

(Case 2) $\lambda>0, \quad a(0) \neq 0$

(Case 3) $\lambda>0, \quad a(0)=0, \quad b(0) \neq 0$

(Case 4) $\lambda>0, a(0)=0, \quad b(0)=0$.

Case 1. For given $X_{0} \in H$, consider the following stochastic differential equation:

$$
d Y_{t}=P_{r} a\left(Y_{t}\right) d B_{t}+P_{r} b\left(Y_{t}\right) d t, \quad Y_{0}=P_{r} X_{0} .
$$

Lemma 6.1. The equation (6.2) has a unique solution $Y_{t}$ which belongs to $C\left([0, \infty), P_{r} H\right)$ (a.s.).

Proof. The coefficients $P_{r} a$ and $P_{r} b$ of (6.2) are Lipschitz continuous in the following senses, respectively.

$$
\begin{aligned}
& \left\|P_{r} a\left(Y_{1}\right)-P_{r} a\left(Y_{2}\right)\right\|_{2}^{2} \\
& \quad=\sum_{k=1}^{\infty}\left\|\left\{P_{r} a\left(Y_{1}\right)-P_{r} a\left(Y_{2}\right)\right\} \phi_{k}\right\|^{2} \\
& \quad=\sum_{k=1}^{\infty}\left\|\left\{P_{r} a\left(Y_{1}\right)-P_{r} a\left(Y_{2}\right)\right\}^{*} \phi_{k}\right\|^{2} \\
& \quad=\sum_{k=1}^{n}\left\|\left\{a^{*}\left(Y_{1}\right)-a^{*}\left(Y_{2}\right)\right\} \phi_{k}\right\|^{2} \\
& \quad \leqq n K^{2}\left\|Y_{1}-Y_{2}\right\|^{2}
\end{aligned}
$$

and

$$
\left\|P_{r} b\left(Y_{1}\right)-P_{r} b\left(Y_{2}\right)\right\| \leqq K\left\|Y_{1}-Y_{2}\right\| \text {. }
$$

Hence, by M. Yor [19], there exists a unique solution $Y_{t} \in C([0, \infty), H)$ (a.s.) of the equation (6.2). Since $Y_{t}=P_{r} Y_{t}$ holds, the solution $Y_{t}$ lives in the space $P_{r} H$. We therefore have the conclusion.

(Q.E.D.)

Noting that the operator $-\kappa A$ generates a semi-group $\{U(\kappa t) ; t \geqq 0\}$, $X_{t}^{(s)}$ is a solution of the following integral equation:

$$
\begin{aligned}
X_{t}^{(k)} & =U(\kappa t) X_{0}+\int_{0}^{t} U(\kappa(t-s)) a\left(X_{s}^{(s)}\right) d B_{s}+\int_{0}^{t} U(\kappa(t-s)) b\left(X_{s}^{(k)}\right) d s \\
& \equiv X_{t, 1}^{(k)}+X_{t, 2}^{(k)}+X_{t, 3}^{(k)} .
\end{aligned}
$$

LEMmA 6.2. For each $p \geqq 1$, there exist $C, \tilde{C}>0$ such that 


$$
E\left[\left\|X_{t}^{(\kappa)}-Y_{t}\right\|^{2 p}\right] \leqq C\left(e^{-\tilde{C}_{\kappa} t}+\kappa^{-p} e^{C t}\right), \quad \text { for } t \in[0, \infty), \quad \kappa \geqq 1 .
$$

Proof. Set

$$
I^{(\kappa)}(t)=E\left[\left\|X_{t}^{(\kappa)}-Y_{t}\right\|^{2 p}\right]^{1 / p},
$$

and

$$
I_{i}^{(\kappa)}(t)=E\left[\left\|X_{t, i}^{(\kappa)}-Y_{t, i}\right\|^{2 p}\right] \quad(i=1,2,3),
$$

where

$$
Y_{t, 1}=P_{r} X_{0}, \quad Y_{t, 2}=\int_{0}^{t} P_{r} a\left(Y_{s}\right) d B_{s} \quad \text { and } \quad Y_{t, 3}=\int_{0}^{t} P_{r} b\left(Y_{s}\right) d s .
$$

As for the $I_{1}^{(\kappa)}(t)$,

$$
I_{1}^{(\kappa)}(t) \leqq \exp \left\{-\lambda_{n+1} 2 p \kappa t\right\}\left\|X_{0}\right\|^{2 p}
$$

While, by using Proposition 1.3,

$$
\begin{aligned}
I_{2}^{(\kappa)}(t) \leqq & 2^{2 p-1}\left\{E\left[\left\|\int_{0}^{t}\left\{U(\kappa(t-s))-P_{r}\right\} a\left(X_{s}^{(\kappa)}\right) d B_{s}\right\|^{2 p}\right]\right. \\
& \left.+E\left[\left\|\int_{0}^{t} P_{r}\left\{a\left(X_{s}^{(s)}\right)-a\left(Y_{s}\right)\right\} d B_{s}\right\|^{2 p}\right]\right\} \\
\leqq & \left.\quad+\left\{\int_{1}\left[\left\{\int_{0}^{t} E\left[\left\|\left(U(\kappa(t-s))-P_{r}\right) a\left(X_{s}^{(s)}\right)\right\|_{2}^{2 p}\right]^{1 / p} d s\right\}^{p}\left\{a\left(X_{s}^{(s)}\right)-a\left(Y_{s}\right)\right\} \|_{2}^{2 p}\right]^{1 / p} d s\right\}^{p}\right] \\
\leqq & C_{1}\left\{\left(\int_{0}^{t} \sum_{k=n+1}^{\infty} K^{2} \exp \left\{-2 \lambda_{k} k(t-s)\right\} d s\right)^{p}+\left(\int_{0}^{t} n K^{2} I^{(\kappa)}(s) d s\right)^{p}\right\} \\
\leqq & C_{2}\left\{\kappa^{-p}+\left(\int_{0}^{t} I^{(\kappa)}(s) d s\right)^{p}\right\}
\end{aligned}
$$

In a similar way, we can estimate the $I_{3}^{(\kappa)}(t)$.

Summing up the estimates on $I_{i}^{(\kappa)}(t)(i=1,2,3)$, we have

$$
I^{(\kappa)}(t) \leqq C_{3}\left\{\exp \left(-2 \lambda_{n+1} \kappa t\right)+\kappa^{-1}+\int_{0}^{t} I^{(x)}(s) d s\right\}
$$

We therefore get the desired estimate with the help of Gronwall's lemma (see, e.g., H. Kuo [12]).

The following estimate on a 1-dimensional stochastic integral was shown by G. Newell (see D. A. Dawson [5]). 
Lemma 6.3. Let $w_{t}$ be a 1-dimensional Brownian motion. Consider a stochastic integral

$$
x(t)=\int_{0}^{t} e^{-\lambda(t-s)} h(s) d w_{s},
$$

for $\lambda>0$ and for a real valued measurable function $h(t)=h(t, \omega)$ which is adapted to the Brownian motion $w_{t}$ and which satisfies

$$
|h(t)| \leqq M(\text { a.s. }) \quad \text { for every } t \in[0, T]
$$

Then, there exists $K_{1}>0$ which is independent of $\lambda, T$ and $M$, such that

$$
\begin{gathered}
P\left(\sup _{t \in[0, T]}|x(t)|>x\right) \leqq K_{1} \exp \left\{-\lambda x^{2} / 3 M^{2}\right\}(\lambda T+1) \\
\quad \text { for every } x>M K_{1} / \sqrt{\lambda}, \quad T \in[0, \infty) .
\end{gathered}
$$

Using this lemma, we can show the following.

Lemma 6.4. (i) There exist $C, \tilde{C}>0$ such that

$$
\begin{gathered}
P\left(\sup _{t \in[0, T]}\left\|P_{r}^{\perp} X_{t, 2}^{(\kappa)}\right\|>M\right) \leqq C e^{-\tilde{C} M^{2} \kappa}, \quad \text { for } M>0 \text { and } \kappa>C M^{-2} . \\
\sup _{t \in[0, T]}\left\|P_{r}^{\perp} X_{t, 3}^{(\kappa)}\right\| \leqq K /\left(\lambda_{n+1} \kappa\right) \quad \text { (a.s.). }
\end{gathered}
$$

Proof. For simplicity, we denote $\left\langle X_{t, i}^{(k)}, \phi_{k}\right\rangle(i=1,2,3, k \in N)$ by $X_{t, i, k}^{(k)}$. Setting $C_{1}=\left(\sum_{k=n+1}^{\infty} k^{-(1+\varepsilon)}\right)^{-1}$ with some $\varepsilon, 0<\varepsilon<\delta$ ( $\delta$ is the positive number given in (A.1)), we have

$$
\begin{aligned}
& P\left(\sup _{t \in[0, T]}\left\|P_{r}^{\perp} X_{t, 2}^{(\kappa)}\right\|>M\right) \\
& \quad \leqq \sum_{k=n+1}^{\infty} P\left(\sup _{t \in[0, T]}\left|X_{t, 2, k}^{(k)}\right|>\left(C_{1} k^{-(1+\varepsilon)}\right)^{1 / 2} M\right) \\
& \quad \leqq \sum_{k=n+1}^{\infty} K_{1}\left(\lambda_{k} k T+1\right) \exp \left\{-\lambda_{k} k C_{1} M^{2} /\left(3 K^{2} k^{1+s}\right)\right\} \\
& \quad \leqq C e^{-\tilde{C} M^{2 k}}, \quad \text { for some } C, \tilde{C}>0,
\end{aligned}
$$

whenever $\sqrt{C_{1} k^{-(1+\varepsilon)}} M>K K_{1} / \sqrt{\lambda_{k} \kappa}$ holds for every $k \geqq n+1$. Therefore, the estimate (i) holds for $\kappa$ such that

$$
\kappa>\left(K K_{1} / M\right)^{2} C_{1}^{-1} \sup _{k \geqq n+1}\left\{k^{1+\varepsilon} / \lambda_{k}\right\}
$$

The estimate (ii) can be shown by 


$$
\begin{aligned}
\left\|P_{r}^{\perp} X_{t, 3}^{(\kappa)}\right\| & =\left\|P_{r}^{\perp} \int_{0}^{t} U(\kappa(t-s)) b\left(X_{s}^{(\kappa)}\right) d s\right\| \\
& \leqq \int_{0}^{t} \exp \left\{-\lambda_{n+1} \kappa s\right\} K d s \leqq K /\left(\lambda_{n+1} \kappa\right) .
\end{aligned}
$$

We are now ready to prove the following theorem.

TheOREm 6.1. The process $X_{s}^{(\alpha)}$ converges to $Y_{t}$ as $k \rightarrow \infty$ in the following sense:

$$
\begin{array}{r}
P\left(\lim _{x(\in N) \rightarrow \infty}\left\|X_{t}^{(\kappa)}-Y_{t}\right\|=0 \text { uniformly in } t\right. \text { on each compact } \\
\text { subset of }(0, \infty))=1 .
\end{array}
$$

Proof. Noting that

$$
X_{t, 2, k}^{(\kappa)}=\int_{0}^{t}\left\langle a^{*}\left(X_{s}^{(\kappa)}\right) \phi_{k}, d B_{s}\right\rangle \quad \text { and } \quad Y_{t, 2, k} \equiv \int_{0}^{t}\left\langle a^{*}\left(Y_{s}\right) \phi_{k}, d B_{s}\right\rangle
$$

are $\left\{\mathscr{F}_{t} ; t \geqq 0\right\}$-martingales for each $k, 1 \leqq k \leqq n$, by Proposition 1.3 and Lemma 6.2, we have

$$
\begin{aligned}
& E\left[\sup _{t \in[0, T]}\left|X_{t, 2, k}^{(\kappa)}-Y_{t, 2, k}\right|^{4}\right] \\
& \quad \leqq\left(\frac{4}{3}\right)^{4} E\left[\left|X_{T, 2, k}^{(\kappa)}-Y_{T, 2, k}\right|^{4}\right] \\
& \quad \leqq C_{1}\left\{\int_{0}^{T} E\left[\left\|X_{s}^{(\kappa)}-Y_{s}\right\|^{4}\right]^{1 / 2} d s\right\}^{2} \\
& \quad \leqq C_{1}\left\{\int_{0}^{T} \sqrt{C}\left(e^{-\tilde{C}_{\kappa s}}+\kappa^{-2} e^{C s}\right)^{1 / 2} d s\right\}^{2} \leqq C_{2} \kappa^{-2}
\end{aligned}
$$

Hence,

$$
\sum_{\kappa=1}^{\infty} P\left(\sup _{t \in[0, T]}\left|X_{t, 2, k}^{(\kappa)}-Y_{t, 2, k}\right|^{4}>\kappa^{-1 / 2}\right) \leqq \sum_{k=1}^{\infty} \sqrt{\kappa} C_{2} \kappa^{-2}<\infty,
$$

and this shows, with the help of Borel-Cantelli's lemma, that

$$
\begin{gathered}
P\left(\sup _{t \in[0, T]}\left|X_{t, 2, k}^{(\kappa)}-Y_{t, 2, k}\right|^{2}<\kappa^{-1 / 4} \text { as } k(\in N) \longrightarrow \infty\right)=1, \\
\text { for each } k ; 1 \leqq k \leqq n .
\end{gathered}
$$

Therefore, we have

$$
P\left(\sup _{t \in[0, T]}\left\|P_{r} X_{t, 2}^{(\kappa)}-Y_{t, 2}\right\| \longrightarrow 0 \text { as } \kappa(\in N) \longrightarrow \infty\right)=1 .
$$

In a similar way, we can show that 


$$
P\left(\sup _{t \in[0, T]}\left\|P_{r} X_{t, 3}^{(\kappa)}-Y_{t, 3}\right\| \longrightarrow 0 \text { as } \kappa(\in N) \longrightarrow \infty\right)=1 .
$$

While, by Lemma 6.4-(i), for sufficiently large $\kappa_{0}$,

$$
\sum_{\kappa=\kappa_{0}}^{\infty} P\left(\sup _{t \in[0, T]}\left\|P_{r}^{\perp} X_{t, 2}^{(\kappa)}\right\|>\kappa^{-1 / 4}\right) \leqq \sum_{\kappa=\kappa_{0}}^{\infty} C e^{-\tilde{c} \sqrt{\kappa}}<\infty,
$$

and this implies that

$$
P\left(\sup _{t \in[0, T]}\left\|P_{r}^{\perp} X_{t, 2}^{(\kappa)}\right\| \longrightarrow 0 \text { as } \kappa(\in N) \longrightarrow \infty\right)=1 .
$$

We also have, by Lemma 6.4-(ii),

$$
\sup _{t \in[0, T]}\left\|P_{r}^{\perp} X_{t, 3}^{(\kappa)}\right\| \longrightarrow 0 \quad \text { as } \kappa \longrightarrow \infty \quad \text { (a.s.). }
$$

Since $X_{t, 1}^{(\kappa)}$ tends to $P_{r} X_{0}$ as $\kappa \rightarrow \infty$ uniformly in $t$ on each compact subset of $(0, \infty)$, combining $(6.7)-(6.10)$, we have the conclusion.

(Q.E.D.)

Case 2. Let $\left\{Y_{t}, t>0\right\}$ be a family of independent $H$-valued random variables whose characteristic functionals are given by

$$
E\left[e^{i\left\langle Y_{t}, \phi\right\rangle}\right]=e^{-|\|\phi\||^{2 / 2}}, \quad \phi \in H, \quad t>0,
$$

where

$$
\||| \phi||^{2}=\sum_{j, k=1}^{\infty}\left\langle a^{*}(0) \phi_{j}, a^{*}(0) \phi_{k}\right\rangle\left\langle\phi, \phi_{j}\right\rangle\left\langle\phi, \phi_{k}\right\rangle /\left(\lambda_{j}+\lambda_{k}\right) .
$$

We note that $\|\cdot \cdot\|$ is a Hilbert-Schmidt semi-norm on $H$.

The following theorem holds.

Theorem 6.2. The process $\left\{\sqrt{\kappa} X_{t}^{(\kappa)} ; t>0\right\}$ tends to $\left\{Y_{t} ; t>0\right\}$ as $\kappa \rightarrow \infty$ in the following sense:

$$
\begin{aligned}
& \sum_{i=1}^{n}\left\langle\sqrt{\kappa} X_{t_{i}}^{(\kappa)}, \psi_{i}\right\rangle \longrightarrow \sum_{i=1}^{n}\left\langle Y_{t_{i}}, \psi_{i}\right\rangle \quad \text { in law as } \kappa \longrightarrow \infty, \\
& \text { for every } n \in N, \quad \psi_{i} \in H(1 \leqq i \leqq n) \text { and } \\
& 0<t_{1}<t_{2}<\ldots<t_{n}<\infty \text {. }
\end{aligned}
$$

To prove the theorem, we prepare an estimate on the $X_{t}^{(\kappa)}$.

LEMMA 6.5. $E\left[\left\|X_{t}^{(\kappa)}\right\|^{2}\right] \leqq C\left(e^{-2 \lambda \kappa t}+\kappa^{-1}\right), \kappa \geqq 1$.

Proof. By (6.5), we have

$$
\begin{aligned}
E\left[\left\|X_{t}^{(\kappa)}\right\|^{2}\right] \leqq 3\left\{\left\|U(\kappa t) X_{0}\right\|^{2}+E\left[\int_{0}^{t}\right.\right. & \left.\left\|U(\kappa(t-s)) a\left(X_{s}^{(\kappa)}\right)\right\|_{2}^{2} d s\right] \\
& \left.+E\left[\left(\int_{0}^{t}\left\|U(\kappa(t-s)) b\left(X_{s}^{(\kappa)}\right)\right\| d s\right)^{2}\right]\right\}
\end{aligned}
$$




$$
\begin{aligned}
& \leqq 3\left\{e^{-2 \lambda x t}\left\|X_{0}\right\|^{2}+K^{2} \int_{0}^{t} \sum_{k=1}^{\infty} e^{-2 \lambda k r s} d s+K^{2}\left(\int_{0}^{t} e^{-\kappa \lambda s} d s\right)^{2}\right\} \\
& \leqq C\left(e^{-2 \lambda x t}+\kappa^{-1}\right) .
\end{aligned}
$$

Proof of Theorem 6.2. First, we have

$$
\begin{gathered}
\left\|\sqrt{\kappa}\left(X_{t, 1}^{(\kappa)}+X_{t, 3}^{(\kappa)}\right)\right\| \leqq \sqrt{\kappa} e^{-\kappa \lambda t}\left\|X_{0}\right\|+\sqrt{\kappa} K \int_{0}^{t} e^{-\kappa \lambda s} d s \longrightarrow 0 \\
\text { as } \kappa \longrightarrow \infty, \quad \text { for each } t>0 .
\end{gathered}
$$

Secondly, by Lemma 6.5,

$$
\begin{aligned}
& E\left[\left\|\sqrt{\kappa} X_{t, 2}^{(\kappa)}-\sqrt{\kappa} \int_{0}^{t} U(\kappa(t-s)) a(0) d B_{s}\right\|^{2}\right] \\
& =\kappa E\left[\left\|\int_{0}^{t} U(\kappa(t-s))\left\{a\left(X_{s}^{(\kappa)}\right)-a(0)\right\} d B_{s}\right\|^{2}\right] \\
& \leqq \kappa K^{2} \int_{0}^{t} \sum_{k=1}^{\infty} e^{-2 \kappa \lambda_{k} s} E\left[\left\|X_{t-s}^{(\kappa)}\right\|^{2}\right] d s \\
& \leqq \kappa K^{2} C \sum_{k=1}^{\infty} \int_{0}^{t} e^{-2 \kappa \lambda_{k} s}\left\{e^{-2 \lambda \kappa(t-s)}+\kappa^{-1}\right\} d s \longrightarrow 0, \\
& \quad \text { as } \kappa \longrightarrow \infty, \quad \text { for each } t>0 .
\end{aligned}
$$

The above two estimates imply that the limit process of $\left\{\sqrt{\kappa} X_{t}^{(k)}, t>0\right\}$ is the same as that of $\left\{Y_{t}^{(\kappa)}, t>0\right\}$ with $Y_{t}^{(\kappa)} \equiv \sqrt{\kappa} \int_{0}^{t} U(\kappa(t-s)) a(0) d B_{s}$. To prove the conclusion, since $\left\{Y_{t}^{(k)}, t>0\right\}$ is a Gaussian system with mean 0 , we may only show that the covariance function of $\left\{Y_{t}^{(s)}\right\}$ converges to that of $\left\{Y_{t}\right\}$.

For $0<t_{1} \leqq t_{2}<\infty$ and $\psi_{1}, \psi_{2} \in H$,

$$
\begin{aligned}
& E\left[\left\langle Y_{t_{1}}^{(\kappa)}, \psi_{1}\right\rangle\left\langle Y_{t_{2}}^{(\kappa)}, \psi_{2}\right\rangle\right] \\
& =\kappa \int_{0}^{t_{1}}\left\langle a^{*}(0) U\left(\kappa\left(t_{1}-s\right)\right) \psi_{1}, a^{*}(0) U\left(\kappa\left(t_{2}-s\right)\right) \psi_{2}\right\rangle d s \\
& =\sum_{j, k=1}^{\infty}\left(\lambda_{j}+\lambda_{k}\right)^{-1}\left\{e^{-\kappa \lambda_{k}\left(t_{2}-t_{1}\right)}-e^{-\kappa\left(\lambda_{j} t_{1}+\lambda_{k} t_{2}\right)}\right\} \\
& \quad \times\left\langle a^{*}(0) \phi_{j}, a^{*}(0) \phi_{k}\right\rangle\left\langle\psi_{1}, \phi_{j}\right\rangle\left\langle\psi_{2}, \phi_{k}\right\rangle .
\end{aligned}
$$

As $\kappa \rightarrow \infty$, the right hand side of this equality converges to 0 when $t_{1} \neq t_{2}$ and to $\left\|\psi_{1}\right\|^{2}$ when $t_{1}=t_{2}$ and $\psi_{1}=\psi_{2}$. We therefore have the conclusion.

(Q.E.D.)

Case 3. We shall show that the process $\kappa X_{t}^{(\varepsilon)}$ converges to the constant vector $A^{-1} b(0)$ as $\kappa \rightarrow \infty$. To prove this result, we prepare the following lemma. 
Lemma 6.6. The norm $\left\|\kappa X_{t, 2}^{(x)}\right\|$ converges to 0 uniformly in $t$ on each compact subset of $(0, \infty)$ as $\kappa(\in N) \rightarrow \infty$ (a.s.).

Proof. In the case 3, Lemma 6.4 holds by taking $n=0$. Therefore, we have the following two inequalities.

(6.11) For each $\varepsilon>0$,

$$
P\left(\sup _{t \in[0, T]}\left\|X_{t, 2}^{(\kappa)}\right\|>\kappa^{-1 / 2+\varepsilon}\right) \leqq C e^{-\tilde{C}_{r}^{2 \varepsilon}}, \quad \text { for sufficiently large } \kappa,
$$

and

$$
\sup _{t \in[0, T]}\left\|X_{t, 3}^{(\kappa)}\right\| \leqq K /(\lambda \kappa) \quad \text { (a.s.) . }
$$

Combining these two estimates with the following one on $X_{t, 1}^{(\kappa)}$ :

$$
\left\|X_{t, 1}^{(\kappa)}\right\| \leqq e^{-\lambda \kappa t}\left\|X_{0}\right\|
$$

we get

$$
P\left(\sup _{t \in\left[\kappa^{-1 / 2}, T\right]}\left\|X_{t}^{(\kappa)}\right\|>2 \kappa^{-1 / 2+\varepsilon}\right) \leqq C e^{-\tilde{c} k^{2 \varepsilon}}, \quad \text { for sufficiently large } \kappa .
$$

By this estimate and the equality:

$$
X_{t, 2}^{(\kappa)}=U\left(\kappa\left(t-t_{1}\right)\right) X_{t_{1}, 2}^{(\kappa)}+\tilde{X}_{t-t_{1}, t_{1}}^{(\kappa)}, \quad\left(t>t_{1}>0\right),
$$

where

$$
\tilde{X}_{t, t_{1}}^{(\kappa)}=\int_{0}^{t} U(\kappa(t-s)) a\left(X_{s+t_{1}}^{(\kappa)}\right) d\left(B_{s+t_{1}}-B_{t_{1}}\right)
$$

we have

$$
\begin{aligned}
& P\left(\sup _{t \in[2 \kappa-1 / 2, T]}\left\|X_{t, 2}^{(\kappa)}\right\|>\kappa^{-1+2 \varepsilon}\right) \\
& \leqq P\left(\left\|U(\sqrt{\kappa}) X_{\kappa^{-1 / 2}, 2}^{(\kappa)}\right\|>\kappa^{-1+2 \varepsilon} / 2\right)+P\left(\sup _{t \in\left[2 \kappa^{-1 / 2}, T\right]}\left\|\tilde{X}_{t-\kappa^{-1 / 2, \kappa^{-1 / 2}}}^{(\kappa)}\right\|>\kappa^{-1+2 \varepsilon} / 2\right) \\
& \leqq P\left(\left\|X_{\kappa^{-1 / 2}, 2}^{(\kappa)}\right\|>\kappa^{-1+2 \varepsilon} e^{\lambda \sqrt{\kappa}} / 2\right)+P\left(\sup _{t \in\left[\kappa^{-1 / 2}, T\right]}\left\|X_{t}^{(\kappa)}\right\|>2 \kappa^{-1 / 2+\varepsilon}\right) \\
& +P\left(\sup _{t \in\left[0, T-\varepsilon^{-1 / 2}\right]}\left\|\tilde{X}_{t, \kappa^{-1 / 2}}^{(\kappa)}\right\|>\kappa^{-1+2 \varepsilon} / 2, \sup _{t \in\left[\varepsilon^{-1 / 2}, T\right]}\left\|X_{t}^{(\kappa)}\right\| \leqq 2 \kappa^{-1 / 2+\varepsilon}\right) \\
& \leqq\left(2 \kappa^{1-2 \varepsilon} e^{-\lambda \sqrt{\kappa}}\right)^{2} C\left(e^{-2 \lambda \sqrt{\kappa}}+\kappa^{-1}\right)+C e^{-\tilde{C}^{2} \kappa^{2 \varepsilon}} \\
& +\sum_{k=1}^{\infty} K_{1} \exp \left\{-C_{1} \lambda_{k} \kappa^{2 \varepsilon} /\left(48 K^{2} k^{1+\varepsilon}\right)\right\}\left(\lambda_{k} \kappa T+1\right) \\
& \leqq C_{2} \exp \left(-\tilde{C}_{2} \kappa^{2 \varepsilon}\right), \quad \text { for sufficiently large } \kappa \text {. }
\end{aligned}
$$


Repeating a similar procedure once more, we can show that

$$
\begin{gathered}
P\left(\sup _{t \in\left[3 \kappa^{-1 / 2}, T\right]}\left\|X_{t, 2}^{(\kappa)}\right\|>\kappa^{-3 / 2+3 \varepsilon}\right) \leqq C_{3} \exp \left(-C_{3} \kappa^{2 \varepsilon}\right), \\
\text { for sufficiently large } \kappa .
\end{gathered}
$$

Taking $\varepsilon$ such that $0<\varepsilon<1 / 6$, the Borel-Cantelli's lemma proves that

$$
P\left(\sup _{t \in[3 \kappa-1 / 2, T]}\left\|\kappa X_{t, 2}^{(k)}\right\|<\kappa^{-1 / 2+3 \varepsilon} \quad \text { as } \quad \kappa(\in N) \longrightarrow \infty\right)=1 .
$$

This implies the conclusion.

(Q.E.D.)

Theorem 6.3. In the case $3, \kappa X_{t}^{(\kappa)}$ tends to $A^{-1} b(0)$ in the following sense:

$$
\begin{aligned}
& P\left(\lim _{\kappa(\in N) \rightarrow \infty}\left\|\kappa X_{t}^{(\kappa)}-A^{-1} b(0)\right\|\right.=0 \text { uniformly in } t \text { on } \\
&\text { each compact subset of }(0, \infty))=1 .
\end{aligned}
$$

Proof. The estimates (6.11)-(6.13) imply that

$$
P\left(\left\|X_{t}^{(\kappa)}\right\| \leqq C\left(e^{-\lambda \kappa t}+\kappa^{-1 / 2+\varepsilon}\right) \quad \text { for every } t \in[0, T], \quad \kappa(\in N) \longrightarrow \infty\right)=1 .
$$

Therefore, we have

$$
\begin{aligned}
\| \kappa X_{t, 3}^{(\kappa)}- & A^{-1} b(0) \|^{2} \\
\leqq & 2\left\|\kappa X_{t, 3}^{(\kappa)}-\kappa \int_{0}^{t} U(\kappa(t-s)) b(0) d s\right\|^{2} \\
& +2\left\|\kappa \int_{0}^{t} U(\kappa(t-s)) b(0) d s-A^{-1} b(0)\right\|^{2} \\
\leqq & 2\left(\kappa \int_{0}^{t} e^{-\lambda \kappa(t-s)} K\left\|X_{s}^{(\kappa)}\right\| d s\right)^{2} \\
& \quad+2\left\|\sum_{k=1}^{\infty}\left\{\int_{0}^{t} \kappa e^{-\kappa \lambda_{k} s} d s-\lambda_{k}^{-1}\right\}\left\langle b(0), \phi_{k}\right\rangle \phi_{k}\right\|^{2} \\
\leqq & 2 K^{2} C^{2} \kappa^{2}\left(e^{-\lambda \kappa t} t+\kappa^{-1 / 2+s}(\lambda \kappa)^{-1}\right)^{2}+2 \sum_{k=1}^{\infty} \lambda_{k}^{-2} e^{-2 \kappa \lambda_{k} t}\left\langle b(0), \phi_{k}\right\rangle^{2} \\
& \longrightarrow 0 \text { uniformly in } t \text { on each compact subset of }(0, \infty)
\end{aligned}
$$

Noting Lemma 6.6, we have the conclusion.

Case 4. In this case, we further assume the following condition.

(A.5) The coefficients $a$ and $b$ are Fréchet differentiable at 0 . 
Consider the following stochastic differential equation:

$$
d Y_{t}=P_{r} a^{\prime}(0)\left(Y_{t}\right) d B_{t}+P_{r} b^{\prime}(0)\left(Y_{t}\right) d t, \quad Y_{0}=P_{r} X_{0},
$$

where $a^{\prime}(0)(\cdot)$ and $b^{\prime}(0)(\cdot)$ are Fréchet derivatives at 0 of $a$ and $b$, respectively.

LEMma 6.7. The equation (6.14) has a unique solution $Y_{t}$ which belongs to $C\left([0, \infty), P_{r} H\right)$ (a.s.).

Proof. The coefficients $P_{r} a^{\prime}(0)$ and $P_{r} b^{\prime}(0)$ are Lipschitz continuous since, for $Y_{1}, Y_{2} \in H$, we get

$$
\begin{aligned}
& \left\|P_{r} a^{\prime}(0)\left(Y_{1}\right)-P_{r} a^{\prime}(0)\left(Y_{2}\right)\right\|_{2}^{2} \\
& \quad=\sum_{k=1}^{n}\left\|\left\{a^{\prime}(0)\left(Y_{1}\right)-a^{\prime}(0)\left(Y_{2}\right)\right\}^{*} \phi_{k}\right\|^{2} \\
& \quad=\sum_{k=1}^{n}\left\|\lim _{\varepsilon \downarrow 0} \frac{1}{\varepsilon}\left(a^{*}\left(\varepsilon Y_{1}\right)-a^{*}\left(\varepsilon Y_{2}\right)\right) \phi_{k}\right\|^{2} \leqq n K^{2}\left\|Y_{1}-Y_{2}\right\|^{2},
\end{aligned}
$$

and

$$
\begin{aligned}
& \left\|P_{r} b^{\prime}(0)\left(Y_{1}\right)-P_{r} b^{\prime}(0)\left(Y_{2}\right)\right\| \\
& \quad \leqq\left\|\lim _{\varepsilon \downarrow 0} \frac{1}{\varepsilon}\left(b\left(\varepsilon Y_{1}\right)-b\left(\varepsilon Y_{2}\right)\right)\right\| \leqq K\left\|Y_{1}-Y_{2}\right\| .
\end{aligned}
$$

We therefore have the conclusion in a similar way to the proof of Lemma 6.1 .

Setting $Y_{t}^{(\kappa)}=e^{\lambda \kappa} X_{t}^{(\kappa)}$ and $Y_{t, i}^{(\kappa)}=e^{\lambda \kappa t} X_{t, i}^{(\kappa)}(i=1,2,3)$, we have the following estimate.

LEMma 6.8. $\sup _{(t, \kappa) \in[0, T] \times[1, \infty)} E\left[\left\|Y_{t}^{(\kappa)}\right\|^{2}\right]<\infty$.

Proof. By (6.5), we have

$$
\begin{aligned}
E\left[\left\|Y_{t}^{(\kappa)}\right\|^{2}\right] \leqq & 3\left\|e^{\lambda \kappa t} U(\kappa t) X_{0}\right\|^{2}+3 E\left[\left\|\int_{0}^{t} e^{\lambda \kappa t} U(\kappa(t-s)) a\left(e^{-\lambda \kappa s} Y_{s}^{(\kappa)}\right) d B_{s}\right\|^{2}\right] \\
& +3 E\left[\left\|\int_{0}^{t} e^{\lambda \kappa t} U(\kappa(t-s)) b\left(e^{-\lambda \kappa s} Y_{s}^{(\kappa)}\right) d s\right\|^{2}\right] \\
\leqq & 3\left\|X_{0}\right\|^{2}+3 K^{2} \int_{0}^{t} \sum_{k=1}^{\infty} e^{-2 \kappa(\lambda k-\lambda)(t-s)} E\left[\left\|Y_{s}^{(\kappa)}\right\|^{2}\right] d s \\
& +3 K^{2} t \int_{0}^{t} E\left[\left\|Y_{s}^{(\kappa)}\right\|^{2}\right] d s \\
\leqq & C_{1}+C_{2} \int_{0}^{t}(t-s)^{-\alpha} E\left[\left\|Y_{s}^{(\kappa)}\right\|^{2}\right] d s, \quad \alpha=(1+\delta)^{-1}<1 .
\end{aligned}
$$

This implies the desired estimate.

(Q.E.D.) 
The asymptotic behavior of the process $Y_{t}^{(n)}$ is given by the following theorem.

TheOREM 6.4. In the case 4,

$$
E\left[\left\|Y_{t}^{(\kappa)}-Y_{t}\right\|^{2}\right] \longrightarrow 0
$$

uniformly in $t$ on each compact subset of $(0, \infty)$ as $\kappa \rightarrow \infty$.

Proof. Set $I^{(\kappa)}(t)=E\left[\left\|Y_{t}^{(\kappa)}-Y_{t}\right\|^{2}\right]$ and $I_{i}^{(\kappa)}(t)=E\left[\left\|Y_{t, i}^{(\kappa)}-Y_{t, i}\right\|^{2}\right](i=$ 1, 2,3). As for the $I_{1}^{(\kappa)}(t)$,

$$
I_{1}^{(\kappa)}(t) \leqq \exp \left\{-2\left(\lambda_{n+1}-\lambda\right) \kappa t\right\}\left\|X_{0}\right\|^{2}
$$

Next, we have

$$
\begin{aligned}
I_{2}^{(\kappa)}(t) \leqq & 2 E\left[\left\|\int_{0}^{t}\left\{e^{\lambda \kappa t} U(\kappa(t-s)) a\left(X_{s}^{(\kappa)}\right)-e^{\lambda \kappa s} P_{r} a\left(X_{s}^{(\kappa)}\right)\right\} d B_{s}\right\|^{2}\right] \\
& +2 E\left[\left\|\int_{0}^{t} P_{r}\left\{e^{\lambda \kappa s} a\left(X_{s}^{(\kappa)}\right)-a^{\prime}(0)\left(Y_{s}\right)\right\} d B_{s}\right\|^{2}\right] \\
\leqq & 2 K^{2} M \int_{0}^{t} \sum_{k=n+1}^{\infty} e^{-2\left(\lambda_{k}-\lambda\right) \kappa(t-s)} d s \\
& +2 E\left[\int_{0}^{t} \sum_{k=1}^{\infty}\left\|\left\{e^{\lambda \kappa s} a^{*}\left(e^{-\lambda \kappa s} Y_{s}^{(\kappa)}\right)-a^{\prime}(0)^{*}\left(Y_{s}\right)\right\} P_{r} \phi_{k}\right\|^{2} d s\right] \\
\leqq & f(\kappa)+4 n K^{2} \int_{0}^{t} E\left[\left\|Y_{s}^{(\kappa)}-Y_{s}\right\|^{2}\right] d s, \quad t \leqq T,
\end{aligned}
$$

where

$$
\begin{aligned}
f(\kappa)= & \sum_{k=n+1}^{\infty} K^{2} M\left\{\left(\lambda_{k}-\lambda\right) \kappa\right\}^{-1} \\
& +\sum_{k=1}^{n} \int_{0}^{T} E\left[\left\|\left\{e^{\lambda \kappa s} a^{*}\left(e^{-\lambda \kappa s} Y_{s}\right)-a^{\prime}(0)^{*}\left(Y_{s}\right)\right\} \phi_{k}\right\|^{2}\right] d s .
\end{aligned}
$$

A bound on $I_{3}^{(\kappa)}(t)$ is similar to $I_{2}^{(\kappa)}(t)$, so that, summing up these estimates, we have

$$
I^{(\kappa)}(t) \leqq C e^{-\tilde{c} x t}+\tilde{f}(\kappa)+C \int_{0}^{t} I^{(x)}(s) d s, \quad t \leqq T,
$$

where the function $\tilde{f}(\kappa)$ behaves as $\tilde{f}(\kappa) \rightarrow 0$ (as $\kappa \rightarrow \infty$ ). Gronwall's lemma implies that

$$
\begin{aligned}
I^{(\kappa)}(t) & \leqq C e^{-\tilde{C} \kappa t}+\tilde{f}(\kappa)+C \int_{0}^{t} e^{C(t-s)}\left(C e^{-\tilde{C}_{\kappa} s}+\tilde{f}(\kappa)\right) d s \\
& \leqq C e^{-C_{\kappa} t}+\tilde{f}(\kappa)+C^{2} e^{C t}(\tilde{C} \kappa)^{-1}+\tilde{f}(\kappa) e^{C t}, \quad t \leqq T,
\end{aligned}
$$

which proves the conclusion.

(Q.E.D.) 


\section{Appendix I}

Here, we investigate the joint-continuity of solutions of stochastic partial differential equations (SPDE).

(a) Known results on partial differential equations.

Let $G$ be a bounded region in $R^{n}$ surrounded by a smooth hypersurface $\partial G$. We assume that $G$ has a restricted cone property (e.g. S. Agmon [1]). Let $A$ be a closed operator on $L^{2}(G)$ determined by the following conditions (i), (ii) and satisfying the condition (iii).

(i) The domain of $A$ is given by

$$
\mathscr{D}(A)=\left\{\phi \in H^{2 m}(G) ; \beta_{j} \phi=0 \text { on } \partial G, 1 \leqq j \leqq m\right\},
$$

where $H^{2 m}(G)$ is the Sobolev space of order $2 m$ and $\left\{\beta_{j}\right\}_{j=1}^{m}$ is a normal system of boundary operators (J. L. Lions and E. Magenes [14]).

(ii) $A$ is a uniformly elliptic differential operator of order $2 m(m \in N)$ :

$$
A \phi(\sigma)=\sum_{|\nu|=0}^{2 m} \alpha_{\nu}(\sigma) D^{\nu} \phi(\sigma), \quad \phi \in \mathscr{D}(A),
$$

where $\nu=\left(\nu_{1}, \cdots, \nu_{n}\right),|\nu|=\nu_{1}+\cdots+\nu_{n}$,

$$
D^{\nu}=\frac{\partial^{|\nu|}}{\left(\partial \sigma^{1}\right)^{\nu_{1}} \cdots\left(\partial \sigma^{n}\right)^{\nu}}, \quad \sigma=\left(\sigma^{i}\right)_{i=1}^{n} \quad \text { and } \quad \alpha_{\nu} \in C_{b}^{\infty}(G) \text {. }
$$

(iii) The operator $A$ is self-adjoint and non-negative.

Under these conditions, the operator $A$ has pure point spectrum $\left\{\lambda_{k}\right\}_{k=1}^{\infty}$ such that $\lambda_{k} \sim c k^{2 m / n}(c>0)$ as $k \rightarrow \infty$. The semi-group $U(t)$ on $L^{2}(G)$ generated by $-A$ is an integral operator with a symmetric kernel $p(t, \sigma, \tau)$ $\in L^{2}(G \times G)$ for each $t>0$. The following estimate on $p$ was given by $\mathrm{R}$. Arima [2].

$$
\begin{gathered}
\left|\frac{\partial}{\partial \tau^{i}} p(t, \sigma, \tau)\right| \leqq C t^{-(n+1) /(2 m)} \exp \left\{-\tilde{C}\left(|\sigma-\tau|^{2 m} / t\right)^{1 /(2 m-1)}\right\} \\
(t, \sigma, \tau) \in(0, T) \times \bar{G} \times G, \quad 1 \leqq i \leqq n \text { with } \quad \text { C, } \tilde{C}>0 .
\end{gathered}
$$

(b) SPDE with non-homogeneous boundary conditions.

Let $A^{i}(1 \leqq i \leqq d)$ be operators on $L^{2}(G)$ which satisfy the conditions (i)-(iii) with the normal systems $\left\{\beta_{j}^{i}\right\}_{j=1}^{m}(1 \leqq i \leqq d)$ of boundary operators. We define an operator $A$ on $H=L^{2}\left(G, R^{d}\right)$ by

$$
A X=\left(A^{i} X^{i}\right)_{i=1}^{d} \quad \text { for } X=\left(X^{i}\right)_{i=1}^{d} \in H=\left(L^{2}(G)\right)^{d} .
$$


We assume that $2 m$ is larger than $n$. Then, the operator $A$ satisfies the condition (A.1) in $\S 1$.

Assuming that $a$ and $b$ satisfy the conditions (A.2) and (A.3) in $\S 1$ with $H=L^{2}\left(G, R^{d}\right)$, respectively, we consider the following initial-boundary value problem for given initial state $X(0)=X(0, \cdot) \in H$ and $R^{d}$-valued boundary functions $f_{j}(1 \leqq j \leqq m)$.

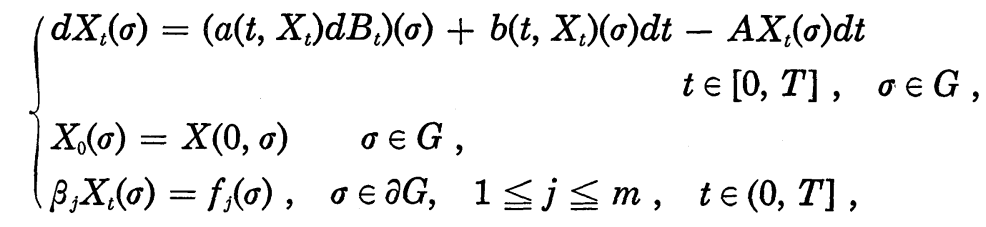

where $B_{t}$ is a cylindrical Brownian motion on $H$ and where $\beta_{j} X$ is defined by

$$
\beta_{j} X=\left(\beta_{j}^{i} X^{i}\right)_{i=1}^{d} \quad \text { for } X=\left(X^{i}\right)_{i=1}^{d} .
$$

An $H$-valued $\mathscr{F}_{t}$-adapted process $X_{t}$ is called a solution of the equation (AI.2), if it satisfies the following integral equation:

$$
\begin{aligned}
X_{t} & =X_{t, 1}+\int_{0}^{t} U(t-s) a\left(s, X_{s}\right) d B_{s}+\int_{0}^{t} U(t-s) b\left(s, X_{s}\right) d s \\
& \equiv X_{t, 1}+X_{t, 2}+X_{t, 3} .
\end{aligned}
$$

In this equation, $\{U(t), t \geqq 0\}$ is a semi-group on $H$ generated by $-A$ and $X_{t, 1}$ is the solution of the following partial differential equation:

$$
\left\{\begin{array}{l}
\frac{\partial X_{t, 1}}{\partial t}(\sigma)=-A X_{t, 1}(\sigma), \quad \sigma \in G, \quad t \in[0, T] \\
X_{0,1}(\sigma)=X(0, \sigma), \quad \sigma \in G, \\
\beta_{j} X_{t, 1}(\sigma)=f_{j}(\sigma), \quad \sigma \in \partial G, \quad 1 \leqq j \leqq m, \quad t \in(0, T] .
\end{array}\right.
$$

We assume the conditions on the functions $\left\{f_{j}\right\}_{j=1}^{m}$ and $X(0, \cdot)$ to guarantee that the equation (AI.4) has a unique solution $X_{t, 1}$ which belongs to $C([0, T], H)$ (these conditions can be found, e.g., in R. Arima [2]). Then, we can show the following existence and uniqueness theorem of the solution of the SPDE (AI.2).

Lemma AI.1. There exists a unique solution $X_{t}$ of the SPDE (AI.2) such that $\sup _{t \in[0, T]} E\left[\left\|X_{t}\right\|^{2}\right]<\infty$ for each $T<\infty$ and $X_{t} \in C([0, T], H)$ (a.s.).

Proof. Denote by $Z_{t}$ the solution of the equation (AI.4) with $X(0, \cdot)$ $\equiv 0$. Since $\tilde{a}(t, X)=a\left(t, X+Z_{t}\right)$ and $\tilde{b}(t, X)=b\left(t, X+Z_{t}\right)$ satisfy the 
conditions (A.2) and (A.3) in $\S 1$, respectively, by Theorem 1.1, the following SPDE with homogeneous boundary conditions has a unique solution $Y_{t}$.

$$
\left\{\begin{array}{l}
d Y_{t}=\tilde{a}\left(t, Y_{t}\right) d B_{t}+\tilde{b}\left(t, Y_{t}\right) d t-A Y_{t} d t \\
Y_{0}=X(0) \\
\beta_{j} Y_{t}(\sigma)=0, \quad \sigma \in \partial G .
\end{array}\right.
$$

We can easily show that $X_{t}=Y_{t}+Z_{t}$ is the unique solution of the SPDE (AI.2) such that $\sup _{t \in[0, T]} E\left[\left\|X_{t}\right\|^{2}\right]<\infty$ for each $T<\infty$ and $X_{t} \in C([0, T], H)$ (a.s.).

(c) Joint-continuity of the solution $X_{t}(\sigma)$ of SPDE (AI.2).

Here, we assume that $a$ is bounded, i.e.,

$$
\|a(t, X)\|_{\mathscr{L}(H)}<K \quad \text { for every }(t, X) \in[0, T] \times H .
$$

Under this assumption, we can show the following lemma by noting that

$$
\int_{0}^{T}\|U(t)\|_{2}^{2} d s<\infty
$$

and

$$
X_{t, 1} \in C([0, T], H) .
$$

LEMma AI.2. For each $p>0$,

$$
\sup _{t \in[0, T]} E\left[\left\|X_{t}\right\|^{p}\right]<\infty .
$$

Concerning the joint-continuity in $(t, \sigma)$ of the solution $X_{t}(\sigma)$ of the SPDE (AI.2), we have the following result.

Proposition AI.1. (i) The terms $X_{t, 2}(\sigma)$ and $X_{t, 3}(\sigma)$ in (AI.3) have versions jointly continuous in $(t, \sigma) \in[0, T] \times \bar{G}$. (ii) If $X_{t, 1}(\sigma)$ belongs to $C\left([0, T] \times \bar{G}, R^{d}\right)$, then the solution $X_{t}(\sigma)$ of the SPDE (AI.2) is jointly continuous in $(t, \sigma) \in[0, T] \times \bar{G}$.

Proof. Denote by $p_{i}(t, \sigma, \tau)(1 \leqq i \leqq d, t \in[0, T], \sigma, \tau \in \bar{G})$ the integral kernel of the semi-group $U_{i}(t)$ on $L^{2}(G)$ generated by $-A^{i}$. We shall adopt a description in component-wise, e.g.,

$$
X_{t, 2}=\left(X_{t, 2}^{i}\right)_{i=1}^{d}, \quad B_{t}=\left(B_{t}^{i}\right)_{i=1}^{d} \quad \text { and } \quad a(t, X)=\left(a_{i j}(t, X)\right)_{i, j=1}^{d}
$$

with $a_{i j}(t, X) \in \mathscr{L}\left(L^{2}(G)\right)$. Then, noting that 


$$
\begin{aligned}
X_{t, 2}^{i}(\sigma) & =\sum_{j=1}^{d} \int_{0}^{t} \int_{G} p_{i}(t-s, \sigma, \tau)\left(a_{i j}\left(s, X_{s}\right) d B_{s}^{j}\right)(\tau) d \tau \\
& =\sum_{j=1}^{d} \int_{0}^{t} \int_{G}\left(a_{i j}^{*}\left(s, X_{s}\right) p_{i}(t-s, \sigma, \cdot)\right)(\tau) d B_{s}^{j}(\tau) d \tau,
\end{aligned}
$$

we have

$$
\begin{gathered}
E\left[\left|X_{t_{1}, 2}^{i}\left(\sigma_{1}\right)-X_{t_{2}, 2}^{i}\left(\sigma_{2}\right)\right|^{2 r}\right] \\
\leqq C_{1} \sum_{j=1}^{d}\left[\int _ { 0 } ^ { t _ { 2 } } E \left[\left\{\int _ { G } \left(a _ { i j } ^ { * } ( s , X _ { s } ) \left\{p_{i}\left(t_{1}-s, \sigma_{1}, \cdot\right) \chi_{\left\{s \leqq t_{1}\right\}}\right.\right.\right.\right.\right. \\
\left.\left.\left.\left.\left.-p_{i}\left(t_{2}-s, \sigma_{2}, \cdot\right)\right\}\right)^{2}(\tau) d \tau\right\}^{r}\right]^{1 / r} d s\right]^{r} \\
\leqq C_{2}\left\{\int_{0}^{t_{1}} p_{i}\left(2 s, \sigma_{1}, \sigma_{1}\right) d s+\int_{0}^{t_{2}} p_{i}\left(2 s, \sigma_{2}, \sigma_{2}\right) d s\right. \\
\left.\quad-2 \int_{\left(t_{2}-t_{1}\right) / 2}^{\left(t_{1}+t_{2}\right) / 2} p_{i}\left(2 s, \sigma_{1}, \sigma_{2}\right) d s\right\}^{r} \\
\quad \text { for } 0 \leqq t_{1} \leqq t_{2} \leqq T, \quad \sigma_{1}, \sigma_{2} \in \bar{G} \text { and } r \in N .
\end{gathered}
$$

To bound the right hand side of the above estimation, we set

$$
I^{j}=\left|\int_{0}^{t_{j}} p_{i}\left(2 s, \sigma_{1}, \sigma_{1}\right) d s-\int_{\left(t_{2}-t_{1}\right) / 2}^{\left(t_{1}+t_{2}\right) / 2} p_{i}\left(2 s, \sigma_{1}, \sigma_{2}\right) d s\right|, \quad j=1,2 .
$$

Then, we have

$$
\begin{aligned}
I^{1} \leqq & \left|\int_{0}^{t_{1}}\left\{p_{i}\left(2 s, \sigma_{1}, \sigma_{1}\right)-p_{i}\left(2 s, \sigma_{1}, \sigma_{2}\right)\right\} d s\right| \\
& +\left|\int_{0}^{\left(t_{2}-t_{1}\right) / 2} p_{i}\left(2 s, \sigma_{1}, \sigma_{2}\right) d s\right|+\left|\int_{t_{1}}^{\left(t_{1}+t_{2}\right) / 2} p_{i}\left(2 s, \sigma_{1}, \sigma_{2}\right) d s\right| \\
= & I_{1}^{1}+I_{2}^{1}+I_{3}^{1} .
\end{aligned}
$$

The first term $I_{1}^{1}$ can be estimated as follows.

$$
\begin{aligned}
I_{1}^{1} & \leqq \int_{0}^{t_{1}}\left|\int_{0}^{1} \sum_{j=1}^{d} \frac{\partial}{\partial \tau^{j}} p_{i}\left(2 s, \sigma_{1}, \sigma_{1}+\alpha\left(\sigma_{2}-\sigma_{1}\right)\right) \times\left(\sigma_{2}^{j}-\sigma_{1}^{j}\right) d \alpha\right| d s \\
& \leqq \int_{0}^{t_{1}} C_{3}\left|\sigma_{2}-\sigma_{1}\right| d s \int_{0}^{1} s^{-(n+1) /(2 m)} \exp \left\{-\tilde{C}\left(\left|\alpha\left(\sigma_{2}-\sigma_{1}\right)\right|^{2 m} / s\right)^{1 /(2 m-1)}\right\} d \alpha \\
& \leqq C_{4}\left|\sigma_{2}-\sigma_{1}\right|^{1 / p} \int_{0}^{t_{1}} s^{-(n+1) /(2 m)+1 /(2 m q)} d s,
\end{aligned}
$$

where $1 / p+1 / q=1, p, q>1$. Taking $q$ such that $(n+1) / 2 m-1 / 2 m q$ $<1$, we have

$$
I_{1}^{1} \leqq C_{5}\left|\sigma_{1}-\sigma_{2}\right|^{1 / p}
$$

As for the second term $I_{2}^{1}$, 


$$
{ }^{I} I_{2}^{1} \leqq C_{6} \int_{0}^{\left(t_{2}-t_{1}\right) / 2} s^{-n /(2 m)} d s \leqq C_{7}\left|t_{1}-t_{2}\right|^{1-n /(2 m)} .
$$

Since the third term $I_{3}^{1}$ can be estimated in a similar way to $I_{2}^{1}$, we have the following bound on $I^{1}$ :

$$
I^{1} \leqq C_{8}\left\{\left|\sigma_{1}-\sigma_{2}\right|^{1 / p}+\left|t_{1}-t_{2}\right|^{1-n /(2 m)}\right\} \text {. }
$$

The term $I^{2}$ can be estimated in a similar way to $I^{1}$. Hence, we have

$$
\begin{aligned}
& E\left[\left|X_{t_{1}, 2}^{i}\left(\sigma_{1}\right)-X_{t_{2}, 2}^{i}\left(\sigma_{2}\right)\right|^{2 r}\right] \\
& \quad \leqq C\left\{\left|\sigma_{1}-\sigma_{2}\right|^{1 / p}+\left|t_{1}-t_{2}\right|^{1-n /(2 m)}\right\}^{r} .
\end{aligned}
$$

Taking $r$ sufficiently large, Kolmogorov's regularization theorem (Proposition 1.2) implies that $X_{t, 2}^{i}(\sigma)$ belongs to $C([0, T] \times \bar{G})$ (a.s.). Therefore, $X_{t, 2}(\sigma)$ belongs to $C\left([0, T] \times \bar{G}, R^{d}\right)$ (a.s.). Using Lemma AI.2, we can also show that $X_{t, 3}(\sigma)$ belongs to $C\left([0, T] \times \bar{G}, R^{d}\right)$ (a.s.). We therefore have the assertion (i). The assertion (ii) follows from (i) immediately. (Q.E.D.)

Remark AI.1. Under the condition (2.2) on the initial state $X_{0}$, the system (2.8) of heat equations has a jointly continuous solution $X_{t, 1}(\sigma)$. Therefore, the equation (2.5) of the string has a jointly continuous solution $X_{t}(\sigma)$ in $(t, \sigma) \in[0, \infty) \times[0,1]$.

(d) The strong Markov property of the solution of the equation (2.5).

Similarly to the finite dimensional case (e.g. H. P. McKean Jr. [16]), the solution $X_{t}(\sigma)$ of the equation (2.5) has the strong Markov property in the following sense.

Proposition AI.2. Assume that $Y \in \mathscr{C}$ satisfies the condition (2.2). If $\boldsymbol{t}$ is an $\left(\mathscr{F}_{t}\right)$-stopping time, then, conditional on $t<\infty$ and $X_{t}=Y$, the future $X_{t}^{+}=X(t+t): t>0$ is independent of $\mathscr{F}_{t}$ and identical in law to the solution of the equation (2.5) with $X_{0}=Y$.

\section{Appendix II}

We summarize several facts about the fundamental solutions $q^{(N)}(t, k / N, l / N)$ and $q(t, \sigma, \tau)$ of $(1 / 2) \Delta^{(N)}$ and $(1 / 2) \Delta$, respectively.

(a) The fundamental solutions $q^{(N)}(t, k / N, l / N)$ and $q(t, \sigma, \tau)$ have the following representations, respectively.

(AII. 1)

$$
q^{(N)}\left(t, \frac{k}{N}, \frac{\ell}{N}\right)=\sum_{i=1}^{N} \xi_{i}^{(N)}\left(\frac{k}{N}\right) \xi_{i}^{(N)}\left(\frac{\ell}{N}\right) e^{-N^{2 \lambda_{i}^{(N)} t}},
$$


where

$$
\begin{aligned}
& \xi_{i}^{(N)}\left(\frac{k}{N}\right)=\left\{\begin{array}{lc}
1 & i=1 \\
\left(\lambda_{i}^{(N)}\right)^{-1 / 2}\left\{\sin \frac{\pi(i-1) k}{N}-\sin \frac{\pi(i-1)(k-1)}{N}\right\}, & 2 \leqq i \leqq N,
\end{array}\right. \\
& \lambda_{i}^{(N)}=1-\cos \frac{\pi(i-1)}{N}, \quad 1 \leqq i \leqq N, \quad \text { in the case }(\mathrm{I}) \text {, }
\end{aligned}
$$

and

$$
\begin{aligned}
& \xi_{i}^{(N)}\left(\frac{k}{N}\right)=\left(\frac{2 N+1}{2 N} \lambda_{i}^{(N)}\right)^{-1 / 2}\left\{\cos \frac{\pi(i-1 / 2)(2 k+1)}{2 N+1}\right. \\
& \left.-\cos \frac{\pi(i-1 / 2)(2 k-1)}{2 N+1}\right\}, \\
& \lambda_{i}^{(N)}=1-\cos \frac{\pi(2 i-1)}{2 N+1}, \quad 1 \leqq i \leqq N, \text { in the case (II) },
\end{aligned}
$$

and

$$
\begin{aligned}
& \xi_{i}^{(N)}\left(\frac{k}{N}\right)=\left(\frac{N+1}{N} \lambda_{i}^{(N)}\right)^{-1 / 2}\left\{\cos \frac{\pi i(2 k+1)}{2(N+1)}-\cos \frac{\pi i(2 k-1)}{2(N+1)}\right\}, \\
& \lambda_{i}^{(N)}=1-\cos \frac{\pi i}{N+1}, \quad 1 \leqq i \leqq N, \text { in the case (III) } .
\end{aligned}
$$

While

(AII. 2)

$$
q(t, \sigma, \tau)=\sum_{i=1}^{\infty} \xi_{i}(\sigma) \xi_{i}(\tau) e^{-\pi^{2} \lambda_{i} t / 2}
$$

where

$$
\begin{aligned}
& \xi_{i}(\sigma)= \begin{cases}1 & i=1, \\
\sqrt{2} \cos \pi(i-1) \sigma, & 2 \leqq i<\infty,\end{cases} \\
& \lambda_{i}=(i-1)^{2}, \quad 1 \leqq i<\infty, \quad \text { in the case }(\mathrm{I}),
\end{aligned}
$$

and

$$
\begin{aligned}
& \xi_{i}(\sigma)=\sqrt{2} \sin \pi(i-1 / 2) \sigma, \\
& \lambda_{i}=(i-1 / 2)^{2}, \quad 1 \leqq i<\infty, \text { in the case (II), }
\end{aligned}
$$

and

$$
\begin{aligned}
& \xi_{i}(\sigma)=\sqrt{2} \sin \pi i \sigma, \\
& \lambda_{i}=i^{2}, \quad 1 \leqq i<\infty, \quad \text { in the case (III) } .
\end{aligned}
$$

We note that the right hand side of (AII.2) is a uniform convergent sum 
in $(t, \sigma, \tau)$ on each compact subset of $(0, \infty) \times[0,1] \times[0,1]$.

The above representations give the following (AII.3)-(AII.6).

(AII.3) $q^{(N)}(t,[\sigma],[\tau]) \rightarrow q(t, \sigma, \tau)$ uniformly in $(t, \sigma, \tau)$ on each compact subset of $(0, \infty) \times[0,1] \times[0,1]$ as $N \rightarrow \infty$.

(AII.4) $\quad \int_{0}^{1} f([\tau]) q^{(N)}(t,[\sigma],[\tau]) d \tau \rightarrow \int_{0}^{1} f(\tau) q(t, \sigma, \tau) d \tau$ uniformly in $(t, \sigma) \in[0, T]$ $\times[0,1]$ as $N \rightarrow \infty$ for each $f \in C([0,1])$ and $T<\infty$.

(AII.5) There exists a positive constant $C=C(T)$ such that

$$
q^{(N)}\left(t, \frac{k}{N}, \frac{\ell}{N}\right) \leqq C t^{-1 / 2}, \quad t \in(0, T] .
$$

(AII.6) There exists a positive constant $C$ such that

$$
\begin{gathered}
\left|\int_{0}^{t}\left\{q^{(N)}\left(s, \frac{k}{N}, \frac{\ell_{1}}{N}\right)-q^{(N)}\left(s, \frac{k}{N}, \frac{\ell_{2}}{N}\right)\right\} d s\right| \\
\leqq C\left(\frac{\ell_{1}-\ell_{2}}{N}\right)^{1 / 2}, \quad t \in[0, \infty) .
\end{gathered}
$$

(b) We often denote $q(t, \sigma, \tau)$ by $q_{i}(t, \sigma, \tau)(i=1,2,3)$ to clarify the boundary conditions (2.9) of the operator (1/2) $\Delta$ in the cases (I), (II) and (III), respectively.

The following four estimates on $q$ are well-known.

(AII.7) For each $T<\infty$, there exists a positive constant $C$ such that

$$
0 \leqq q(t, \sigma, \tau) \leqq C t^{-1 / 2} \quad \text { for }(t, \sigma, \tau) \in(0, T] \times[0,1] \times[0,1] .
$$

(AII.8) For each $0<T<\infty$, there exist positive constants $C$ and $\beta$ such that

$$
\begin{aligned}
& \left|q_{i}(t, \sigma, \tau)-\delta_{i, 1}\right| \leqq C e^{-\beta t} \\
& \quad \text { for }(t, \sigma, \tau) \in[T, \infty) \times[0,1] \times[0,1], \quad i=1,2,3,
\end{aligned}
$$

where $\delta_{i, j}$ is the Kronecker's $\delta$.

(AII.9) $\quad \int_{0}^{1} q(t, \sigma, \tau) d \tau \leqq 1, \quad(t, \sigma) \in(0, \infty) \times[0,1]$.

(AII.10) For each $0<T_{1}, T_{2}<\infty$,

$$
\sup _{(t, \sigma, \tau) \in\left[T_{1}, T_{2}\right] \times[0,1] \times[0,1]}\left|\frac{\partial^{2} q_{3}}{\partial t \partial \sigma}(t, \sigma, \tau)\right|<\infty .
$$


These estimates imply the followings immediately; there exists a positive constant $C_{1}$ such that

(AII.11) $\quad \int_{0}^{1}\left|q_{i}(t, \sigma, \tau)-\delta_{i, 1}\right| d \tau \leqq C_{1} e^{-\beta t} \quad$ for $(t, \sigma) \in(0, \infty) \times[0,1]$,

and

(AII.12)

$$
\begin{aligned}
q_{i}(t, \sigma, \tau) \leqq & C_{1}\left(t^{-1 / 2}+\delta_{i, 1}\right) \\
& \quad \text { for }(t, \sigma, \tau) \in(0, \infty) \times[0,1] \times[0,1], \quad i=1,2,3 .
\end{aligned}
$$

For each $i=1,2,3$, we set

$$
G_{i}(t ; \sigma, \tau)=\int_{0}^{t}\left\{q_{i}(s, \sigma, \tau)-\delta_{i, 1}\right\} d s
$$

and

$$
G_{i}(\sigma, \tau)=G_{i}(\infty ; \sigma, \tau) .
$$

Since $G_{i}(\sigma, \tau)(i=1,2,3)$ are the Green functions of $(1 / 2) \Delta$ with the boundary conditions (2.9), they are given as follows.

$$
\begin{aligned}
& G_{1}(\sigma, \tau)=2\left\{\sigma \wedge \tau-\left(\sigma-\sigma^{2} / 2\right)-\left(\tau-\tau^{2} / 2\right)+1 / 3\right\} \\
& G_{2}(\sigma, \tau)=2(\sigma \wedge \tau) \\
& G_{3}(\sigma, \tau)=2(\sigma \wedge \tau)\{1-(\sigma \vee \tau)\},
\end{aligned}
$$

where $\sigma \wedge \tau=\min (\sigma, \tau)$ and $\sigma \vee \tau=\max (\sigma, \tau)$. These equalities give that (AII.13)

$$
G_{i}(\sigma, \sigma)+G_{i}(\tau, \tau)-2 G_{i}(\sigma, \tau)=2|\sigma-\tau|\left\{1-\delta_{i, 3}|\sigma-\tau|\right\}
$$

and

(AII.14) $\left|G\left(\sigma, \tau_{1}\right)-G\left(\sigma, \tau_{2}\right)\right| \leqq 2\left|\tau_{1}-\tau_{2}\right|$.

Since the Chapman-Kolmogorov equation of $q$ implies

$$
G(t ; \sigma, \tau)=G(\sigma, \tau)-\int_{0}^{1} G\left(\tau^{\prime}, \tau\right) q_{i}\left(t, \sigma, \tau^{\prime}\right) d \tau^{\prime}
$$

we have

(AII.15)

$$
|G(t ; \sigma, \sigma)+G(t ; \tau, \tau)-2 G(t ; \sigma, \tau)| \leqq 6|\sigma-\tau|
$$

\section{Appendix III}

We consider the following two equations.

(AIII.1) $\quad d X_{t}(\sigma)=d B_{t}(\sigma)+b\left(X_{t}(\sigma)\right) d t+\frac{1}{2} \Delta X_{t}(\sigma) d t, \quad \sigma \in[0,1], \quad t>0$, 
(AIII.2) $\left\{\begin{array}{c}d X_{t}(\sigma)=V_{t}(\sigma) d t \\ d V_{t}(\sigma)=\beta d B_{t}(\sigma)+\beta b\left(X_{t}(\sigma)\right) d t+\frac{\beta}{2} \Delta X_{t}(\sigma) d t-\beta V_{t}(\sigma) d t \\ \sigma \in[0,1], \quad t>0, \quad \beta \geqq 1 .\end{array}\right.$

In the above two equations, $B_{t}(\sigma)$ is a cylindrical Brownian motion on $L^{2}\left([0,1], R^{d}\right)$ and $b(x)$ is assumed to be an $R^{d}$-valued Lipschitz continuous function defined on $R^{d}$. When $b(x)=-\nabla U(x) / 2$ with a potential function $U(x)$, the equation (AIII.1) was discussed in $\S 4$, while the equation (AIII.2) may be thought to represent the Ornstein-Uhlenbeck theory for the string with the friction intensity $\beta$.

Here, we show first an existence and uniqueness theorem of the solution of the equation (AIII.2) and secondly show that the solution $X_{t}(\sigma)=$ $X_{t}^{(\beta)}(\sigma)$ of (AIII.2) tends to that of (AIII.1) as $\beta \rightarrow \infty$. For simplicity, we discuss only in the cases (II) and (III) assuming $A_{0}=A_{1}=0$.

The operator $-\Delta$ on the space $L^{2}\left([0,1], R^{d}\right)$ has pure point spectrum $\left\{\lambda_{i}\right\}_{i=1}^{\infty}$. Let $\phi_{i}$ be the normalized eigenvector corresponding to the $\lambda_{i}$. Setting $x_{i}(t)=\left\langle X_{t}(\sigma), \phi_{i}(\sigma)\right\rangle$ and $v_{i}(t)=\left\langle V_{t}(\sigma), \phi_{i}(\sigma)\right\rangle$, the equation (AIII.2) turns into the following system.

(AIII.3)

$$
\left\{\begin{array}{l}
d x_{i}(t)=v_{i}(t) d t \\
d v_{i}(t)=\beta d w_{i}(t)+\beta b_{i}\left(x_{t}\right) d t-\frac{\beta}{2} \lambda_{i} x_{i}(t) d t-\beta v_{i}(t) d t \\
i=1,2, \cdots
\end{array}\right.
$$

where

$$
x_{t}=\left(x_{i}(t)\right)_{i=1}^{\infty}, \quad w_{i}(t)=\left\langle B_{t}(\sigma), \phi_{i}(\sigma)\right\rangle
$$

and

$$
b_{i}(x)=\left\langle b\left(\sum_{k=1}^{\infty} x_{k} \phi_{k}(\sigma)\right), \phi_{i}(\sigma)\right\rangle \quad \text { for } x=\left(x_{k}\right)_{k=1}^{\infty} .
$$

We introduce the following Hilbert space for each $\alpha \in R^{1}$ :

$$
H_{\alpha}=\left\{v=\left(v_{i}\right)_{i=1}^{\infty} \in R^{N} ;\|v\|_{\alpha}^{2}=\sum_{i=1}^{\infty} \lambda_{i}^{\alpha} v_{i}^{2}<\infty\right\} .
$$

We denote by $\pi_{m}^{n}(1 \leqq m \leqq n \leqq \infty)$ the orthogonal projection of $H_{\alpha}$ into the subspace $\left\{v=\left(v_{i}\right)_{i=1}^{\infty} \in H_{\alpha} ; v_{i}=0\right.$ if $i<m$ or $\left.i>n\right\}$. We also denote $\pi_{1}^{n}$ and $\pi_{m+1}^{\infty}$ simply by $\pi^{n}$ and $\pi_{m}$, respectively. Then, we have the following.

Proposition AIII.1. For every initial state $\left(x_{0}, v_{0}\right) \in H_{0} \times H_{-1}$, there 
exists a unique solution $\left(x_{t}, v_{t}\right)$ of (AIII.3) such that $\sup _{t \in[0, T]} E\left[\left\|x_{t}\right\|_{0}^{2}+\left\|v_{t}\right\|_{-1}^{2}\right]$ $<\infty$ for each $T<\infty$ and $\left(x_{t}, v_{t}\right) \in C\left([0, \infty), H_{0} \times H_{-1}\right)$ (a.s.).

Proof. We use the Galerkin method to construct the solution. For given initial state $\left(x_{0}, v_{0}\right)=\left(\left\{x_{i}(0)\right\}_{i=1}^{\infty},\left\{v_{i}(0)\right\}_{i=1}^{\infty}\right)$, we determine the processes $\left(x_{t}^{(n)}, v_{t}^{(n)}\right)=\left(\left\{x_{i}^{(n)}(t)\right\}_{i=1}^{\infty},\left\{v_{i}^{(n)}(t)\right\}_{i=1}^{\infty}\right) \in H_{0} \times H_{-1}$ by solving the following sequence of finite dimensional stochastic differential equations $(n \in N)$ :

(AIII.4)

$$
\left\{\begin{array}{l}
d x_{i}^{(n)}(t)=v_{i}^{(n)}(t) d t \\
d v_{i}^{(n)}(t)=\beta d w_{i}(t)+\beta b_{i}\left(x_{t}^{(n)}\right) d t-\frac{\beta}{2} \lambda_{i} x_{i}^{(n)}(t) d t-\beta v_{i}^{(n)}(t) d t \\
x_{i}^{(n)}(0)=x_{i}(0), \quad v_{i}^{(n)}(0)=v_{i}(0), \quad 1 \leqq i \leqq n, \\
x_{j}^{(n)}(t)=x_{j}(0), \quad v_{j}^{(n)}(t)=v_{j}(0), \quad j \geqq n+1, \quad t \in[0, T] .
\end{array}\right.
$$

To prove the convergence of the sequence $\left\{\left(x_{t}^{(n)}, v_{t}^{(n)}\right)\right\}_{n=1}^{\infty}$, we set, for $m<n$,

$$
\begin{aligned}
& I(t, m, n)=E\left[\sup _{0 \leqq s \leqq t}\left\{\frac{\beta}{2}\left\|x_{s}^{(n)}-x_{s}^{(m)}\right\|_{0}^{2}+\left\|v_{s}^{(n)}-v_{s}^{(m)}\right\|_{-1}^{2}\right\}\right], \\
& J^{1}(t, m, n)=E\left[\sup _{0 \leqq s \leqq t}\left\{\frac{\beta}{2}\left\|\pi^{m}\left(x_{s}^{(n)}-x_{s}^{(m)}\right)\right\|_{0}^{2}+\left\|\pi^{m}\left(v_{s}^{(n)}-v_{s}^{(m)}\right)\right\|_{-1}^{2}\right\}\right]
\end{aligned}
$$

and

$$
J^{2}(t, m, n)=E\left[\sup _{0 \leqq s \leqq t}\left\{\frac{\beta}{2}\left\|\pi_{m} x_{s}^{(n)}\right\|_{0_{-}}^{2\urcorner}+\left\|\pi_{m} v_{s}^{(n)}\right\|_{-1}^{2}\right\}\right]
$$

By using Itô's formula, we have

$$
\begin{aligned}
& d\left[\frac{\beta}{2} \lambda_{i}\left(x_{i}^{(n)}(t)-x_{i}^{(m)}(t)\right)^{2}+\left(v_{i}^{(n)}(t)-v_{i}^{(m)}(t)\right)^{2}\right] \\
& \quad=2\left(v_{i}^{(n)}(t)-v_{i}^{(m)}(t)\right)\left\{\beta\left(b_{i}\left(x_{t}^{(n)}\right)-b_{i}\left(x_{t}^{(m)}\right)\right) d t-\beta\left(v_{i}^{(n)}(t)-v_{i}^{(m)}(t)\right) d t\right\} \\
& \quad \leqq \frac{\beta}{2}\left\{b_{i}\left(x_{t}^{(n)}\right)-b_{i}\left(x_{t}^{(m)}\right)\right\}^{2} d t \\
& \quad \leqq \beta K\left\|x_{t}^{(n)}-x_{t}^{(m)}\right\|_{0}^{2} d t, \quad \text { for } i \leqq m,
\end{aligned}
$$

which imply that

$$
\begin{aligned}
J^{1}(t, m, n) & \leqq K \sum_{i=1}^{m} \lambda_{i}^{-1} \int_{0}^{t} \beta E\left[\left\|x_{s}^{(n)}-x_{s}^{(m)}\right\|_{0}^{2}\right] d s \\
& \leqq 2 K \sum_{i=1}^{\infty} \lambda_{i}^{-1} \int_{0}^{t} I(s, m, n) d s .
\end{aligned}
$$

Again by using Itô's formula, we have 


$$
\begin{aligned}
& d\left[\frac{\beta}{2} \lambda_{i}\left(x_{i}^{(n)}(t)\right)^{2}+\left(v_{i}^{(n)}(t)\right)^{2}\right] \\
& \quad=2 v_{i}^{(n)}(t)\left\{\beta d w_{i}(t)+\beta b_{i}\left(x_{t}^{(n)}\right) d t-\beta v_{i}^{(n)}(t) d t\right\}+\beta^{2} d t \\
& \quad \leqq 2 \beta v_{i}^{(n)}(t) d w_{i}(t)+\frac{\beta}{2} b_{i}^{2}\left(x_{t}^{(n)}\right) d t+\beta^{2} d t \quad \text { for } i \leqq n
\end{aligned}
$$

which prove that

$$
\begin{aligned}
& J^{2}(t, m, n) \leqq \frac{\beta}{2}\left\|\pi_{m} x_{0}\right\|_{0}^{2}+\left\|\pi_{m} v_{0}\right\|_{-1}^{2} \\
& \left.\quad+E\left[\sup _{0 \leqq s \leqq t} \sum_{i=m+1}^{n} \lambda_{i}^{-1} \int_{0}^{s} 2 \beta v_{i}^{(n)}(u) d w_{i}(u)\right]\right] \\
& +E\left[\sup _{0 \leqq s \leqq t} \sum_{i=m+1}^{n} \lambda_{i}^{-1}\left\{\int_{0}^{s} \frac{\beta}{2} b_{i}^{2}\left(x_{u}^{(n)}\right) d u+\beta^{2} s\right\}\right] .
\end{aligned}
$$

The third term in the right hand side of (AIII.6) is bounded by

$$
\begin{gathered}
E\left[\sup _{0 \leqq s \leqq t} \sum_{i=m+1}^{n}\left\{\beta^{2} \lambda_{i}^{-1}+\lambda_{i}^{-1}\left(\int_{0}^{s} v_{i}^{(n)}(u) d w_{i}(u)\right)^{2}\right\}\right] \\
\leqq \beta^{2} \sum_{i=m+1}^{n} \lambda_{i}^{-1}+\sum_{i=m+1}^{n} 4 \lambda_{i}^{-1} \int_{0}^{t} E\left[\left(v_{i}^{(n)}(s)\right)^{2}\right] d s .
\end{gathered}
$$

While, noting that

$$
\sum_{i=1}^{\infty} b_{i}^{2}(x) \leqq K\left(1+\|x\|_{0}^{2}\right)
$$

the fourth term is bounded by

$$
\text { (AIII.8) } \quad \sum_{i=m+1}^{n} \lambda_{i}^{-1}\left\{\int_{0}^{t} \frac{\beta}{2} K\left(1+E\left[\left\|x_{s}^{(n)}\right\|_{0}^{2}\right]\right) d s+\beta^{2} t\right\} \text {. }
$$

Summing up the estimates (AIII.6-8), we have

$$
\begin{aligned}
J^{2}(t, m, n) & \leqq C_{1}(m, \beta)+C_{2}(m) \beta \int_{0}^{t} E\left[\left\|x_{s}^{(n)}\right\|_{0}^{2}\right] d s+4 \int_{0}^{t} E\left[\left\|\pi_{m+1}^{n} v_{s}^{(n)}\right\|_{-1}^{2}\right] d s \\
& \leqq C_{1}(m, \beta)+C_{2}(m) \beta \int_{0}^{t} E\left[\left\|x_{s}^{(n)}\right\|_{0}^{2}\right] d s+4 \int_{0}^{t} J^{2}(s, m, n) d s, \quad t \leqq T,
\end{aligned}
$$

where

$$
\begin{aligned}
& C_{1}(m, \beta)=\frac{\beta}{2}\left\|\pi_{m} x_{0}\right\|_{0}^{2}+\left\|\pi_{m} v_{0}\right\|_{-1}^{2}+\left(\beta^{2}+\frac{\beta}{2} K T+\beta^{2} T\right) \sum_{i=m+1}^{\infty} \lambda_{i}^{-1}, \\
& C_{2}(m)=\frac{1}{2} K \sum_{i=m+1}^{\infty} \lambda_{i}^{-1} .
\end{aligned}
$$

This implies that 
(AIII.9) $\quad J^{2}(t, m, n) \leqq\left\{C_{1}(m, \beta)+C_{2}(m) \beta \int_{0}^{t} E\left[\left\|x_{s}^{(n)}\right\|_{0}^{2}\right] d s\right\} e^{4 T}, \quad t \leqq T$

In particular, by taking $m=0$, we get

$$
J^{2}(t, 0, n) \leqq e^{4 T}\left\{C_{1}(0, \beta)+2 C_{2}(0) \int_{0}^{t} J^{2}(s, 0, n) d s\right\},
$$

which proves that

$$
J^{2}(t, 0, n) \leqq e^{4 T} C_{1}(0, \beta) e^{2 C_{2}(0) e^{4 T} T} \equiv C_{3}(\beta) .
$$

Substituting (AIII.10) into the right hand side of (AIII.9), we have

(AIII.11) $\quad J^{2}(t, m, n) \leqq\left\{C_{1}(m, \beta)+2 C_{2}(m) C_{3}(\beta) T\right\} e^{4 T} \equiv C_{4}(m, \beta)$.

Since $I(t, m, n)$ is bounded by

$$
J^{1}(t, m, n)+2\left\{J^{2}(t, m, n)+\frac{\beta}{2}\left\|\pi_{m} x_{0}\right\|_{0}^{2}+\left\|\pi_{m} v_{0}\right\|_{-1}^{2}\right\},
$$

combining (AIII.11) with (AIII.5), we have

$$
I(t, m, n) \leqq 2 C_{1}(m, \beta)+2 C_{4}(m, \beta)+4 C_{2}(0) \int_{0}^{t} I(s, m, n) d s, \quad \text { for } t \leqq T,
$$

and this implies that

$$
I(t, m, n) \leqq\left\{2 C_{1}(m, \beta)+2 C_{4}(m, \beta)\right\} e^{4 C_{2}(0) T}, \quad t \leqq T .
$$

We therefore have

$$
\lim _{\substack{n>m \\ m \rightarrow \infty}} E\left[\sup _{0 \leqq t \leqq T}\left\{\frac{\beta}{2}\left\|x_{t}^{(n)}-x_{t}^{(m)}\right\|_{0}^{2}+\left\|v_{t}^{(n)}-v_{t}^{(m)}\right\|_{-1}^{2}\right\}\right]=0 .
$$

This proves that there exists an $H_{0} \times H_{-1}$-valued continuous process $\left(x_{t}, v_{t}\right)$ such that

$$
\lim _{n \rightarrow \infty} E\left[\sup _{0 \leqq t \leqq T}\left\{\frac{\beta}{2}\left\|x_{t}^{(n)}-x_{t}\right\|_{0}^{2}+\left\|v_{t}^{(n)}-v_{t}\right\|_{-1}^{2}\right\}\right]=0
$$

We can easily show that $\left(x_{t}, v_{t}\right)$ is the unique solution of the equation (AIII.3).

(Q.E.D.)

The solution $\left(x_{t}, v_{t}\right)=\left(\left\{x_{i}(t)\right\}_{i=1}^{\infty},\left\{v_{i}(t)\right\}_{i=1}^{\infty}\right)$ of the equation (AIII.3) depends on the parameter $\beta$, so that we denote the solution by $\left(x_{t}^{(\beta)}, v_{t}^{(\beta)}\right)=\left(\left\{x_{i}^{(\beta)}(t)\right\}_{i=1}^{\infty}\right.$, $\left.\left\{v_{i}^{(\beta)}(t)\right\}_{i=1}^{\infty}\right)$. Setting $x_{i}(t)=\left\langle X_{t}(\sigma), \phi_{i}(\sigma)\right\rangle$ again, the equation (AIII.1) becomes the following equation for $x_{t}=\left(x_{i}(t)\right)_{i=1}^{\infty} \in C([0, \infty), H)$ (a.s.). 
(AIII.12) $\quad d x_{i}(t)=d w_{i}(t)+b_{i}\left(x_{t}\right) d t-\frac{1}{2} \lambda_{i} x_{i}(t) d t, \quad i=1,2, \cdots$

Assuming that the function $b$ is bounded, we show that the $x_{t}^{(\beta)}$ tends to the $x_{t}$ as $\beta \rightarrow \infty$.

Proposition AIII.2. For every $x(0)$ and $v(0) \in H_{0}$, the solution $x_{t}^{(\beta)}$ of (AIII.3) with the initial condition $\left(x_{0}^{(\beta)}, v_{0}^{(\beta)}\right)=(x(0), v(0))$ converges to the solution $x_{t}$ of (AIII.12) with the initial condition $x_{0}=x(0)$ as $\beta \rightarrow \infty$ in the following sense. For every $\varepsilon: 0<\varepsilon<1 / d$,

$$
\lim _{\beta\left(\in A_{s}\right) \rightarrow \infty} E\left[\left\|x_{t}^{(\beta)}-x_{t}\right\|_{0}^{2}\right]=0
$$

uniformly in $t$ on each compact set of $(0, \infty)$, where the set $\Lambda_{s}$ is given by

$$
\Lambda_{\mathrm{s}}=\left\{\beta>0 ;\left|\beta-2 \lambda_{i}\right|>\varepsilon \sqrt{\beta} \text { for every } i=1,2, \cdots\right\} .
$$

Proof. We state only the outline. The equation (AIII.3) can be rewritten in the following integral equation.

$$
\begin{aligned}
x_{i}^{(\beta)}(t)= & \left(a_{+}-a_{-}\right)^{-1}\left\{\left(a_{+} e^{a_{-} t}-a_{-} e^{a_{+} t}\right) x_{i}(0)+\left(e^{a_{+} t}-e^{a_{-} t}\right) v_{i}(0)\right\} \\
& +\beta\left(a_{+}-a_{-}\right)^{-1} \int_{0}^{t}\left(e^{a_{+}(t-s)}-e^{a_{-}(t-s)}\right) d w_{i}(s) \\
& +\beta\left(a_{+}-a_{-}\right)^{-1} \int_{0}^{t}\left(e^{a_{+}(t-s)}-e^{a_{-}(t-s)}\right) b_{i}\left(x_{s}^{(\beta)}\right) d s \\
\equiv & x_{i, 1}^{(\beta)}(t)+x_{i, 2}^{(\beta)}(t)+x_{i, 3}^{(\beta)}(t), \quad i=1,2, \cdots, \quad \beta \neq 2 \lambda_{i}
\end{aligned}
$$

where

$$
a_{ \pm}=a_{ \pm}(i, \beta)=\frac{1}{2}\left(-\beta \pm \sqrt{\beta^{2}-2 \beta \lambda_{i}}\right) .
$$

While, the equation (AIII.12) is equivalent to the following.

$$
\begin{aligned}
x_{i}(t) & =e^{-\lambda_{i} t / 2} x_{i}(0)+\int_{0}^{t} e^{-\lambda_{i}(t-s) / 2} d w_{i}(s)+\int_{0}^{t} e^{-\lambda_{i}(t-s) / 2} b_{i}\left(x_{s}\right) d s \\
& \equiv x_{i, 1}(t)+x_{i, 2}(t)+x_{i, 3}(t), \quad i=1,2, \cdots
\end{aligned}
$$

By estimating three terms

$$
\sum_{i=1}^{\infty} E\left[\left|x_{i, j}^{(\beta)}(t)-x_{i, j}(t)\right|^{2}\right], \quad j=1,2,3
$$

independently, we finally arrive at the following estimate. 


$$
\begin{aligned}
E\left[\left\|x_{t}^{(\beta)}-x_{t}\right\|_{0}^{2}\right] \leqq & f(\beta)+C_{1}\left\{\beta^{1 / 2} \exp \left(-\beta^{2 / 3} t\right)+\beta^{1 / 16} \exp \left(-\beta^{1 / 4} t\right)\right\} \\
& +C_{1} \int_{0}^{t} E\left[\left\|x_{s}^{(\beta)}-x_{s}\right\|_{0}^{2}\right] d s, \quad t \leqq T, \quad \beta \in \Lambda_{\varepsilon},
\end{aligned}
$$

with $f(\beta)=f\left(\beta ; T,\|x(0)\|_{0},\|v(0)\|_{0}\right)$ and $C_{1}=C_{1}\left(T,\|x(0)\|_{0},\|v(0)\|_{0}\right)$, where the function $f(\beta)$ satisfies that

$$
f(\beta) \longrightarrow 0 \text { as } \beta\left(\in \Lambda_{\varepsilon}\right) \longrightarrow \infty .
$$

Therefore, we have

$$
\begin{aligned}
& E\left[\left\|x_{t}^{(\beta)}-x_{t}\right\|_{0}^{2}\right] \leqq f(\beta)+C_{1}\left\{\beta^{1 / 2} \exp \left(-\beta^{2 / 3} t\right)+\beta^{1 / 16} \exp \left(-\beta^{1 / 4} t\right)\right\} \\
& \quad+\int_{0}^{t} e^{C_{1}(t-s)}\left\{f(\beta)+C_{1}\left(\beta^{1 / 2} \exp \left(-\beta^{2 / 3} s\right)+\beta^{1 / 16} \exp \left(-\beta^{1 / 4} s\right)\right)\right\} d s \\
& \leqq C_{1}\left\{\beta^{1 / 2} \exp \left(-\beta^{2 / 3} t\right)+\beta^{1 / 16} \exp \left(-\beta^{1 / 4} t\right)\right\}+C_{2}\left(f(\beta)+\beta^{-1 / 6}\right), \\
& t \leqq T .
\end{aligned}
$$

This implies the desired conclusion.

(Q.E.D.)

Remark AIII.1. (i) E. Cabaña [4] discussed the Ornstein-Uhlenbeck theory for the string mathematically, however, the equation of the string studied by him was linear.

(ii) In E. Nelson's book [18], one can find the proof to show that, in finite dimensional case, the Einstein-Smoluchowski theory is given by the limit of Ornstein-Uhlenbeck theory.

\section{REFERENCES}

[1] S. Agmon, Lectures on elliptic boundary value problems, Princeton, N. J.: D. Van Nostrand Co., Inc., 1965.

[2] R. Arima, On general boundary value problem for parabolic equations, J. Math. Kyoto Univ., 4 (1964), 207-243.

[ 3 ] Yu. N. Blagovescenskii and M. I. Freidlin, Certain properties of diffusion processes depending on a parameter, Soviet Math. Dokl., 2 (1961), 633-636.

[4: ] E. Cabaña, The vibrating string forced by white noise, Z. Wahrsch. Verw. Gebiete, 15 (1970), 111-130.

[5] D. A. Dawson, Stochastic evolution equations, Math. Biosci., 15 (1972), 287-316.

[ 6 ] —-, Stochastic evolution equations and related measure processes, J. Multivariate Anal., 5 (1975), 1-55.

[ 7 ] H. Doss et G. Royer, Processus de diffusion associe aux mesures de Gibbs sur $\boldsymbol{R}^{z^{d}}$, Z. Wahrsch. Verw. Gebiete, 46 (1978), 107-124.

[ 8 ] I. I. Gihman and A. V. Skorohod, Stochastic differential equation, Springer-Verlag, Berlin Heidelberg New York, 1972.

[ 9 ] R. A. Holley and D. W. Stroock, Generalized Ornstein-Uhlenbeck processes and infinite particle branching Brownian motions, Publ. RIMS Kyoto Univ., 14 (1978), 741-788.

[10] K. Itô, Stochastic analysis in infinite dimensions, Stochastic Analysis (ed. by A. Friedman and M. Pinsky), Academic Press (1978), 187-197. 
[11] A. N. Kolmogorov, Zur Umkerbarkeit der statistischen Naturgesetze, Math. Ann., 113 (1937), 766-772.

[12] H.-H. Kuo, Stochastic integrals in abstract Wiener space (II) : regularity properties, Nagoya Math. J., 50 (1973), 89-116.

[13] R. Lang, Unendlich-dimensionale Wienerprozesse mit Wechselwirkung I, II, Z. Wahrsch. Verw. Gebiete, 38 (1977), 55-72 and 39 (1977), 277-299.

[14] J. L. Lions and E. Magenes, Non-homogeneous boundary value problems and applications I, II, Springer-Verlag Berlin Heidelberg New York, 1972.

[15] R. Marcus, Parabolic Itô equations, Trans. Amer. Math. Soc., 198 (1974), 177-190.

[16] H. P. McKean Jr., Stochastic integrals, Academic Press, New York London, 1969.

[17] S. A. Molchanov, Diffusion processes and Riemannian geometry, Russian Math. Surveys, 30 (1975), 1-63.

[18] E. Nelson, Dynamical theories of Brownian motion, Princeton, N. J., Princeton Univ. Press, 1967.

[19] M. Yor, Existence et unicité de diffusions à valuers dans un espace de Hilbert, Ann. Inst. H. Poincaré, 10 (1974), 55-88.

Department of Mathematics

Faculty of Science

Nagoya University

Chikusa-ku, Nagoya 464

Japan 